Molecular Control of Electron and Hole Transfer Processes: Theory and Applications

\author{
Marshall D. Newton ${ }^{*}, \mathrm{PhD}$ \\ Senior Chemist, Brookhaven National Laboratory \\ Adjunct Professor of Chemistry, State University of New York at Stony Brook

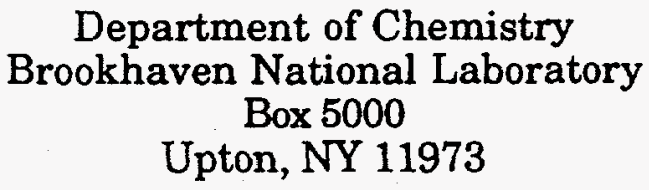 \\ 516-282-4366 (phone) \\ 516-282-5815 (fax) \\ newton1@bnl.gov
}

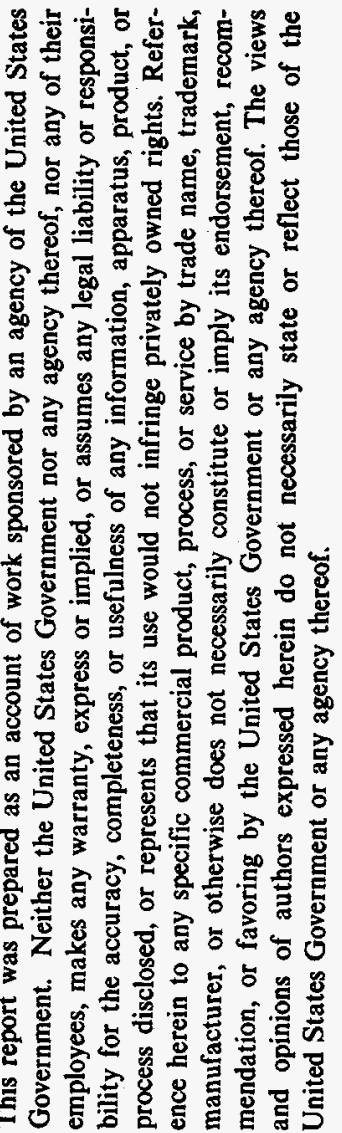

Robert J. Cave, $\mathrm{PhD}$

Associate Professor, Harvey Mudd College

Department of Chemistry

Harvey Mudd College

Claremont, CA 91711

$909-607-3504$

$909-621-8465$

cave@thuban.ac.hmc.edu

${ }^{*}$ Contact for queries

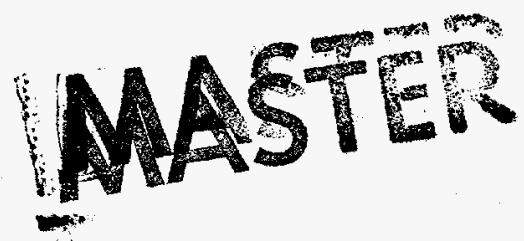

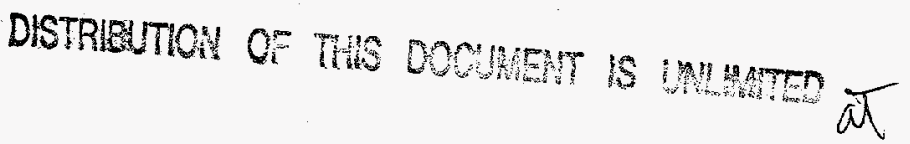


$y$ . 
1 Introduction (p1)

2 Theoretical Foundation: Preliminaries (p3)

2.1 Kinetic Context (p3)

2.2 What particles (and how many) are actually transferred in "electron transfer" processes? (p7)

2.3 The Nature of "Electron Tunneling". (p8)

$2.4 \quad$ Influence of Medium on $T_{\text {if }}$ (p10)

3 Theoretical Models for $\mathbf{T}_{\text {if }}$ in the Two-State Approximation (TSA) (p11)

3.1 Superexchange via Perturbation Theory (p14)

3.2 Variational Models. (p20)

3.3 Extensions and Embellishments of the McConnell Model. (p21)

3.4 Many-particle Perspectives. (p23)

4. More General Models for $\mathbf{T}_{\text {if. }}$

4.1 The Mulliken-Hush Model (p30)

4.2 Generalized Mulliken-Hush Model.(GMH) (p33)

5 Computational Applications (p35)

5.1 Orbital Models (p37)

5.2 Trends in $T_{\text {if }}$ for Saturated Bridges. (p39) 
5.3 Analysis of Superexchange Coupling in Terms of Natural Bond Orbitals (NBO's). (p44)

5.4 Applications of the GMH (p48)

6. Concluding Remarks (p53)

7. Acknowledgment (p54) 


\title{
Molecular Control of Electron and Hole Transfer Processes: Theory and Applications
}

\author{
M. D. Newtona and R. J. Caveb \\ a Department of Chemistry, Brookhaven National Laboratory, Upton, New York, 11973-5000; \\ ${ }^{b}$ Department of Chemistry, Harvey Mudd College, Claremont, CA 91711
}

\section{Introduction}

Recent decades have seen remarkable advances in microscopic understanding of electron transfer $(e t)$ processes in widely ranging contexts, including solid-state, liquid solution, and complex biological assemblies [1-6]. This understanding is reflected in theoretical models of rapidly increasing sophistication [7-14], which relate the dynamical and kinetic behavior of et processes to the underlying structural, energetic and electronic properties of the reactive systems. Typically, one identifies, and treats quantum mechanically, local molecular donor (D) and acceptor (A) sites, and then formulates the manner in which the effective coupling facilitating the et process is mediated by the energetic and electronic features of the intervening medium (the "bridge" (B)) as well as the surrounding environment (fig 1). The energetics and dynamics associated with activation are treated with either classical or quantum mechanical models. The theoretical models play an important dual role, on the one hand leading (in conjunction with modern computing power) to realistic computational implementation, and on the other, allowing analysis of the results of such calculations (as well as those from experiment) in terms of compact predictive models grounded in simple concepts of chemical structure and bonding. The power of the current armament of theoretical tools for confronting the challenges posed by et dynamics is 
underscored by their generic applicability: e.g., to thermal, optical and photoinitiated processes, both homogeneous and interfacial (e.g., at electrodes).

The continuing challenge, of course, is to convert the rapidly accumulating mechanistic information about et kinetics (often representable in terms of simple rate constants) into precise tools for fine-tuned control of the kinetics and design of molecular-based systems which meet specified et characteristics. Progress toward these latter objectives is yielding increasingly productive contact with the world of microelectronic devices --- i.e., molecular electronics [15-16], the guiding focus of the current volume. For some time, the literature has offered inspiring examples of the fruitful application of orbital or other quantum chemical concepts in formulating idealized models for devices such as rectifiers, switches and registers, and in general articulating the concept of "molecular wires" [17-27]. The close relationship between scanning tunneling microscopy (STM) and chemical electron transfer processes have been noted in a number of recent papers $[20,24,28]$. On a more exotic note, one of nature's more successful devices, photosynthesis, has stimulated intensive theoretical and computational studies in recent years [29-32].

With the above background in mind, the primary goal of this chapter is to report recent advances in the modeling, calculation, and analysis of electronic coupling in complex molecular aggregates, thereby allowing an assessment of current progress toward the goal of molecular-level control and design. The control of electron transfer kinetics (i.e., enhancing desired processes, while inhibiting others) involves, of course, system energetics (especially activation and reorganization energies) as well as electronic coupling, which is most directly relevant only after the system has reached the appropriate point (or region) along the "reaction coordinate". Nevertheless, to focus the discussion in this chapter, we will consider such energetics, and the associated molecular and solvent 
coordinates which control then, only to the extent that they bear on the analysis of the electronic coupling.

In the following sections we will first discuss the formulation of basic et models, including the definition of initial and final states, the role of orbitals and 1particle models in a many-electron context, the utility of various effective Hamiltonians, and the role of vibronic $[10,33]$ as well as purely electronic effects. With these theoretical tools in hand, we will then examine very recent applications to complex molecular systems using the techniques of computational quantum chemistry, followed by detailed analysis of the numerical results. We will then conclude with some comments regarding the current "state of the art" and remaining challenges.

\section{Theoretical Foundation: Preliminaries}

Before launching into a detailed analysis of the electronic aspects of long-range donor/acceptor (D/A) coupling, it is appropriate to establish a kinetic context and introduce some concepts and distinctions crucial to the subsequent discussion. We are interested in D/A coupling primarily as a controlling factor in et kinetics, although it also plays a central role in a number of related processes, including photoelectron and electron transmission spectroscopy [34], magnetic exchange $[32,35]$, and energy transfer $[36,37]$.

\section{$2.1 \quad$ Kinetic Context}

A convenient point of departure is provided by the standard non-adiabatic transition-state (TS) rate-constant expression, [2,3]

$$
k_{e t}^{t s}=(2 \pi / \hbar)\left(T_{i f}\right)^{2}(F C W D),
$$


where the "transfer integral" $\mathrm{T}_{\text {if }}$ is the effective electronic Hamiltonian matrix element coupling the initial $\left(\psi_{\mathrm{i}}\right)$ and final $\left(\psi_{\mathrm{f}}\right)$ states, which differ, respectively, by having an electron localized primarily at the $\mathrm{D}$ and $\mathrm{A}$ sites, as illustrated schematically in fig 1. The Franck-Condon-weighted density of states (FCWD) reflects the influence of all of the nuclear (inertial) modes of the system, generally represented in terms of effective normal coordinates $\left(Q_{v_{i}}\right.$ and $Q_{w_{f}}$, respectively, for the initial and final states) and the associated quantum-mechanical FranckCondon factors. For sufficiently high temperature (where $\hbar \omega<<k_{B} T$ for all Q), FCWD takes on a limiting form proportional to the classical Arrhenius activation factor,

$F C W D \propto \exp \left(-E_{a} / k_{B} T\right)$

where the effective activation energy (actually a free energy) is given in terms of the "reorganization energy" $\left(\mathrm{E}_{\mathrm{r}}\right)$ and the free energy change $\left(\Delta G_{0}\right)$ for the process (see fig. 2),

$E_{a}=\left(E_{r}+\Delta G_{\partial}\right)^{2} / 4 E_{r}$

At lower temperatures, where quantal effects become appreciable for the highfrequency modes, eq (1) may be recast as a superposition of vibronic state-to-state processes [10],

$k_{e t}^{t s}=(2 \pi / \hbar) \sum_{v_{i} w_{f}}\left(P_{v_{i}}\right)\left(V_{v_{i} w_{f}}{ }^{2}\left(F C W D^{\prime}\right)_{v_{i} w_{f}}\right.$

where $\mathbf{P}_{\mathbf{v}_{i}}$ is the normalized distribution of initial vibronic states (typically in terms of Boltzmann factors), where the vibronic factor $\left(V_{v_{i}} w_{f}\right)^{2}$ is given by

$\left(V_{v_{i} w_{\rho}}\right)^{2}=\left(T_{i f}\right)^{2}\left(S_{v_{i} w_{f}}\right)^{2}$ 
and $\left(S_{v i w p}\right)^{2}$ is a vibrational Franck-Condon factor (i.e., the square of the corresponding vibrational overlap integral). The implicit relationship between the quantities $(F C W D)_{v_{i} w_{p}}$ and $F C W D$, as obtained from comparison of eqs (1) and (4), is given by

$F C W D=\sum_{v_{i} w_{f}} P_{v_{i}}\left(S_{v_{i} w_{f}}\right)^{2}(F C W D)_{v_{i} w_{f}}^{\prime}$

In eqs (1), (4b), and (5) we have employed the Condon approximation [38], factoring $T_{\text {if }}$ out of the full vibronic matrix element, with the understanding that $T_{\text {if }}$ is to be evaluated for values of the nuclear coordinates pertinent to the configuration or range of configurations of the system in which the primary electronic transition occurs. The validity of the Condon factorization depends, among other things, on the extent to which $T_{\text {if }}$ varies with the coordinates $Q$, a topic which we return to when discussing computational results in Section 5 . The coordinates of interest in this connection include the reaction coordinate (RC, as in fig 2), as well as others such as conformational modes of the DBA system (fig 1). The influence of fluctuations in these coordinates (and hence in the magnitude of $\left.T_{\text {if }}\right)$ on the overall kinetics depends in detail on the relationship between the timescale for such fluctuations in comparison with the timescales of the other dynamical processes $[7,10,39]$.

The non-adiabatic expressions for $k_{e t}$ (eqs (1) and (4a)) will be valid provided that the rate-determining step is the primary "electron hop" (whose probability is controlled by $T_{i f}$ in contrast to possible alternative dynamical bottlenecks associated with the various inertial degrees of freedom $[7,10]$. Within the TS regime at high temperature, the Landau-Zener $(\mathrm{LZ})$ model $[40]$ in the case of a harmonic system (see fig 2) shows that the non-adiabatic limit is valid when the following inequality is obeyed [2]: 
where $\omega_{\text {eff }}$ is the effective frequency associated with vibrational motion along the reaction coordinate. As the coupling $T_{\text {if }}$ increases beyond the regime defined by inequality (6), not only must one depart from the non-adiabatic limit, but the $t s$ framework itself may become invalid as inertial contributions (e.g., solvent dynamics) begin to play a role in the rate-determining process $[7,10]$, leading even to the possibility that a simple rate constant may not be adequate to account for the kinetics [7]. Furthermore, the simple expression for $\mathrm{E}_{\mathrm{a}}$ (eq (3)), based on the harmonic weak-coupling (small $\mathrm{T}_{\text {if }}$ ) limit (fig 2) must be modified to reflect the consequences of avoided crossing on the height and shape of the barrier $[2,3,14]$.

At lower temperature, where nuclear quantal effects may be significant, analogs of the preceding dynamical analysis may be carried out at the vibronic level (i.e., with $\mathrm{V}_{\mathrm{v}_{\mathrm{i}} \mathrm{w}_{\mathrm{f}}}\left(\mathrm{eq}(4 \mathrm{~b})\right.$ replacing $\mathrm{T}_{\mathrm{if}}$ ) and with $\mathrm{E}_{\mathrm{r}}$ limited to the contribution to reorganization energy from the low-frequency modes [10].

Aside from the initial and final states discussed above, additional complications may arise due to the presence of low-lying intermediate states (such as those associated with the intervening bridge (fig 1), and may be treated either in a high temperature or low-temperature framework $[41,42]$.

In fig 3 we illustrate the diversity of et processes of chemical interest. These include

(1) thermally activated et proceeding from the ground electronic state (fig 3a);

(2) optical et (often designated as "intervalence transfer" in the case of binuclear mixed valence transition metal complexes), occurring vertically from the equilibrium configuration of the initial state (fig $3 a$ ); and 
(3) photoinitiated et (following initial photoexcitation, typically to a locally excited non-charge transfer state), involving charge separation (CS), followed by subsequent photochemistry (not shown) occurring in competition with chargerecombination (CR) back to the ground state (fig $3 b$ ).

2.2 What particles (and how many) are actually transferred in "electron transfer" processes?

In a process of the type (fig 1 )

$D B A \longrightarrow D^{+} B A^{\circ}$

a single electron is nominally transferred from $\mathrm{D}$ to $\mathrm{A}$ (analogous processes involving multiple electron transfer are also possible), where the "bridge" $B$ denotes whatever material lies in the region directly between the local $\mathrm{D}$ and $\mathrm{A}$ sites. Analysis of the DA coupling, based on a detailed examination of the electronic manifolds of $B$, however, reveals that in general the process (7) is more properly viewed as a superposition of a number of charge transfer processes ("pathways") occurring in parallel, including transfer of a electron in one direction, and transfer of a "hole" (an electron-deficient site) in the opposite direction, as well as more complex processes involving both electrons and holes $[13,14,43,44]$. It is essential to recognize the physical (as opposed to semantic) basis of these distinctions. Superposed on the net transfer of charge between D and A, one may also expect local response (i.e., polarization relaxation) of the system to the oxidation and reduction occurring, respectively, at $D$ and $A[14,45]$. We note that "system" in this connection may be broadly understood to include the "solvent" as well as the DBA "solute". 
With the foregoing multi-particle perspective in mind, it is of great interest to determine from combined analysis and calculation the extent to which the manyelectron quantity $\mathrm{T}_{\text {if }}$ may be cast as an effective 1-particle (i.e., orbital) matrix element, TDA, where DA in this context denotes the "donor" and "acceptor" orbitals, confined primarily to be the $\mathrm{D}$ and $\mathrm{A}$ sites, respectively. In particular, it has been found in a number of cases [14] that the many-electron (relaxation) effects may be captured by electronic Franck-Condon - type overlap integrals, $S_{i j}^{e l}$, with magnitude $\geq 0.9$ :

$T_{i f}=S_{i f}^{e l} T_{D A}$

where TDA may be viewed as the matrix element of an effective 1-particle transition operator. In addition to "solute" (i.e., DBA) contributions to $S_{\mathrm{if}}^{\text {el }}$, similar effects due to solvent have been reported [9].

It still remains to assess the contributions of electron and hole processes to $T_{D A}$ in various cases of interest, as discussed in sections 3 and 5. For simplicity we shall use the expression "electron transfer" generically in the remainder of the chapter, noting the electron/hole distinction wherever appropriate.

\subsection{The Nature of "Electron Tunneling".}

The $\mathrm{D} / \mathrm{A}$ coupling embodied in $\mathrm{T}_{\text {if }}$ (or $\mathrm{T}_{\mathrm{DA}}$ ) is often described as an effective "electron tunneling" process. To focus the discussion we will consider specifically the dependence of $T_{\text {if }}$ on the D/A separation distance ( $\mathrm{rDA}_{\mathrm{DA}}$ ), and compare the prediction of two quite distinct models, each of which yields exponential decay:

$T_{i f f}\left(r_{D A}\right) \propto \exp (-(\beta / 2) r D A)$ 
(the decay coefficient is defined so that $T_{i f}{ }^{2}$ in eq (1) decays with coefficient $\beta$ ). The phenomenological Gamow (WKB) model [46] is based on tunneling through an electronically homogeneous medium, represented by an effective 1-dimensional rectangular barrier of height $\Delta^{G}$ and width $\mathrm{rDA}_{\mathrm{DA}}$ :

$\beta^{G}=2\left(2 m_{e} \Delta^{G}\right) 1 / 2 / \hbar$

where $m_{e}$ is the mass (actual or effective) of the tunneling electron.

Most modern treatments of bridge-mediated electronic coupling do not involve tunneling in the literal sense of the Gamow approach and employ electronically inhomogeneous superexchange (se) models $[47,48]$ which take detailed account of the electronic structure of the DBA system (fig 1). In implementing these models one has the choice of how to subdivide the bridge into subunits, $B_{j}$. The optimal choice involves various tradeoffs, including chemical transferability (favoring small subunits), compactness of representation when it comes to synthesizing the overall coupling, $\mathrm{T}_{\text {if }}$ (favoring larger subunits), and theoretical considerations such as the applicability of perturbation theory (here the tradeoff is complex, but often favors larger subunits $[49,50])$. As a simple example for illustration, we adopt the McConnell model [47] based on a bridge consisting of a homologous sequence of units, each of which is represented by a single orbital with energy $\Delta$ relative to the common energy of the $D$ and $A$ orbitals, and with nearest-neighbor (NN) bridge orbitals coupled by the "hopping integral" $t$. From this model, for $|t / \Delta| \ll<1$ we obtain

$\beta s e=-2 \ln |t / \Delta| /(\Delta r)$

where $\Delta r$ is the width of one subunit. 
In comparison with the result from the homogenous model (eq(10)), $\beta^{\text {se }}$ reflects the molecular and electronic structure of $B$, not only through the hopping integral $t$, but also $\Delta$ and $\Delta r$ since the values of all the parameters depend on the specific choice of subunit (we also note, in contrast to eq (10), that in eq (11) the electron mass is implicit in $t$ and $\Delta$ ). Another important distinction lies in the role of the gap $\Delta$ in eqs (10) and eq (11). In the latter case, $\Delta$ (for a particular bridge subunit) is in principle (and in some cases in practice) a spectroscopic observable, whereas the phenomenological $\Delta^{G}$ which represents the full bridge in eq (10) is not directly accessible as an observable. Effective $\Delta^{\mathrm{G}}$ values may, of course, be inferred from eq (10) on the basis of experimental $\beta$ values, but attempts to use experimentally determined energy gaps (e.g., from polarography) frequently lead to exaggerated $\beta$ magnitudes [48]. Eq (10) is more usefully applied to the direct or "through-space" $(t s) \mathrm{D} / \mathrm{A}$ coupling when no bridge is present. In this case, $\Delta^{\mathrm{G}}$ may be identified with the relevant ionization potential of $D$ [28].

In section 3 we introduce generalized superexchange models in which bridge units may have several orbitals (both occupied and unoccupied). The consequences of departing from the weak perturbation limit $(|t / \Delta|<<1)$ are also explored, including the approach to the opposite limit, in which intermediate charge transfer states involving the bridge approach resonance with $\mathrm{D}$ and $\mathrm{A}$ levels. The results of detailed quantum mechanical calculations are analyzed (Section $\mathrm{V}$ ) in terms of these models, and the extent to which complex DBA systems display exponential distance dependence is assessed.

\subsection{Influence of Medium on $T_{\text {if }}$}

While medium effects will not be dealt with here in great detail, it is worthwhile summarizing the distinct types of influence which they might exert on $T_{i f}$ 
magnitudes (over and above the modest many-electron Franck-Condon effect noted above). By medium we refer to the environment of the DBA complex (fig 1). Typically, it will be a polar solvent. Solvent molecules may, of course, constitute part of the "bridge" (as, e.g., in the case of solvent-separated ion pairs [51]), which does not necessarily comprise a complete sequence of covalent linkages between D and $\mathrm{A}$.

The influence of a polar medium is exerted primarily by its electrostatic field, either long-range (as representable by a continuum or molecular-level model) or short-range (e.g., via specific hydrogen-bonding). The field (which varies with fluctuations of the medium) can modulate the tails of the $D$ and $A$ orbitals either directly, by controlling the degree of radial localization of the orbitals, or indirectly, by modifying the energy gaps $(\Delta)$ which control superexchange coupling In practice, such effects may be quite small [52]. In addition to these electrostatic effects, the electronic manifold of the medium in the immediate vicinity of the bridge may provide superexchange pathways which substantially affect the overall magnitude of $T_{i f}$ thus in effect serving to expand the size of the bridge. The operational choice of the "bridge" is guided by the objective of including all sites for which direct orbital participation has an appreciable effect on $T_{\text {if. }}$ In some cases of course, local D and A groups may be defined so as to be in contact (as in contact ion pairs), thus eliminating the need for a "bridge" altogether.

We now turn to the task of formulating models for the initial and final states in an et process and the effective operator which couples them in the transfer matrix element $T_{\text {if }}$ (eq (1)). At the outset, we shall confine our attention to the so-called 
two-state approximation (TSA), in which the dynamics of $e t$ is assumed to be adequately accounted for by the two-component space (denoted as the D/A space) spanned by states in which the transferring electron is primarily confined to the $D$ and $A$ sites $[53,54]$. (When one or both of the two states are degenerate or neardegenerate, a somewhat more elaborate "two-level" model must be adopted [14]). In general, the validity of the TSA rests on the requirement that the gap separating the higher energy intermediate et states involving the bridge from those of the D/A space is large relative to the strength of coupling of $\mathrm{D}$ and $\mathrm{A}$ to the bridge. The large gap condition may be relaxed if the latter coupling is small relative to the band width of the intermediate bridge states [53]. While the basis set for representing the D/A space is to a large extent arbitrary, convenience generally dictates the choice of either the appropriate adiabatic states (i.e., which diagonalize the electronic Hamiltonian of the system), denoted $\psi_{1}$ and $\psi_{2}$, or the "diabatic" states $\psi_{\mathrm{i}}$ and $\psi_{\mathrm{f}}$, related to $\psi_{1}$ and $\psi_{2}$ by a unitary transformation,

$\psi_{1}=\cos \theta \psi_{i}+\sin \theta \psi_{f}$

$\psi_{2}=-\sin \theta \psi_{i}+\cos \theta \psi_{f}$,

defined operationally so as to correspond optimally to the actual (non-stationary) states presumed to be involved in the dynamical process underlying the et kinetics.

For et initiated in the ground state, $\psi_{1}$ and $\psi_{2}$ will generally be the two lowest energy adiabatic states, whereas higher energy states will be involved in photoinitiated et (fig 3). We emphasize that the definition of $\psi_{\mathrm{i}}$ and $\psi_{\mathrm{f}}$ is never unique, and the utility of a given prescription must ultimately rest on the results of detailed applications, which we consider below. Clearly, in an et process, $\psi_{i}$ and $\Psi_{\mathrm{f}}$ are designed to correspond to charge-localized valence bond structures. Such structures, aside from their chemically-intuitive appeal, also have the advantage 
that their coupling is dominated by the electronic Hamiltonian, with only minor non-Born Oppenheimer coupling (i.e., from the nuclear momentum operator) expected [2].

In thermally activated $e t$ we are interested in the electronic states at the transition state (TS). When the system is at equilibrium in either the initial or final state (where $\mathrm{D}$ and $\mathrm{A}$ are well out of resonance), the diabatic states can be taken as essentially the same as their adiabatic counterparts. When the system with weakly coupled D and A is suddenly carried into the TS by a fluctuation, we adopt the picture that the system remains in the (now non stationary) $\psi_{i}$ state until it dynamically tunnels to $\psi_{\mathrm{f}}$. In the TS configuration, where D and A are essentially in resonance, the adiabatic states would depart sharply from $\psi_{i}$ and $\psi_{f}$ (due to delocalization associated with the resonant $\mathrm{D}$ and $\mathrm{A}$ sites). However, in the TS kinetic theory, the TS does not last long enough for the possibility of adiabatic (stationary) states to be relevant, and $\psi_{\mathrm{f}}$ is assumed to decay irreversibly to its equilibrium configuration [2]. The required resonance of $\mathrm{D}$ and $\mathrm{A}$ is a statement of the Franck-Condon control of thermally activated et $[2,3,14]$; i.e., at the TS

$\left\langle\psi_{i} H^{e l} \psi_{i}>=\left\langle\psi_{f} H^{e l} \psi_{f}\right\rangle\right.$

and this electronic energy matching will be central to most of the models for $T_{\text {if }}$ given below. Of course, vibronic effects allow some departure from strict electronic energy matching in thermal processes. In optical et, the photon energy balances the electronic mismatch associated with vertical excitation from equilibrium. Here, as noted above, the distinction between adiabatic and diabatic states is likely to be minor (for a possible exception, see [55]) and a different formulation of the effective coupling, $T_{\mathrm{if}}$, is required[14], as discussed in Section 4. 
Returning to the case of thermal $e t$, we now define the effective coupling $\mathrm{T}_{\text {if }}$ as [14]

$T_{i f}=\left(E_{1}-E_{2}\right) / 2=\left\langle\psi_{1} H^{e l} \psi_{1}\right\rangle \cdot\left\langle\psi_{2} H^{e l} \psi_{2}>/ 2\right.$

where $E_{1}\left(E_{2}\right)$ is the eigenvalue associated with $\psi_{1}\left(\psi_{2}\right)$ in the TS. The diabatic energy matching at the TS (eq (13)) then implies (via eq (12), where we now have 5050 mixing or $\cos \theta=\sin \theta=1 / \sqrt{2}$ ),

$T_{i f}=\left\langle\psi_{i} H^{e l} \psi_{f}\right\rangle$

The sign of $T_{i f}$ is a physical observable, but is contingent on the phase conventions entailed in the definitions of $\psi_{i}$ and $\psi_{f}$ (and hence via eq (12), also $\psi_{1}$ and $\psi_{2}$ ), as discussed in Section $5[14,43]$.

We now consider various approximate methods of evaluating $T_{\text {if }}$, using either the adiabatic $(e q(14))$ or diabatic $(e q(15))$ representation.

\subsection{Superexchange via Perturbation Theory}

The diabatic states $\psi_{i}$ and $\psi_{f}$ as defined in the TSA have the charge involved in the et process localized predominantly on the $\mathrm{D}$ or A sites, respectively, but with tails extending into the bridge region. It is the overlap of these tails which is usually crucial in determining the magnitude of $T_{\text {if. }}$ This role of the electronic manifolds of the bridge in mediating the long-range coupling of $\mathrm{D}$ and $\mathrm{A}$ sites is given precise form in superexchange (se) theories, which were introduced in Section 2.3 and which we now examine in detail in the remainder of the chapter. A detailed understanding of se coupling is achieved by introducing an auxiliary (zerothorder) basis of diabatic states (taken generally as orthonormal), which by construction have the transferring charge entirely localized on the various sites of 
the system (D,A, and the "units" of $B$, as discussed in Section 2). We denote these states as

$\psi_{D}^{\rho} \equiv \psi_{i}^{O}$

$\psi_{A}^{0} \equiv \psi_{f}^{0}$

$\psi_{j}^{B} \quad j=1, n$

where $\mathbf{n}$ is the total number of intermediate bridge states (particular choices of bridge units, $m$ in number, with $m \leq n$, are presented below). These states, which we denote collectively as $\left\{\psi_{\mathbf{k}}^{0}\right\}$, may be considered as eigenfunctions of some suitable 0th order Hamiltonian with 0 th order energies $\mathrm{E}_{\mathrm{k}}^{0}$ :

$H^{e l}=\left(H^{0}\right) e l+V^{e l}$

$\left(H^{0}\right) e l \underset{\psi_{k}}{O}=E_{k}^{0} \underset{\psi_{k}}{0}$

and with vanishing first-order energies (i.e., $\mathrm{v}_{\mathrm{kk}}^{\mathrm{el}} \equiv\left\langle\psi_{\mathrm{k}}^{0} \mathrm{Vel}^{\mathrm{V}} \psi_{\mathrm{k}}^{0}\right\rangle=0$ ).

Eq (15), which involves $\mathrm{H}^{e l}$ and the "full" diabatic states $\psi_{\mathrm{i}}$ and $\psi_{\mathrm{f}}$ (i.e., "dressed" with their long range tails) may now be recast in the equivalent form,

$T_{i f}=\left\langle\psi_{D}^{O}\right| T$ eff $\left|\psi_{A}^{O}\right\rangle$

which implicitly defines the effective transition operator, $T$ eff.

\subsubsection{Results second-order in coupling to the bridge}

We first adopt a "dynamical" point of view and evaluate $T_{\text {if }}$ as given by eq (18), with $\mathrm{T}$ eff taken as the "transition operator" from scattering theory [12],

$T(E)=V^{e l}+V^{e l} G(E) V^{e l}$ 
where $G(E)$ is the electronic Green function

$G(E)=\left(E-H^{e l}+i \varepsilon\right)^{-1}$

(in the remainder of the chapter we suppress the $e l$ superscript).

The Green function depends on the continuous variable $\mathrm{E}$, the "tunneling energy" [12,54], and a positive infinitesimal constant $\varepsilon$, for which the limit $\varepsilon \longrightarrow$ 0 is ultimately taken. In the general case, which could accommodate vibronic effects and the influence of low-lying bridge states beyond the simple TSA defined above, the full Green function (including self energy contributions) should be employed, and $\varepsilon$ may play a crucial role [56]. In the TSA, however, where the D and $\mathrm{A}$ sites are relatively weakly coupled to the bridge, one need only consider the bridge Green function, $G_{B}(E)$, which operates exclusively on the space defined by the $\Psi_{\mathrm{B}_{\mathrm{j}}}^{0}$ (see (16c)),

$G_{B}(E)=\left(E-H_{B}+i \varepsilon\right)^{-1}$,

where $H_{B}$ may be obtained from $H$ using the projection operator for the $B$ space 0 $\left(\left(\Psi_{\mathrm{B}_{\mathrm{j}}}\right)\right.$ and where $\varepsilon$ may be effectively set to zero. Switching to a matrix representation, we approximate eq $(19 a)$ as

$$
T_{i f}(E)=V_{i f}+\sum_{j, k}^{n} V_{i j}\left(G_{B}(E)\right)_{j k} V_{k f}
$$

where $V_{i f} \equiv\left\langle\Psi_{i}^{0} V \psi_{f}^{0}\right\rangle$, etc., and the double sum is over the bridge states $\left\{\psi_{B_{j}}^{0}\right\}$. The optimal choice of the tunneling energy has received detailed attention in the literature [54]. When the $\left\{\psi_{B_{j}}^{0}\right\}$ are well-separated energetically from $\psi_{D}^{0}$ and $\psi_{A}^{0}$, it is adequate to set $E=E_{D / A}^{0}$, the mean value of $E_{D}^{0}$ and $E_{A}^{0}$ (in the general case, when the $\mathrm{D}$ and $\mathrm{A}$ sites of the DBA system are not symmetry-equivalent, the condition 
given by eq (13) does not necessarily correspond to $E_{D}^{0}=E_{A}^{0}[14,32,57]$; see also the results from partitioning theory [11] given below).

Eq (21) displays $T_{\text {if }}$ as a direct or "through-space" $(t s)$ term and a bridge-mediated superexchange term (often denoted "through-bond" $(t b$ ), although formal covalent bonds linking $\mathrm{D}$ and $\mathrm{A}$ via the bridge are not required $[43,48]$ ) which, consistent with the TSA, is lowest (i.e., first) order in $D / B\left(V_{i j}\right)$ and $D / A\left(V_{k f}\right)$ coupling (i.e., overall second order in coupling to the bridge).

Switching now to a stationary point of view, we note that eq 21 can be reinterpreted as

$T_{i f}=\left\langle\stackrel{O}{\psi_{D}} H^{e f f}(E) \stackrel{O}{\psi_{A}}\right\rangle$

where Heff is the effective energy-dependent Hamiltonian derived from Lowdin partitioning theory [11] and defined in the $\psi_{\mathrm{D}}^{0}, \psi_{\mathrm{A}}^{0}$ space. As above, an approximate result (good to second order in coupling to the bridge) is obtained by replacing $\mathrm{E}$ with the 0th-order value, $\mathrm{E}_{\mathrm{D} / \mathrm{A}}^{0}[53]$.

Yet another second-order expression for $\mathrm{T}_{\text {if }}$ is obtained by adopting the adiabatic representation (eq (14)) and evaluating $E_{1}$ and $E_{2}$ using second-order Rayleigh-Schrodinger perturbation theory (RSPT) with the $\left\{\psi_{k}^{0}\right\}$ basis, taking the $\Psi_{B_{j}}^{0}$ as eigenfunctions of $H_{B}$ (i.e., where $V$ in eq 17 a includes only interactions between the bridge and the D/A sites):

$T_{i f}=\sum_{j}\left(V_{i j} V_{j f}\right) / \Delta_{j}^{0}$

where $\Delta_{j}^{0} \equiv E_{D / A}^{0}-E_{B_{j}}^{0}$ (note that these energy gaps are in general negative).

This result, derived by McConnell [47], is equivalent to those given by eqs (21) and (22), if the same $\psi_{B_{j}}^{0}$ basis is employed (i.e., bridge eigenfunctions). In terms of the 
terminology introduced earlier, eq (23) represents the superexchange result when the entire bridge is one "unit" containing all n 0th order states.

It is to be emphasized that aside from the limitation of a second-order treatment of the coupling of $\mathrm{D}$ and $\mathrm{A}$ to the bridge, all of the formulations of $\mathrm{T}_{\mathrm{if}}$ in this section can be implemented in a non-perturbative manner (i.e., the $\left(G_{B}(E)\right)_{j k}$ elements appearing in eq (21), or their counterparts in eqs (22) and (23), may be treated exactly, either explicitly [58,59], or implicitly [60,61]). Eqs (21-23) provide upper limits for the magnitude of $T_{\text {if }}$ as defined by eq (14) [53]. We now apply perturbation theory to the treatment of the bridge and generate higher-order se expressions.

\subsubsection{Higher-order superexchange models.}

Further elaboration of eqs (19a) and (21) may be achieved by invoking the Dyson equation [56,57],

$G(E)=G^{0}(E)+G^{O}(E) V G(E)$

where

$G^{0}(E)=\left(E-H^{0}+i \varepsilon\right)^{-1}$

$\mathrm{Eq}$ (19a) together with eq (24) yields an iterative version of the Lippmann-Schwinger equation [12]

$T(E)=V+V G^{0}(E) T$

As shown by Ratner [12], successive iteration yields $T(E)=V+V G^{0}(E) V \cdots+V\left(G^{0}(E) V\right)^{p-1}$ 
where the pth term is pth order in the coupling V. Adopting the matrix representation in the basis $\left\{\psi_{\mathrm{k}}^{0}\right\}$, including only the bridge component of $\mathrm{G}^{0}(\mathrm{E})$ (cf. eqs (19b) and (20)) with $E=E_{D / A}^{(0)}$ and $\varepsilon$ set to zero,

$\left(G_{B}^{0}\right)_{j k}=\delta_{j k} / \Delta_{j}^{0} j, k=1, n$

and truncating eq. (27) at the value of $p$ which yields convergence to the desired tolerance, allows $T_{\text {if }}$ to be displayed as the superposition (with constructive or destructive interference) of all superexchange "pathways" up to order $p$, subject to the constraint entailed in the use of $G_{B}^{0}$ - i.e., the restriction of first-order dependence on $V_{i j}$ and $V_{k f}$ for $p>1$, leaving up to ( $p$ - 2) th order dependence on intra-bridge coupling elements, $V_{j k}$. Smooth convergence is expected when the ratios $V_{j k} / \Delta_{k}^{0}$ are sufficiently small in magnitude compared to unity.

For example, truncating at $\mathrm{p}=3$ yields

$T_{i f}=V_{i f}+\sum_{j}^{n} V_{i j} V_{j f} / \Delta_{j}^{O}+\sum_{j, k}^{n} V_{i j} V_{j k} V_{k f} / \Delta_{j}^{O} \Delta_{k}^{O}$

Thus the p-th order superexchange pathways include all sojourns from $D$ to $A$ involving stops at $\mathrm{p}-2$ virtual intermediate bridge states, including multiple visits ("retracings" or "backscattering" $[49,50])$ to a given state.

The previous results may be extended to accommodate the case of non-orthogoal $\psi_{B_{j}}^{0}$, with overlap matrix elements $S_{j k} \equiv\left\langle\psi_{B_{j}} \mid \psi_{B_{k}}\right\rangle$. Careful analysis [61] shows that in the general case, the matrix representation of $G_{B}(E)$, introduced in eq $(20)$, should have the diagonal matrix E1 (where 1 is the unit matrix) replaced by ES (see also [12]).

The higher-order results obtained from the "scattering approach" (e.g., eq. (29) can also be obtained by extending the 2nd order RSPT result based on the adiabatic 
splitting (i.e., eqs. (14) and (23)) to the higher order required when the $\left\{\psi_{\mathrm{B}_{j}}^{0}\right\}$ are no longer eigenfunctions of $H_{B}[14,47]$. For the case of a linear homologous sequence of $n$ bridge "units," each with a single state, $\psi_{B_{j}}^{0}$, and coupled only by nearestneighbor (NN) interactions, where

$V_{j k}=t \delta_{j k \pm 1} j, k=1, n$

$V_{i 1}=V_{n f}=T$

$\Delta_{j}^{0}=\Delta$

we obtain the celebrated exponential $n$-dependence predicted originally by McConnell [47] (introduced earlier in Section 2.3),

$T_{i f}=\left(T^{2} / \Delta\right)\left(t / \Delta^{n-1}\right.$

This result of McConnell is actually somewhat restricted [14] since it assumes that the two matrix elements of $\mathrm{V}$ in eq. (30b) have the same sign, and likewise for the elements in eq. (30a). The more general situation, and the possibility of sign alternation with $\mathrm{n}$, are discussed in later sections. Higher-order expressions for $\mathrm{T}_{\mathrm{if}}$ based on the partitioning method (eq. (22)) have also been reported [11].

\section{$3.2 \quad$ Variational Models.}

$\mathrm{T}_{\text {if }}$ may be evaluated entirely in terms of variationally-determined wavefunctions $[14,43]$, either from the adiabatic splitting (eq. (14)), where $E_{1}$ and $E_{2}$ are evaluated separately, or from eq. (15), where charge-localized wavefunctions $\left(\psi_{i}\right.$ and $\left.\psi_{f}\right)$ are obtained directly. An interesting feature of this latter approach is that in general the $\psi_{i}$ and $\psi_{\mathrm{f}}$ so obtained are not orthogonal and thus require use of the more general expression [14] 
$T_{i f}=H_{i f} \cdot\left(S_{i f}\right)\left(H_{f f}\right)$

$T_{f i}=H_{f i} \cdot\left(S_{f i}\right)\left(H_{i i}\right)$

where $S_{i f}=\left\langle\psi_{i} \mid \psi_{f}\right\rangle$ and where the $T$ matrix is Hermitian $\left(T_{i f}=T_{f i}\right)$ due to the assumed energy degeneracy (eq. (13)). Analogous situations in which $S_{\text {if }} \neq 0$ arise in some cases where $\psi_{\mathrm{i}}$ and $\psi_{\mathrm{f}}$ are obtained directly using perturbation theory [14].

\subsection{Extensions and Embellishments of the McConnell Model.}

Before proceeding to the analysis of cases involving units with multiple states and simultaneous electron and hole transfer, we pursue in more detail the nature of the McConnell-type models, extending eq. (31) to the case of a heterogeneous bridge, but retaining the NN coupling model with one state per unit:

$T_{i f}=\left(T_{i 1} T_{n f} / \Delta_{1}^{0}\right) \prod_{j=1}^{n-1}\left(t_{j j+1} / \Delta_{j+1}^{0}\right)$

where the coupling elements and energy gaps are straightforward generalizations of eq. (30), and with $n \geq 1$.

It is not possible to reach simple conclusions about sign patterns without taking account of the many-electron nature of the states and the details of the orbital interactions [14], a topic which we defer to Section 3.4. However, it is of interest to inquire about the utility of the simple form of eq. (33) in treating actual bridges of chemical interest. It is known, for example, that non-nearest-neighbor interactions are very important when superexchange is analyzed in terms of local (i.e., two-center) bonding or anti-bonding orbitals $[37,49,63,64]$. Furthermore, the presence of side chains [62] or the occurrence of "retracing" or "backscattering" $[62,63]$ would also be expected to complicate the coupling, thus perhaps greatly reducing the utility of the concept of a pathway of the type exemplified by eq. (33), 
with its attractive feature of factorization into contributions from each bridge unit. Nevertheless, computational applications of the pathway concept, which exploit the simple form of eq (33), seem capable of giving useful insight into the nature of long-range D/A coupling in complex molecular assemblies [65], thus indicating the utility of the concept at least is an effective sense.

Some progress in rationalizing this situation has been achieved by formulating "renormalized" $t$ and $\Delta$ parameters, which mimic the effects of some of the complications noted above, while allowing the form of eq. (33) to be maintained $[58,62,63,66-69]$. Nevertheless, the "intersection" of different pathways, which becomes increasingly important as the degree of connectivity increases (e.g., as in polycyclic bridges), leads to interference effects which require a departure from the "single effective pathway" picture (eq. (33)) [58], as exemplified by the superposition displayed in eq. (29). When it becomes essential to include multiple states on each site, the form of eq. (33) may still be retained in the sense that scalar multiplication is replaced by matrix multiplication, where the scalar $T(t)$ factors become linear (rectangular) arrays and the $\Delta$ become diagonal square arrays $[11,43]$.

Finally, we note that an exact implementation of eq. (23) for the homologous linear bridge with NN coupling gives an extension of eq. (31) not limited by the $t / \Delta$ $<<1$ constraint [53]:

$T_{i f}=2(-1)^{n}\left(T^{2} / t\right) f_{n}(\alpha)$

where

$f_{n}(\alpha)=g(\alpha) /\left[(\alpha+g(\alpha))^{n+1}-\left(\alpha-g(\alpha)^{n+1}\right)\right]$

$g(\alpha)=\left(\alpha^{2}-1\right)^{1 / 2}$ 
and $\alpha=|\Delta / 2 t|$

Eq (34) is straightforwardly seen to yield the limiting eq. (31) when $|\alpha| \gg>1$. Remarkably, eq. (35) yields nearly exact exponential n-dependence essentially down to $|\alpha|=1$, while oscillations of $T_{\text {if }}$ are observed for $|\alpha|<1$ [53]. A more detailed analysis which included self-energy terms in the bridge Green function (eq. 20), observed a narrow region with $1 / \mathrm{n}$ falloff near $|\alpha|=1$ and eliminated singularities in the $|\alpha|<1$ region [20].

\subsection{Many-particle Perspectives.}

The many-electron wavefunctions pertaining to a DBA system are conveniently represented in terms of 1-electron orbitals. We have already noted (Section 2.2) that the overall many-electron coupling element $T_{\text {if }}$ may often be represented to good approximation as an effective 1-particle quantity (i.e., $\mathrm{T}_{\mathrm{DA}}$ in eq. 8), in which the particle is exchanged between ortibals localized on $\mathrm{D}$ and $\mathrm{A}$. We now take a more detailed look at the coupling by decomposing it into "hole" and "electron" contributions associated, respectively, with the occupied and unoccupied orbitals of B. To illustrate this situation we adopt an independent-particle model for the many-electron Hamiltonian,

$H=\sum_{i} h\left(r_{i}\right)$

and employ single-determinant orbital wavefunctions to represent $\psi_{\mathrm{D}}^{0}, \psi_{\mathrm{A}}^{0}$, and $\left\{\psi_{\mathrm{B}_{\mathrm{j}}}^{0}\right\}$. Relative to the reference occupied bridge manifold in $\Psi_{\mathrm{D}}^{0}$ and $\Psi_{\mathrm{A}}^{0}$, the $\left\{\psi_{\mathrm{B}_{\mathrm{j}}}^{0}\right\}$ involve (in a virtual sense) either removal of electrons from the occupied manifold or addition of electrons to the unoccupied manifold. Not surprisingly the picture of hole and electron contributions depends on the choice of bridge units. 


\subsubsection{Case of the Entire Bridge as One Unit.}

In this case, which corresponds to the 2 nd order expression given by eq. (23), each bridge eigenfunction $\psi_{\mathrm{B}_{\mathrm{j}}}^{0}$ has one hole or one excess electron. Thus, if we define the occupied and unoccupied orbital manifolds as

$\phi_{j}^{h} j=1, n_{o c c}$

$\phi_{j}^{e} j=1, n_{u n o c c}$

the many-electron matrix elements $V_{i j}$ may be expressed in terms of the orbital matrix elements,

$T_{x j}^{h}=\int \phi_{x}^{o} h \phi_{j}^{h}$

$T_{x j}^{e}=\int \phi_{x}^{0} h \phi_{j}^{e}$

where $\mathrm{x} \equiv \mathrm{D}$ or $\mathrm{A}$

and $\phi_{\mathrm{D}}^{0}$ and $\phi_{\mathrm{A}}^{0}$ are the localized $\mathrm{D}$ and $\mathrm{A}$ orbitals. Taking due account of the antisymmetry of a single determinant (i.e., the permutational symmetry) with respect to interchange of electrons $[14,71]$, we may now reexpress eq. (23) in the following orbital form [14],

$T_{D A}=T_{D A}^{h}+T_{D A}^{R}$

where

$T_{D A}^{y}=\sum_{j}^{n_{y}} T_{D j}^{y} T_{j A}^{y} / \Delta_{j}^{y}$

where $\mathrm{y}=\mathrm{h}$ or $\mathrm{e}, \mathrm{n}_{\mathrm{y}}=\mathrm{n}_{\mathrm{occ}}$ or $\mathrm{n}_{\text {unocc, }}$, and $\Delta_{\mathrm{j}}^{\mathrm{y}}$ is the orbital energy gap defined analogously to the many-electron state energy gap introduced in eq (23); note that $\Delta_{\mathbf{j}}^{\mathrm{h}}$ is positive: 
$\Delta_{j}^{h}=\stackrel{O}{\varepsilon_{D} / A}-\varepsilon_{j}^{h}$

$\Delta_{j}^{e}=\varepsilon_{D / A}^{0}-\varepsilon_{j}^{e}$

$\stackrel{O}{\varepsilon_{D / A}}=\stackrel{O}{\varepsilon_{D}}=\stackrel{O}{\varepsilon_{A}}$

Thus a decomposition based on the eigenfunctions of the bridge yields a simple additive superposition of hole and electron pathways, although the question as to whether the interference is constructive or destructive is not immediately obvious [14]. An indirect numerical method for achieving the h/e partitioning (eq. (30)) when the explicit orbital contributions are not available has been reported recently [44].

\subsubsection{Alternative Local Orbital Model.}

To get more insight into the nature of the $\mathrm{h} / \mathrm{e}$ interference, we now adopt an alternative partitioning according to which each of $m$ subunits of $B$ has one occupied $\left(\phi_{j}^{h}\right)$ and one empty ( $\left.\phi_{j}^{e}\right)$ orbital (i.e., $n=2 m$; see comment after eq (16)). A perturbative treatment of the type used in Section 3.1.2 yields the following pure hole and electron contributions in the local orbital framework [14]:

$T_{D A}^{y}=\left(T_{D 1}^{y} T_{n A}^{y} / \Delta_{1}^{y}\right) \prod_{j=1}^{m-1}\left(t_{j j+1}^{y} / \Delta_{j+1}^{y}\right) \quad y=h, e$

where $t_{i j+1}$ is a NN orbital coupling element within the bridge. For a typical case of interest, namely where the $\phi_{j}^{h}$ and $\phi_{j}^{e}$ are, respectively, local bonding and antibonding sigma orbitals, the phases of the orbitals can be arranged so that all the $T^{h}$ and $t^{h}$ elements on the rhs of eq. (42) for the $y=h$ case are normally expected to be negative, whereas the two $\mathrm{T}^{\mathrm{e}}$ factors in eq (42) with $\mathrm{y}=\mathrm{e}$ have opposite signs, while the te's are positive[14]. The important consequence is that: (a) both $\mathrm{T}_{\mathrm{DA}}^{\mathrm{h}}$ and $\mathrm{T}_{\mathrm{DA}}^{\mathrm{e}}$ alternate in sign with $\mathrm{m}$ (i.e., the parity rule, with negative sign (the 
"normal" case) for m even); and (b) $\mathrm{T}_{\mathrm{DA}}^{\mathrm{h}}$ and $\mathrm{T}_{\mathrm{DA}}^{\mathrm{e}}$ interfere constructively. However, this partitioning based on local orbitals is not the same as that based on bridge eigenfunctions (eq (39)). While it may be assumed that $h$ (see eq (36)) does not couple the orbitals on a given unit, coupling is expected between a NN pair, $\phi_{j}^{h}$ and $\phi_{\mathrm{j} \pm 1}^{\mathrm{e}}$ : i.e., the orbital eigenfunctions employed in Section 3.4.1 would involve some mixing of such pairs, and these contributions must be added to the pure hole and electron terms displayed in eq. (42), as illustrated in the next section.

\subsubsection{Inclusion of Hybrid Pathways.}

Continuing with the local orbital model of Section 3.4.2, we consider all possible pathways of the "forward" type (i.e., where an electron moves toward A or a hole moves toward $D$, with no "retracings"). To enhance the multiparticle perspective we proceed in the context of explicit single-determinant wavefunctions.

A rich diversity of NN-pathways is obtained if the occupations of both orbital sets $\left(\left\{\phi_{j}^{e}\right)\right.$ and $\left.\left(\phi_{j}{ }_{j}\right\}\right)$ are allowed to vary, subject to the above assumptions and restrictions. The different types of pathways are illustrated schematically for the case $m=2$ in fig. 4 [43]. It is of particular interest to identify the minimal number of particles (electrons $(e)$ or holes $(h)$ ) necessary to characterize each pathway. Towards this end we define a reference (or "vacuum") configuration and indicate explicitly only those occupation changes relative to it. The resulting virtual transitions are then of four types: $e$ or $h$ transfer (i.e., the passage of an $e$ or $h$ either from or to one of the bridge orbitals $\phi_{j}^{e}$ or $\phi_{j}^{h}$, respectively), and the creation (+eh) or destruction (-eh) of eh pairs. Fig. 4a displays pure e transfer and corresponds to a 1-particle process, whereas the hybrid pathways (figs. 1c and 1d) involve up to 3 particles (both $e$ and $h$ ) relative to the vacuum level. While the process in fig. $4 \mathrm{~b}$ is pure hole transfer (i.e., involving only holes on the bridge 
sites), it is seen to be of the 3-particle type. Since one expects a close correspondence between $e$ and $h$ pathways, the asymmetry of fig. 4a (1-particle) vs. fig. 4b (3-particle) may be surprising, and in fact, the expected isomorphism may be recovered by simply adopting another vacuum level for hole transfer (fig. 5). Thus fig. 4a and fig. 5 bear the expected mirror image 1-particle relationship.

While figs. 4a-d display the four basic types of pathways possible for $m=2$, there are actually a total of six pathways. The other two are variants of fig. $4 \mathrm{c}$ and $4 \mathrm{~d}$ obtained, respectively, by interchanging the first and second virtual transfers in fig. 4c (fig. 4c') and the second and third transfers in Fig. 4d (fig. 4d'). Figs. 1 and 5 include the energy gaps associated with the intermediate states $\psi_{j}^{0}$ (the state and orbital gaps are the same, aside from the sign difference in the case of holes [see eq (41)], in view of the independent - particle model adopted here).

The various contributions to $\mathrm{T}_{\mathrm{DA}}$ are assembled in Scheme I, where once again proper account is taken of the permutation symmetry of the single-determinant wavefunctions ([43]).

\section{Scheme I.}

$\mathrm{T}_{\mathrm{DA}}$ Contribution
a) "e"
$\left(T_{D 1}^{e}\right)\left(t_{12}^{e e}\right)\left(T_{2 A}^{e}\right) /\left(\Delta_{1}^{e} \Delta_{2}^{e}\right)$
b) " $h "$
$\left(T_{D 1}^{h}\right)\left(t_{12}^{h h}\right)\left(T_{2 A}^{h}\right) /\left(\Delta_{1}^{h} \Delta_{2}^{h}\right)$
$c+c$ ') "e $h_{2} h^{\prime \prime}$
$\left(T_{D 1}^{e}\left(t_{12}^{e h}\right)\left(T_{2 A}^{h}\right)\left(-1 / \Delta_{1}^{e}-1 / \Delta_{2}^{h}\right) /\left(\Delta_{1}^{e}-\Delta_{2}^{h}\right)\right.$
$\left.d+d^{\prime}\right)$
"h ${ }_{1} e_{2} "-\left(T_{D 1}^{h}\right)\left(t_{12}^{h e}\right)\left(T_{2 A}^{e}\right)\left(1 / \Delta_{1}^{h}-1 / \Delta_{2}^{e}\right) /\left(\Delta_{1}^{h}-\Delta_{2}^{e}\right)$ 
Summation of the contributions from all six pathways yields the following compact expression,

$T_{D A}=\sum_{\substack{x, y=\\ e, h}}\left(T_{D 1}^{x}\right)\left(t_{12}^{x y}\right)\left(T_{2 A}^{y}\right) /\left(\Delta_{1}^{x}\right)\left(\Delta_{2}^{y}\right)$

Thus the initial evaluation of $\mathrm{T}_{\mathrm{DA}}$ in terms of explicit energy-gaps for the six intermediate many-particle states $\psi_{j}^{0}$ (as displayed in fig. 4 and Scheme 1 ) has been converted to an equivalent expression based on four intermediate 1-particle states in the orbital space, a result which is similar in form to the conventional 3rd-order (for $\mathrm{m}=2$ ) perturbative result (cf. eq (29)). The two diagonal terms in eq. (43), $h h$ and $e e$, correspond to the pure hole and electron processes shown in figs. $4 \mathrm{~b}$ (or 5) and $4 \mathrm{a}$, respectively. The two cross-terms, $e h$ and $h e$, have a less obvious origin, but are clearly seen to be vestiges of the four hybrid processes (figs. $4 c, c^{\prime}, d$, and d'), an insight which underscores the value of the many-particle perspective.

Generalizing to arbitrary $\mathrm{m}$, we find that the 2 -orbital/site NN model yields $2^{\mathrm{m}}$ different pathways, whose relative contributions to $\mathrm{T}_{\mathrm{DA}}$ (both signs and magnitudes) are controlled by the joint action of the orbital parameters $\mathrm{T}, \mathrm{t}$ and $\Delta$. Clearly, the relative importance of the pure electron and hole pathways is expected to diminish with $m$ [43]. Specific computational results for the case of alkyl spacer units will be presented in Section 5, where the one-particle operator $h$ (eq (36)) is identified with the Fock 1-electron Hamiltonian.

In the special case where each $\phi_{j}^{h}, \phi_{j}^{e}$ pair may be taken as linear combinations of a common pair of hybrid atomic orbitals, more insight may be obtained by recasting eq. (43), generalized for arbitrary $m$, as

$\left.T_{D A}=\bar{T}_{D 1} \bar{T}_{m A}\right)\left(1 / \Delta_{1}^{h}-1 / \Delta_{1}^{e}\right) \prod_{j=1}^{m-1} \eta_{j j+1}$ 
where

$\eta_{i j+1}=\left(\bar{t}_{j j+1}\right)\left(1 / \Delta_{j+1}^{h} \cdot 1 / \Delta_{j+1}^{e}\right)$

and where the effective coupling elements $\bar{T}$ and $\bar{t}$ depend on the details of $\phi_{j}^{h}$ and $\phi_{j}^{e}$. For example, if each $\phi_{j}^{h}, \phi_{j}^{e}$ pair is, respectively, a symmetric and antisymmetric combination of symmetry-equivalent hybrid atomic orbitals, then we may write

$\bar{T}_{x y}=T_{x y}^{h}=T_{x y^{\prime}}^{e} x y \equiv D 1$ or $m A$

and

$\bar{t}_{j j+1}=t_{j j+1}^{h}=-t_{j j+1}^{e}$

The factors $\eta_{\mathrm{j}+1}$ associated with each unit of the bridge convey the constructive nature of the $h$ and $e$ interference since $\Delta_{j}^{h}$ and $\Delta_{j}^{e}$ have opposite signs (see eq (41)) $[13,14]$. By analogy with eq (11), $-2 \ln \left|\eta_{j j+1}\right|$ may be considered a local decay coefficient, a quantity dealt with in Section 5.2.

The examples given here in Section 3.4, in spite of the use of a grossly oversimplified NN model, nevertheless serve to show how the role of hole and electron processes in "electron transfer" is somewhat contingent on the choice of the states (or orbitals) used in analyzing the problem (e.g., bridge orbital eigenfunctions vs local orbitals).

\section{More General Models for $\mathbf{T}_{\text {if. }}$}

The discussion of coupling so far has been confined to the TSA for situations of resonant et in the TS, using models limited for the most part to perturbation theory. In this section we introduce more general approaches for formulating 
diabatic states and electronic coupling $\left(\mathrm{T}_{\mathrm{if}}\right)$, which allow these constraints to be relaxed, while remaining within an electronic framework. Consideration of specific vibronic effects may, of course, be required in some cases $[33,72]$.

\subsection{The Mulliken-Hush Model}

Remaining for the moment at the level of the TSA, we invoke the Mulliken-Hush (MH) model for non-resonant coupling [73,74]. Although introduced initially in the weak-perturbation limit $\left(\left|\mathrm{T}_{\mathrm{if}}\right|<<\Delta_{\mathrm{if}}\right.$, the diabatic vertical gap for optical $e t$, displayed as $\mathrm{hv}$ in fig $3 a$ ), it is fundamentally a non-perturbative model, exact within the TSA subject only to the assumption that the off-diagonal dipole moment matrix element between $\psi_{\mathrm{i}}$ and $\psi_{\mathrm{f}}\left(\vec{\mu}_{\mathrm{if}}\right)$ is negligible $[75,76]$. Thus if the adiabatic (a) and diabatic (d) Hamiltonian and dipole matrices are defined, respectively, as

$$
\begin{aligned}
\boldsymbol{H}_{a} & \equiv\left(\begin{array}{ll}
0 & 0 \\
0 & \Delta E_{12}
\end{array}\right) \\
\vec{\mu}_{a} & \equiv\left(\begin{array}{ll}
0 & \vec{\mu}_{t r} \\
\vec{\mu}_{t r} & \Delta \vec{\mu}_{12}
\end{array}\right) \\
\boldsymbol{H}_{d} & \equiv\left(\begin{array}{ll}
0 & T_{i f} \\
T_{i f} & -\Delta i f
\end{array}\right) \\
\vec{\mu}_{d} & \equiv\left(\begin{array}{ll}
0 & 0 \\
0 & \Delta \vec{\mu}_{i f}
\end{array}\right)
\end{aligned}
$$

where the assumption that $\vec{\mu}_{\text {if }}=0$ is imposed in eq. (47b), and where $\Delta \mathrm{E}_{12}$ and $\vec{\mu}_{\mathrm{tr}}$ are, respectively, the energy eigenvalue difference and the adiabatic transition dipole moment difference. Exact solution of the 2-state secular equation yields the following relationship when $\vec{\mu}_{\mathrm{tr}}$ and $\Delta \vec{\mu}_{12}$ are parallel [76]:

$T_{i f}=\left(\left|\vec{\mu}_{t r}\right| /|\Delta \vec{\mu} i f|\right) \Delta E_{12}$

It is customary to evaluate $\left|\Delta \overrightarrow{\mu_{\text {if }}}\right|$ from the relationship

$$
\left|\Delta \vec{\mu}_{i f}\right|=(r D A) e
$$


where $\mathrm{rDA}_{\mathrm{A}}$ is the assumed separation distance between the centroids of the $\mathrm{D}$ and A orbitals (based, for example, on crystal structure data), and where $e$ is the magnitude of the electronic charge.

Thus in optical et, eq (48) provides an estimate of $T_{\text {if }}$ in terms of the vertical excitation energy $\left(\Delta \mathrm{E}_{12}\right)$, the transition moment $\vec{\mu}_{\mathrm{tr}}$ and the assumed $\left|\mathrm{rDA}_{\mathrm{DA}}\right|$. A frequently used expression equivalent to eq. (48) is given by [76]:

$T_{i f}=2.06 \times 10^{-2}\left(\left(\bar{v}_{\max } \varepsilon_{\max } \Delta \vec{v}_{1 / 2}\right)^{1 / 2} / r_{D A}\right.$

where $\left|\vec{\mu}_{\mathrm{tr}}\right|$ has been expressed in terms of the optical parameters $\bar{v}_{\max } \equiv E_{12}(\mathrm{~cm}$. 1), bandwidth $\Delta \bar{v}_{1 / 2}\left(\mathrm{~cm}^{-1}\right)$, and molar absorptivity $\varepsilon_{\max }\left(\mathrm{cm}^{-1} \mathrm{moles}^{-1}\right)$, and where rDA is in $\AA$, and $T_{\text {if in }} \mathrm{cm}^{-1}$.

In fact, the exact 2 -state solution also yields an expression for $\Delta \vec{\mu}_{\mathrm{if}}$ entirely in terms of the elements of $\vec{\mu}_{\mathbf{a}}$ (eq (46b)) [77]:

$\left|\Delta \vec{\mu}_{i f}\right|=\left(\left(\Delta \vec{\mu}_{12}\right)^{2}+4\left(\vec{\mu}_{t r}\right)^{2}\right)^{1 / 2}$

Thus eq. (48), with $\left|\Delta \vec{\mu}_{\text {if }}\right|$ replaced by the rhs of eq. (50), allows $T_{\text {if }}$ to be specified exclusively in terms of adiabatic observables [77]: i.e., $\Delta \mathrm{E}_{12}$ and $\mu_{\mathrm{tr}}$ (obtainable from spectral energy and intensity) and $\Delta \vec{\mu}_{12}$ (obtainable from Stark spectroscopy). Of course, these quantities may also be obtained from quantum calculations. In the limit of resonant et (i.e., $\Delta_{\mathrm{if}}=0$ ) we obtain the expected result (eq. (14))

$T_{i f}=\Delta E_{12} / 2$

since in this case (where $\cos \theta=\sin \theta=1 / \sqrt{2}$ in eq. (12)), $\vec{\mu}$ tr $=\Delta \vec{\mu}_{\text {if }} / 2$. 
In the case of bridge-mediated coupling, the MH model may be generalized using a perturbative superexchange model. As an example, for a single bridge state $\psi_{\mathrm{B}_{1}}^{0}$ we obtain [14]:

$T_{i f}=V_{D 1} V_{1 A} /\left(\Delta_{1}^{O}\right)_{e f f}$

where the effective energy gap is given by

$\left(\Delta_{1}^{0}\right)_{\text {eff }}=2\left(\Delta_{i 1}^{0} \Delta_{f 1}^{0}\right) /\left(\Delta_{i 1}^{0}+\Delta_{f 1}^{0}\right)$

based on the assumption $\left.\vec{\mu}_{11}=\vec{\mu}_{i i}+\vec{\mu}_{f f}\right) / 2$ and with $\Delta_{x 1}^{0} \equiv E_{x}^{0}-E_{1}^{0}, x=i, f$. The latter quantities are vertical gaps based, respectively, on the initial and final states and evaluated at the equilibrium value of the reaction coordinate for $\psi_{i}^{0}$, in contrast to the gaps in Section 3, which pertain to the TS. The variation of gap with reaction coordinate yields a corresponding variation in superexchange coupling $T_{i f}$, which we distinguish with superscripts $e q$ and TS, thus allowing a test of the Condon approximation. We consider for simplicity a thermoneutral process $\left(\Delta \mathrm{G}_{0}=0\right)$, with $\psi_{i}^{0}, \psi_{\mathrm{f}}^{0}$, and $\psi_{\mathrm{B}_{1}}^{0}$ all having parabolic free energy profiles of equal curvature and with the minimum of $\psi_{\mathrm{B}_{1}}^{0}$ and the TS at the same point along the reaction coordinate. We find [78]:

$T_{i f}^{e q} / T_{i f}^{T S}=1-\left(\left(E_{r} / 2\right) /\left(\left|\Delta_{i 1}^{0}\right|\right) \cdot E_{r} / 2\right)^{2}$

where $E_{\mathrm{r}}$ is the reorganization energy (displayed in fig 2). The variation of $T_{i f}$ with position along the reaction coordinate is actually rather modest (e.g., the ratio is > 0.9 for $\mid \Delta_{\mathrm{i} 1}^{0}\left(>2 \mathrm{E}_{\mathrm{r}}\right)$, thus supporting the use of the Condon approximation.

Much of the above analysis rested on the assumption that all dipole vectors are parallel. In general one must adopt a reference direction (e.g., as given by $\Delta \vec{\mu}_{12}$ in 
eq (46b)) and then use the projections of the other dipole matrix elements along this direction [77].

\subsection{Generalized Mulliken-Hush Model.}

We now consider more general models, designed to be broadly applicable to:

(1) ground and excited state processes (either thermal or optical)

(2) multi-state systems (e.g., involving both CS and CR processes; see fig. 3)

(3) general coupling situations (i.e, beyond the weak-perturbation limit)

(4) computational implementation which

(a) allows flexible inclusion of electron correlation

(b) applies to arbitrary geometries (avoiding searches for crossings or seams, thus providing for general tests of the Condon approximation).

We obtain a model satisfying these criteria, denoted as the Generalized Mulliken Hush (GMH) model [78], by exploiting the full consequences of the MH model, recognizing that one of its crucial assumptions, namely that transition moments connecting diabatic states localized at different sites are zero $\left(\bar{\mu}_{\text {if }}=0\right)$ provides a general method for defining diabatic states in terms of purely adiabatic quantities. Specifically, we take the transformation that diagonalizes the adiabatic dipole moment matrix as the transformation to the Mulliken-Hush diabatic states. When one applies this to the adiabatic (diagonal) Hamiltonian, the diabatic Hamiltonian is obtained (previous examples of the use of diagonalization of the dipole moment matrix to define diabatic states in a pair-wise fashion can be found in [79-82]. 
When the multi-state GMH transformation as defined here yields more than one diabatic state localized on the same site, we impose the additional condition that the blocks of the Hamiltonian associated with a single site be diagonal, thus yielding states diabatic in the GMH sense with respect to inter-site coupling, but "locally adiabatic" within each site or local region [77]. The GMH analysis employs the component of each dipole vector in the direction defined by the dipole difference for the initial and final adiabatic states (two-state case) or by the average of such differences when several et processes are considered for a given system.

As already noted above in connection with the 2-state $\mathrm{MH}$ model (now recognized as a special case of GMH), we emphasize that the GMH method can be implemented solely in terms of experimental quantities. Observed excitation energies, transition dipoles from intensity measurements, and adiabatic dipole moment differences from Stark measurements yield direct experimental estimates of diabatic coupling elements according to the above procedure. At the multistate level, the relative sign relations among the transition moments must be known, and may be taken from calculations if not available from analyses of the experimental data. (These phase relations are "observables," as distinct from phase "conventions;" e.g., for a three state system two distinct cases arise: an even or odd number of positive transition dipole moments.) Finally, we mention that as in the 2-state case (Section 4.1), the diagonal GMH diabatic dipole moment matrix directly yields estimates of the centroids of the different states of the system of interest and the associated rDA values, quantities of great utility is interpreting charge-transfer in terms of the results of Stark spectroscopy or quantum calculations $[77,83]$.

One of the central physical assumptions underlying the GMH model - the diagonal form of the diabatic dipole moment matrix - has been given quantitative 
support by considering alternative formulations of diabatic states which take detailed account of the eigenvectors obtained from multistate quantum chemical calculations [84]. These techniques are in the spirit of the so-called "least-motion block diagonal" formulations $[85,86]$.

\section{Computational Applications}

The theoretical models discussed in Sections 3 and 4 have played an important role in quantum chemical applications to a wide variety of chemical systems. The power of current-day computational capability makes molecular systems of considerable complexity (including assemblies with up to $\sim 100$ atoms and several hundred electrons), accessible to detailed electronic structural treatment, using either $a b$ initio or semiempirical techniques [14]. These techniques range from simple pathway approaches $[57,66]$ to 1-electron path integral methods [87] (which permit molecular level treatment of the medium, but which include only "electron" (and not "hole") contributions to $\mathrm{T}_{\mathrm{if}}$ ), to many-electron models of the independentparticle [50,61], self-consistent field (SCF) $[34,36,43,45,64]$, or configuration interaction (CI) type. The CI methods, as well as related perturbative many-body techniques, allow the inclusion of electron correlation $[64,77,88,89]$ and are generally essential for an even-handed treatment of a manifold of different electronic states [77]. Density functional theory (DFT) techniques may also be applied to et systems [89].

Up to the present, most electronic structure calculations applied to et processes have been restricted to isolated DBA systems. Aside from $T_{i f}$, these calculations permit the evaluation of the contribution to reorganization energy from discrete vibrational modes of DBA [90]. Some recent treatments have included the effect of a solvent reaction field, using cavities of realistic shape which take detailed 
account of the molecular structure of the "solute" [52] (i.e., the DBA system). In the cases studied with these realistic models, solvent does not have a significant influence on $\mathrm{T}_{\text {if }}$ magnitudes, although the available data base is small.

A technical problem arises in cases of DBA systems with negative charge (either with or without solvent present), since calculations often do not yield stability with respect to loss of an electron. Yet even for such "unbound" systems, SCF techniques typically yield wavefunctions from which reasonable $T_{\text {if }}$ values may be obtained, provided that the orbital basis does not contain very diffuse functions [34].

In the remainder of this section we focus on various DBA systems with electronically saturated bridges, and present a selective set of results and conclusions from detailed quantum calculations chosen to elicit the key factors which control D/A coupling, both in terms of trends in $\mathrm{T}_{\text {if }}$ values and the analysis of these trends made possible by the theoretical concepts associated with the superexchange model developed in Section 3. Thus we examine the dependence of $\mathrm{T}_{\text {if }}$ on a number of chemical factors which characterize the DBA systems, including
a) charge type
b) symmetry of $\mathrm{DB}$ and $\mathrm{BA}$ interaction
c) conformation of $\mathrm{D}$ and $\mathrm{A}$ relative to $\mathrm{B}$
d) internal conformation of $B$
e) covalent vs H-bonded or non-bonded interactions within DBA
f) energy gaps $(\Delta)$ 
g) role of surrounding solvent

The role of the superexchange model is two-fold: first to provide illuminating decompositions of $T_{\text {if }}$ into compact sets of elementary constituents; and second, to offer a basis for fashioning these constituents into transferable parameters which may be used, with suitable tuning, to design new systems of desired specifications from reference systems of known properties.

A word is in order concerning the correspondence between estimates of $T_{\text {if }}$ obtained from theoretical calculations and from experiment. The course of action advocated in the previous paragraph is very timely precisely because the diverse body of results available at present indicates that the degree of correspondence is indeed quite good, certainly at the semi-quantitative level (some specific comments are offered below). Precise quantitative comparisons remain difficult, not only because of remaining deficiencies in the electronic structure models (and associated treatment of vibronic and medium effects), but also because of the difficulties inherent in extracting $\mathrm{T}_{\text {if }}$ values from experimental kinetic data.

\subsection{Orbital Models}

The results reported below are based on SCF orbital wavefunctions, carried out either at the single (Sections 5.2 and 5.3) or multi (Section 5.4) configuration level. The results are all of the $a b$ initio type, except for Section 5.4, which includes some conventional (non-SCF) CI used in conjunction with the semiempirical INDO/S method [91].

The single-configuration SCF model has been applied to $T_{\text {if }}$ evaluation at three distinct levels, distinguished by the degree of state-specific (i.e., with respect to $\psi_{i}$ and $\psi_{f}$ ) electronic relaxation included [14,43]: 
a) fully-relaxed SCF (charge-localized $\psi_{i}, \psi_{f}$ )

b) symmetrically-delocalized SCF ( $\psi_{1}$ and $\psi_{2}$ (eq. 12 ), with mean-field relaxation)

c) frozen orbitals for $n$-electron DBA system (based on ( $\mathbf{n} \pm 1$ )-electron SCF parent)

These three levels lead, respectively, to evaluation of $T_{\text {if }}$ as

a) direct coupling (eq. (32))

b) adiabatic splitting (eq. (14))

c) adiabatic splitting (eq. (14)) via Koopmans' theorem [92] (i.e., orbital energy splitting)

The performance of the three levels has been thoroughly discussed in the literature $[14,34,43,45,49,64,88]$. The methods are most easily applied to symmetrical DBA systems, since the symmetry helps to specify the reaction coordinate at the TS, where most applications have been made (in this situation, the fully-relaxed SCF wavefunction is spatially symmetry-broken). Treatment of non symmertical systems is also feasible [36,93,94]. The frozen-orbital/Koopmans' theorem (KT) approach is attractive for purposes of analysis, since both initial $\left(\psi_{i}\right)$ and final $\left(\psi_{f}\right)$ states may be characterized by a common set of orbitals, conveniently chosen to correspond to the desired assignment of bridge subunits, as discussed in Section 5.3. Fortunately, the KT model yields results which are generally in reasonable agreement with the two SCF models, at least for purposes of semiquantitative evaluation and analysis. The 1-particle model based on KT may be considered as a particular example of the generic orbital model for $\mathrm{T}_{\mathrm{ij}}$ derived in Section 3.4 (eq (43)) in the context of a set of many-electron configurations. 
With respect to the delocalized, spatially-restricted SCF level (for symmetrical DBA systems), the charge-localized model may be viewed as introducing electron correlation (associated with the charge-state-specific relaxation). More elaborate incorporation of electron correlation has also been investigated (using both manybody-perturbation theory and MCSCF models), and found to make appreciable contributions in some cases, although generally not having a major effect on $\mathrm{T}_{\text {if }}$ magnitudes $[64,88,89]$.

The approaches outlined above have been implemented at the $a b$ initio level with a variety of orbital basis sets, including mimimal (e.g., STO-3G), split-valence (e.g., 3-21G), and extended (e.g., 6-31G*) types [34,43,49,63,64,95]. The 3-21G basis is generally found adequate, and even the minimal STO-3G set is able to give a semi-quatitative account of many trends.

\subsection{Trends in $T_{\text {if }}$ for Saturated Bridges.}

We now consider the results of calculations for families of DBA systems with saturated organic bridges, which illustrate how $T_{\text {if }}$ varies with $D / A$ separation, molecular conformation, nature of the coupling between the units of the system, and degree of solvation. While many other bridge types are of interest, an examination of electronically saturated bridges is especially revealing, showing how prior naive pictures of such bridges as merely inert insulating "spacers" have given way to a detailed appreciation of their effectiveness in facilitating D/A coupling.

\subsubsection{Decay Coefficients ( $\beta$ ) for Homologous Bridges.}

Coupling through trans-staggered alkane bridges in $\left(\mathrm{CH}_{2}\right)_{\mathrm{m}+3}^{ \pm}$radical cations and anions, $1 \pi(\mathrm{m})$, as in fig 6 , is displayed in fig. 7 at three different levels (see Section 
5.1): KT energy splittings (eq. (14)), energy splittings (eq(14)) for SCF delocalized wavefunctions (SCF/deloc), and direct interaction (eq. (32)) of charge-localized wavefunctions ( $\mathrm{SCF} / \mathrm{hoc})$. The calculations are all based on symmetrical $\left(\mathrm{C}_{2 \mathrm{v}}\right.$ or $\mathrm{C}_{2 h}$ ) molecular structures, which may be taken as representative of the transition state for activated $e t[43]$. In each DBA system, D and $\mathrm{A}$ are the terminal $\mathrm{CH}_{2}$ groups, and $m$ denotes the number of $\mathrm{CC}$ bonds within the $\left(\mathrm{CH}_{2}\right)_{\mathrm{m}+1}$ bridge (the sigma bonds linking $\mathrm{D}$ and $\mathrm{A}$ to $\mathrm{B}$ have a minor influence on the overall $\mathrm{D} / \mathrm{A}$ coupling [43]). Consistent with the discussion in connection with eq (8), the $T_{\text {if }}$ values based on the most fully - relaxed wavefunctions ( $\mathrm{SCF} / \mathrm{loc}$ ) have the smallest magnitudes. It is seen that the overall variation of $T_{\text {if }}$ (in the displayed $m=2-7$ range) is roughly exponential (linear regression coefficients, $r$, for $\ln \mid$ Tif $\mid$ fits to $m$ are $>0.98$ [43]), even though local fluctuations are evident (see also [63] and [95]. In studies of longer alkane chains (with a variety of $\mathrm{D}$ and $\mathrm{A}$ groups) a tendency toward flattening of the falloff with increasing chain length has been observed for radical cations [63]. The two SCF models (loc and deloc) give similar results, with $\beta$ slightly greater for radical anions, while the "one-electron" (KT) model gives an appreciably smaller $\beta$ value for the radical cations. Calculated $\beta$ 's in the range $0.7-0.9 \AA^{-1}$ are compatible with estimates $(\sim 0.7-1.0 \AA)$ inferred from experimental kinetic data (see Table $\mathrm{V}$ of [43]). As discussed below, the quasi exponential falloff by no means implies that the simple McConnell type model (eq. (23)) is valid. (Here and in the remainder of the chapter we suppress the se superscript for $\beta$ introduced in eq (11).)

Turning now to more complex bridges comprising bicyclo [1.1.1] pentane units ("staffanes" $[43,63])$, depicted in fig. $8 \mathrm{a}$, we display $T_{\text {if }}$ and $\beta$ values for 1-3 membered bridges in Table 1 (at the KT level). For the $\pi$-type D/A systems $(2 \pi(m)$, where $\mathrm{m}$ denotes the number of bicyclo units, the decay characteristics are similar 
to those displayed by the $1 \pi(\mathrm{m})$ systems. For the analogous $\sigma$-type D/A systems $(2 \sigma(m))$, the limited data suggests a greater difference in falloff for cation us anion systems (note also the different sign pattern, in comparison with that for $2 \pi(m)$ ). The $2 \sigma(1)$ cases have been omitted, since the coupling for them is dominated by direct through-space ( $t s)$ D/A coupling [43,49]. Throughout this chapter, as in refs [43] and [49], the coupling is presented as $-\mathrm{T}_{\mathrm{if}}$, for which a positive sign corresponds to "normal" coupling (see also footnote b in Table 1).

Finally, we emphasize that the type of falloff exemplified by the data of fig. 7 and Table 1 is not limited to DBA systems fully linked by covalent bonds [34,43,95]. Figure 9 displays 1-3 membered bridges $(4 \sigma(\mathrm{m}))$ comprising methane molecules in

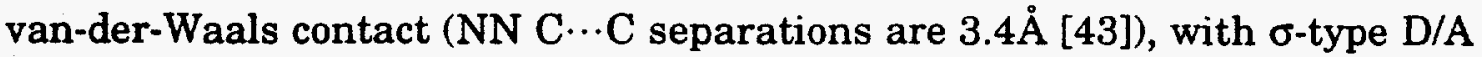
coupling represented by $\mathrm{CH}_{3}$ groups in van der Waals contact with $\mathrm{B}$. Table 2 shows falloff for coupling in the radical anions quite similar to that found for the covalent systems, while the falloff is somewhat faster for the radical cations. Estimates of $\beta \sim 1.2 \AA^{-1}$ for both electron and hole transfer through non covalentlylinked glassy media have been obtained from experimental kinetic data $[48,96]$, with some correlation observed between $\beta$ and the effective energy gap $(\Delta)$ [96].

Table 2 also shows similar decay through non-bonded radical cation systems in which $\mathrm{CH}_{4}$ is replaced by $\mathrm{H}_{2} \mathrm{O}$ (the results for the corresponding radical anions are omitted, since they were found quite sensitive to orbital basis set; this may be a manifestation of the unbound nature of the model radical anion calculations, as noted in Section 5.1).

\subsubsection{Conformational Dependence.}

Since $T_{\text {if }}$ is now thought to be dominated by non-nearest-neighbor interactions, when cast in terms of local bonds (and anti-bonds) $[34,43,49,63,64,97]$, sensitivity to 
molecular conformation is expected [43,49]. Table 3 displays results [49] for conformational variants of $1 \pi(3)$, both with regard to D/A conformation relative to $B$ and the internal conformation of B. Clearly the effects are quite pronounced, involving both sign and magnitude. Indeed these results underscore the importance of considering the sign (a bona-fide observable $[14,43,49]$ ) as well as magnitude, in any attempt to gain a comprehensive understanding of the nature of $\mathrm{D} / \mathrm{A}$ coupling. It is interesting to note that for both $\mathrm{D} / \mathrm{A}-\mathrm{B}$ conformations, the trans $\rightarrow$ gauche $\rightarrow$ cis modification of B always results in a monotonic increase in the algebraic value of $T_{\text {if }}$ (the signs, albeit observables, are nevertheless contingent on the underlying sign conventions, as noted in the table footnotes). It should be emphasized that the "in-plane" and "perpendicular" orientations of D/A orbitals relative to B lead to coupling mechanisms of essentially different qualitative types [49]: in the former case, coupling is dominated by the $\mathrm{CC}$ framework (even though $\mathrm{CH}$ bonds make appreciable contributions), whereas in the latter case, coupling to the $\mathrm{CH}$ bonds becomes either an important (gauche $\mathrm{B}$ ) or the exclusive (trans or cis B) mechanism.

Table 4 displays the effect of staggered $v s$ eclipsed conformation of the two bicyclo units in $2 \pi(2) 2 \sigma(2) 3 \pi(2)$, and $3 \sigma(2)$ The staggered conformers are displayed in figs. 8 and 9. One would expect the bicyclo [2.2.2] systems to be more sensitive than the bicyclo [1.1.1] systems, because in the former case, the relevant pairs of adjacent bonds involve closer contacts due to the larger bond angles at the bridge heads [43]. However, the lack of sensitivity for $3 \sigma(2)$ is surprising.

\subsubsection{Dependence of $T_{\text {if }}$ on the Nature of the Bonding in DBA.}

We have already seen above that similar decay of $T_{\text {if }}$ with $D / A$ separation is observed whether the $\mathrm{D}, \mathrm{A}$ and bridge units are covalently bonded or in non-bonded 
contact, a result which is not surprising in view of the dominat role of non-nearestneighbor interactions noted above. In Table 5 we compare results for covalently linked systems and hydrogen- bonded (H-bond) systems. The H-bond is thought to play an important role in many cases of long-range D/A coupling, especially in protein-mediated coupling [65].

Each of the DBA systems in Table 5 possesses two 3-bond linkages, either fully covalent linked, as for the boat conformer of cyclohexane $(5 \pi)$, or hydrogen bonded, as in the carboxylic acid dimer, $6 \pi$. As expected from Section 5.2 .2 , the results are quite dependent on the conformation of $\mathrm{D}$ and $\mathrm{A}$ relative to $\mathrm{B}$. However, it is clear that the strength of coupling through the H-bonds is comparable to that through a sequence of $\mathrm{CC}$ bonds, even though the $\mathrm{rDA}$ separation is greater in the former case. The carboxyl group, of course, represents an exception to our focus on fully saturated bridges. However, the $\pi$-bond of the carboxyl does not appear crucial here, since the H-bond mediated coupling is actually much more favorable for the "in-plane" orientation of the D/A orbitals, which involves only overlap with the sigma framework of the bridge.

\subsubsection{Influence of Solvent.}

The selection of the "bridge" in any model for $\mathrm{T}_{\text {if }}$ is guided by the assumption that it will give adequate account of all important pathways for superexchange coupling. Relative to a given DBA system in isolation, one might expect (see Section 2) an influence from the surrounding environment (taken here as solvent) either through modulation of the importance of already existing pathways through $B$ by perturbing the relevant energy gaps $\Delta$ (see Section 3 ), or by providing additional pathways by virtue of its own electronic manifolds. As an example, we return to the trans-staggered alkane spacer $(1 \pi(\mathrm{m}))$ and compare in Table 6 results 
for $\beta$ obtained: 1 ) in vacuo; 2 ) with an aqueous medium represented by a realistic molecular cavity in a dielectric continuum [52]; and 3) with explicit addition of water molecules (see structure 7) in the quantum chemical calculations [98]. Solvent is seen to exert no influence on $\beta$ via the long-range interaction provided by the dielectric continuum model [52]. Even with the enlarged superexchange model entailed in the inclusion of water molecules, the only effect is a very modest reduction in $\beta$ for radical cations. Qualitatively, this result is not surprising, since the relatively high-lying lone pair orbitals of $\mathrm{H}_{2} \mathrm{O}$ are expected to participate (at least to some extent) in hole-type superexchange (most important for coupling in radical cations), whereas the unoccupied water orbitals are not expected to be effective in promoting corresponding "electron"-type pathways. Aside from $\beta$ values, the magnitudes of $T_{\text {if }}$ are essentially unaffected by inclusion of a polarized dielectric [52], and even with the explicit waters the effects are minor $(<10 \%)$.

5.3 Analysis of Superexchange Coupling in Terms of Natural Bond Orbitals (NBO's).

Considerable insight into the nature of superexchange coupling through saturated bridges has been obtained in an additive perturbative framework by adopting 2 center "bridge units" containing localized "bond" and "anti-bond" molecular orbitals (LMO's). These represent an effective compromise [43] between even more localized (1-center) atomic orbitals (which interact too strongly to make perturbation theory applicable) and larger molecular fragments (amenable to a perturbative threatment, and of demonstrated utility [11,50], but less obviously likely to provide a basis for transferable parameters). A convenient, (though by no means unique) definition of LMO's is the natural bond orbital (NBO) scheme of Weinhold and coworkers [99]. Detailed studies [49,63,97] have shown that NBO's yield a workable perturbative scheme (e.g., along the lines of eqs. (29)), in which overall $\mathrm{T}_{\text {if }}$ magnitudes at the 1-electron $\mathrm{KT}$ level, may often be recovered additively 
at a level of sufficient quantitative accuracy to make a detailed NBO-based mechanistic analysis of great value. These treatments are all within the "second order" constraint of the TSA (Sections 2 and 3), which precludes intermediate return "visits" to the $\mathrm{D}$ site.

\subsubsection{NBO Gaps and Hopping Integrals.}

We display in Tables 7 and 8 typical values, respectively, of gaps $(\Delta j)$ and hopping integrals $(\mathrm{T}, \mathrm{t})$ in the NBO basis obtained from the Hartree-Fock MO's of the neutral parent (i.e., the frozen orbitals which define the KT model). For radical anions, the $|t / \Delta|$ ratios are all well below unity, whereas for radical cations, $|t / \Delta|$ is seen to approach 0.5 in some cases. Thus for these cations, smooth convergence certainly cannot be taken for granted, even though it is often found in practice when $\mathrm{D}$ and $\mathrm{A}$ groups are modeled by $\mathrm{CH}_{2}[43,49,63,97]$ or other [63] groups. Chemical variation of the D/A groups may, of course, substantially reduce the NBO gaps $(\Delta)$. In such cases [34,63], model 1-electron Hamiltonians in an NBO basis are still of value even if perturbation theory must be abandoned.

The sharp disparity in magnitudes of $\mathrm{t}_{12}$ for CC and CC* NBO's (Table 8) may be qualitatively understood in terms of the different nodal structure of the respective NBO's [49]: primarily constructive (destructive) interference pertains when adjacent $\mathrm{CC}\left(\mathrm{CC}^{*}\right)$ bonds interact, as shown schematically in fig. 10.

\subsubsection{NN vs Non-Nearest-Neighbor Coupling.}

The order-of-magnitude difference in $\mathrm{NN} t$ values does not lead to as much disparity in hole $v s$ electron coupling as one might expect, because of the relatively minor role which NN interactions play in overall coupling [34,49,97]. A qualitative sampling of this situation is offered in fig. 11, which displays the most important 
individual pathway (i.e., the contribution to the perturbation sum of greatest magnitude) for ten different DBA systems, characterized by $\pi$ and $\sigma$-type D/A groups coupled to various acyclic, cyclic and bicyclic bridges (obtained for radical anions at the 3-12G/KT level) [49]. These pathways all involve the carbon framework, but in only one case (the first entry on the left) does a NN McConnell path appear, while the other cases involve jumps over at least one link in a covalent sequence (see also the similiar analyses in [34] and [64]). For the $\pi$-type D/A cases, coupling between D/A groups and the sigma bonds linking them to the bridge is very weak, a consequence of the $\pi-\sigma$ orthogonality $[49,63,64]$. In larger bridges $[63,64]$, pathways involving jumps over 1-3 NN covalent links are typically found to be the most important.

It is to be emphasized that the "most important" pathways are generally not dominant (and in fact may have the sign opposite to that of the overall $\mathrm{T}_{\text {if }}$ [49]), since $T_{\text {if }}$ is generally the superposition of a very large number of competing terms, especially when the extended orbital basis sets desirable for adequate variational flexibility are employed $[49,64]$. In this sense, the NBO units are too "finegrained," and a "coarser-grained" selection of bridge units would be necessary to obtain a very compact representation of $\mathrm{T}_{\text {if }}$ (i.e., a small set of effective pathways).

\subsubsection{Trans-staggered Bridges $(1 \pi(m))$.}

A decomposition of $T_{\text {if }}$ into hole, electron, and hybrid contributions for radical cations and anions of $1 \pi(\mathrm{m})$ is presented in Table 9. For simplicity, we consider only CC and CC* contributions obtained at the STO-3G/KT level [43]. Comparable results have been obtained with inclusion of $\mathrm{CH}$ and $\mathrm{CH}^{*} \mathrm{NBO}$ 's and a larger (i.e., 3-21G) basis set, although quanitative differences have been noted [64]. While pure 
hole pathways are always more important than pure electron pathways for coupling in radical cations, and vice versa for radical anions, we see that the hybrid pathways tend to increase in relative importance as the bridge is lengthened.

An attempt to incorporate the complexity of the pathways for $\pi$-type $\mathrm{D} / \mathrm{A}$ coupling mediated through a trans-staggered alkane bridge in terms of an effective or renormalized McConnell model has been reported for radical cations in [63]. With a model restricted to $\mathrm{CC}$ bonds and pathways of the "forward" type (i.e., no "retracings," even though these in fact make appreciable contributions to $T_{\text {if }}$ ), numerical coefficients were determined such that the scaled McConnell model reproduced the full perturbative result within the model adopted. The scaling coefficients displayed in Table 10 reflect in a compact manner the growth in number of actual pathways which contribute to the effective McConnell pathway. The scale factor is given approximately by

$f_{m} \sim(1.6)^{m-2}$

Further systematic work of this type should be of help in bridging the current gap between effective pathway models [65], and those based explicitly on detailed orbital calculations.

Considering now the effect of varying the D/A group, keeping the bridge constant, we summarize in Table 11 results at the 3-21G/KT level [63], which display the correlation between the $\mathrm{D} / \mathrm{A}$ energy (either the absolute $\mathrm{E}_{\mathrm{D} / \mathrm{A}}^{0}$, or the mean gap relative to $B$ ) and the decay coefficient $(\beta)$ for transfer in radical cations (assumed to be primarily of the hole type). The overall trend is basically monotonic in the sense expected from the simple McConnell-type model (eq. (11)), assuming a common effective $t$ and terminal hopping integral $T$ (see eq. 31 ) for all $D / A$ cases. 


\subsubsection{A Simple Test of Additivity}

In Table 12 we examine the possibility of simple additivity relations for radical anions with $\sigma$ or $\pi$-type coupling through bridges with a variable number of cis 3 bond linkers [49]. Only the through-bond $(t b)$ component is considered (obtained by subtracting the NBO through space $(t s)$ term from the total $\mathrm{KT} / 3-21 \mathrm{G}$ result for $\mathrm{T}_{\text {if }}$. The $t$ s term makes a substantial, and essentially (within 10\%) constant contribution to $\mathrm{T}_{\text {if }}$ for the three $\sigma$ cases $[43,49]$. The contrast between patterns for $\sigma$ and $\pi$ coupling is striking: for $\sigma$ coupling, the $t b$ terms are simply proportional to the number of linkers, whereas, no such pattern is displayed for $\pi$ coupling (even taking due account of direction cosines governing the orientation of the D/A $\pi$ orbitals relative to each linker). This situation is at least superficially compatible with the "most important" pathways shown in fig. 11: i.e., they are all of the same type for $\sigma$ coupling. However, as emphasized above, these pathways are not dominant. In an analogous study of norbornyl-based bridges [100], specific interference between linkers has been elucidated in terms of NBO-based pathways, and in general such effects are expected to disrupt simple additivity schemes for treating multiple.linkers or pathways (see also $[101,102]$ ).

\subsection{Applications of the GMH}

The final set of applications deals with systems involving optical or excited state $e t$. These systems allow many of the general features of the GMH (Section 4) to be exploited

\subsubsection{Et in the 4-state $\mathrm{Zn}_{2}\left(\mathrm{OH}_{2}\right)+$ Manifold}

The $\mathrm{Zn}_{2}\left(\mathrm{OH}_{2}\right)+$ cluster (see figure (12)) provides a model system which allows several features of $\mathrm{D} / \mathrm{A}$ coupling of generic interest to be probed in detail. The four 
lowest-energy electronic states (all spin doublets) are dominated by the four valence-bond structures,

$Z n\left(s^{2}\right) / Z n+(s)$

$Z n^{+}(s) / Z n\left(s^{2}\right)$

$Z n(s \rho \sigma) / Z n+(s)$

$Z n+(s) / Z n(s \rho \sigma)$

where $\mathrm{s}$ and $\rho \sigma$ refer to the valence $4 \mathrm{~s}$ and $4 \rho \sigma \mathrm{Zn}$ orbitals. Complexing an axial water molecule to one $\mathrm{Zn}$ atom, on the side opposite to the distant $\mathrm{Zn}$, provides an additional degree of freedom ( $\mathrm{rZn} 0$ ), which allows the homonuclear symmetry to be broken in a controlled fashion. The relative energies of the four low-lying adiabatic states (obtained by state-averaged $a b$ initio CASSCF calculations [77]) are displayed in fig. 12 for three different values of $\mathrm{rZnO}_{0}$, with $\mathrm{rZnZn}_{\mathrm{n}}$ fixed at $5.0 \AA$. It is not immediately clear how to extract from the 4-state adiabatic picture, the effective coupling elements which govern the four possible et processes among the diabatic states represented schematically in (57): these processes comprise thermal ground state et $(57 \mathrm{a} \rightarrow 57 \mathrm{~b})$; excited state et $(57 \mathrm{c} \rightarrow 57 \mathrm{~d})$, and two optical processes $(57 \mathrm{a} \rightarrow 57 \mathrm{~d}$ and $57 \mathrm{~b} \rightarrow 57 \mathrm{c})$. For completeness we note that the other possible optical processes $(57 \mathrm{a} \rightarrow 57 \mathrm{c}$ and $57 \mathrm{~b} \rightarrow 57 \mathrm{~d})$ involve local excitations of weak intensity because of the singlet $\rightarrow$ triplet character of the local transitions (corresponding to the designation of the excited states as "trip-doublets" [103]). In the following we denote orbitals on the hydrated $\mathrm{Zn}$ with a prime.

Analysis of the 4-state system defined above by use of the GMH model (Section 4.2) in conjunction with the full adiabatic dipole matrix and the state energies yields straightforwardly the results given in Table 13. The strength of 
coupling and the decay coefficients $(\beta)$ are seen to display a sharp dependence on the character of the orbital pairs nominally involved in the four different processes (see above) and on the closeness of the perturbing water molecule (only in the limit of large $\mathrm{rZn}_{\mathrm{n} 0}$ do the $\mathrm{sp}^{\prime}$ and $\mathrm{ps}^{\prime}$ processes become equivalent). The variations with rZno (i.e., departure from the Condon approximation) are complex, displaying examples of non-monotonic dependence. Since the $\mathrm{Zn}_{2}\left(\mathrm{OH}_{2}\right)^{+}$complex lacks a "bridge," the coupliing is direct or $t s$, and indeed, the $\beta$ magnitudes are comparable to those found for coupling through vacuum $([14,28,95]$ and correlate well with the ionization potentials (IP) of the corresponding neutral parents (i.e., as given by eq. (10), with $\Delta^{G}$ replaced by IP). Attempts to approximate the results of the 4-state analysis (Table 13) by a conventional pairwise approach (i.e., successive applications of the 2-state GMH to pairs of adiabatic states), yield increasingly large departures from the 4-state results as $\mathrm{rZnZn}^{\prime}$ decreases: e.g., for $\mathrm{rZnZn}^{\prime}<6 \AA$ departures of up to $50 \%$ in $\mathrm{T}_{\text {if }}$ magnitudes are found. Furthermore, in the limit of weak hydration (i.e., large $\mathrm{r} \mathrm{Zn} 0$ ), treatment at the level of the two-state approximation (TSA) is simply incapable of accounting for the two distinct optical processes ( $\mathrm{sp}^{\prime}$ and $\mathrm{ps}^{\prime}$ ).

\subsubsection{Symmetry and Solvent Effects}

We now apply the GMH approach to the 3-state problem defined by the ground (DBA), locally excited ( $\left.D^{*} B A\right)$, and charge-transfer $\left(D^{+} B A^{*}\right)$ states of the two systems depicted in fig. 13 (8 and 9), where $D$ and $A$ are, respectively, anthracene and olefin moieties, fused to bridges with frameworks comprising norbornyl and cyclobutyl units. Due to the orbital symmetries pertaining to an assumed $\mathrm{C}_{\mathrm{s}}$ plane, the charge separation, $\mathrm{CS}\left(\mathrm{D}^{*} \mathrm{BA} \rightarrow \mathrm{D}^{+} \mathrm{BA}^{-}\right)$, and charge recombination, $\mathrm{CR}\left(\mathrm{D}^{+} \mathrm{DB}\right.$ 
$\rightarrow$ DBA), processes are, respectively, symmetry-forbidden and symmetry-allowed [104]. In this study, the adiabatic dipole matrix and state energies were obtained from INDO/S CI calculations [91], designed to provide a "balanced" treatment of the $\mathrm{D}^{*} \mathrm{BA}$ and $\mathrm{D}^{+} \mathrm{BA}^{-}$excited states by including in the $\mathrm{CI}$ all single excitations from the highest occupied MO (homo) of the ground state. In order to mimic the influence of solvent, the DBA structures were first optimized (at the MM2 force field level [105]), starting with a small sample of assumed interaction geometries between a given solvent molecule (S) and 8 or 9 , obtained by placing $S$ at various points (and with different orientations) adjacent to B on the underside of DBA (fig. 13). The salient results are displayed in Table 14 for the cases $S=n$-pentane, $S=$ acetonitrile $(\mathrm{MeCN}), \mathrm{S}=$ benzonitrile $(\phi \mathrm{CN})$, and also for the case where $\mathrm{S}$ is absent. In all cases, the results for a given DBA/S pair correspond to a Boltzmannweighted rms-average of $T_{\text {if }}$ over the set of solvent structures sampled.

As expected on symmetry-grounds, $\left|T_{\text {if }}^{C S}\right|<<\left|T_{\text {if }}^{C R}\right|$ is generally found (the symmetry-breaking influence of solvent yields non-zero values for $\mathrm{T}_{\text {if }}^{\mathrm{CS}}$ ). The primary mechanistic question is the precise role of this symmetry-breaking in enhancing superexchange, as a function of solvent and bridge type. We see that solvent has only a modest (CS) or essentially no (CR) effect on coupling in the system with the "straight" bridge (8), whereas there is a strong solvent effect for both CS and CR in the system with the bent ("C-clamp") bridge (9), especially for the solvent with the lowest-lying empty orbitals $(\phi \mathrm{CN})$. It is also found (in results not shown) that removal of the central portion of the bridge ("annealing" the dangling bonds so created with $\mathrm{H}$ atoms) causes a drastic reduction of coupling strength in 8, while a similar treatment of 9 has very little effect [104].

We are thus led to the following picture. In 8 , the se coupling is dominated by the bridge (B), and this B-mediated coupling is indirectly affected to a modest 
degree by the solvent-induced symmetry-breaking of the zeroth-order $\mathrm{C}_{\mathrm{S}}$ wavefunctions (the solvent perturbs the molecular structure of the DBA moiety very little from $\mathrm{C}_{\mathrm{s}}$ geometry). In contrast, a solvent molecule within the $\mathrm{C}$-clamp cavity in 9 creates a dominant solvent-mediated superexchange pathway (DSA). The accessible orbitals of $\phi C N$ make it especially effective in this role, but even the saturated pentane molecule proves effective. These results underscore the point made above - namely, that covalent bonding is not a requirement for effective superchange coupling (Section 5.2.1). Finally, we note the good agreement between the calculated $T_{\text {if }}$ values and the values available from analysis of experimental kinetic data [106].

\subsubsection{Effective et Distances}

As a final illustration of the power of the GMH model, we evaluate the effective et distances (rDA, based on eqs 49 and 51 ) associated with the processes discussed in Section 5.4.1 and 5.4.2, and also for two mixed-valence metal-ion complexes, comparing the results with estimates $\left(\mathrm{r}_{\mathrm{DA}}^{0}\right)$ based on molecular structure. The results are summarized in Table 15 . For $\mathrm{Zn}_{2}\left(\mathrm{OH}_{2}\right)^{+}$, the rDA are all within $10 \%$ of the obvious candidate for $\mathrm{r}_{\mathrm{DA}}^{0}, \mathrm{rZnZn}^{\prime}$. The choice of $\mathrm{r}_{\mathrm{DA}}^{0}$ in the case of the larger $\mathrm{D}$ and $A$ groups in 8 and 9 is not so obvious. It turns out that the GMH values for both the CS and CR processes are close to the respective separation distances of the anthracene and double-bond midpoints. In contrast, for the two binuclear Ru complexes, the rDA values (obtained entirely in terms of experimental dipole and energy quantities $[75,76])$, are greatly reduced relative to $\mathrm{r}_{\mathrm{RuRu}}$ values, reflecting the role of strong ligand-field mixing in determining the centroids of the effective $D$ and $\mathrm{A}$ orbitals. 


\section{Concluding Remarks}

Quantitative evaluation and analysis of long-range electronic coupling involving many classes of donor-acceptor pairs and mediated by a wide range of intervening bridge types has become a major activity in theoretical chemistry. The techniques of computational molecular quantum mechanics in conjunction with orbital-based theories of electronic coupling, including particularly, the powerful hierarchy of superexchange models, have achieved major success in identifying in precise form the fundamental molecular principles controlling the coupling pertinent to long-range electron (and hole) transfer. Fine-tuning of system characteristics e.g., by variation of chemical composition of the primary sites (DBA) or the surrounding medium, or applying a variable external field - provides the basis for rational system design which is crucial to the emerging field of molecular electronics.

In the present chapter we have discussed general principles and theories (Sections 2-4) and then offered a number of specific examples of applications, mostly illustrating the properties of the important class of bridging materials comprising electronically saturated species. While the decomposition of bridges into local bonds and antibonds or similar "fine-grained" components will continue to serve as a rich source of insight into coupling through complex chemical systems, practical considerations for important cases of et in very large, extended systems pose a major challenge for the future - namely, to synthesize from the fine-grained ingredients, the more course-grained elements needed in the formulation of effective pathways suitable for manageable treatment of the very large systems. Electron transfer in protein systems is already playing an active role in this endeavor, driven by the goal of elucidating biochemical mechanisms, and major attention is being focussed on et in a number of other extended systems, 
$[1,15]$. The rapid growth in computer power and the development of sophisticated numerical techniques to exploit this power, will play an essential role by permitting exact solutions for suitable reference systems characterized by very large-scale model molecular Hamiltonians. Efforts toward design of materials with specified performance characteristics should also be greatly facilitated by development of tools for sensitivity analysis, permitting an assessment of whether charge transport is strongly controlled by a relatively small set of localized regions in an extended system, as opposed to more highly-distributed mechanisms.

\section{Acknowledgment}

We wish to acknowlege helpful discussions with Professor M.B. Zimmt (Brown University) and Dr. Y-P. Liu (Columbia University). This research was carried out at Brookhaven National Laboratory under contract DE-AC02-76CH00016 with the U.S. Department of Energy and supported by its Division of Chemical Sciences, Office of Basic Energy Sciences. 
Table 1. Coupling Through Linked Staffane Bridges $(2 \pi(\mathrm{m}) \text { and } 2 \sigma(\mathrm{m}))^{\mathrm{a}}$

\begin{tabular}{llllll}
\hline D/A Type & $\mathrm{m}$ & \multicolumn{2}{c}{ Radical Cations $\mathrm{b}, \mathrm{c}$} & \multicolumn{2}{c}{ Radical Anions $\mathrm{b,c}$} \\
\hline & & $-\mathrm{T}_{\mathrm{if}}^{\mathrm{KT}}$ & $\beta_{\mathrm{mm}+1}$ & $-\mathrm{T}_{\mathrm{if}}^{\mathrm{KT}}$ & $\beta_{\mathrm{mm}+1}$ \\
$\pi$ & 1 & 20.6 & 0.67 & 19.0 & 0.85 \\
& 2 & 6.52 & 0.66 & 4.35 & 0.84 \\
& 3 & 2.14 & - & 1.00 & - \\
$\sigma$ & 2 & -38.9 & 0.56 & -20.0 & 1.24 \\
& 3 & 15.6 & - & 2.63 & - \\
\hline
\end{tabular}

aSee Fig. 8a. Based on energy splitting (eq. (14)) at the KT level, using 3-21G orbital basis. The $\mathrm{m}=1 \sigma$ case is not included since it is dominated by $t s$ coupling. [After Table VI of [43], with permission.]

$\mathrm{b}_{\text {if }}$ given in mhartree ( 1 mhartree $=0.027 \mathrm{ev} \sim \mathrm{k}_{\mathrm{B}} \mathrm{T}$ at room temperature). The sign convention $[43,49]$ is based on the phases of the $D$ and $A$ orbitals in the two MO's of primarily D/A character and assigns a positive (negative) sign to the quantity $-\mathrm{T}_{\text {if }}$ when the occupation of the "in-phase" ("out-of-phase") MO is energetically favored (the positive sign for - $T_{\text {if }}$ corresponds to the "normal" situation in which in-phase ("bonding") orbital interactions are characterized by a negative transfer integral). For $2 \pi(\mathrm{m})$, the in-phase MO's are taken as those transforming as $a_{1}\left(C_{2 v}\right)$ or $b_{u}\left(C_{2 h}\right)$, while for $2 \sigma(m)$, "in phase" corresponds to $a_{1}\left(C_{2 v}\right)$ or $a_{g}\left(C_{2 h}\right)$.

cLocal $\beta\left(\AA^{-1}\right)$ defined analogously to eq (11): $\beta_{\mathrm{mm}+1}=2 \ln \left|\mathrm{T}_{\mathrm{if}}(\mathrm{m}+1) / \mathrm{T}_{\mathrm{if}}(\mathrm{m})\right| / \Delta \mathrm{r}$, where $\Delta r=3.37 \AA$ in the mean axial "length" of a staffane unit (see [43]). 
Table 2. Distance-dependence of Coupling through Non-covalently-linked Bridge Systems ${ }^{\mathbf{a}}$

\begin{tabular}{llll}
\hline Bridge Unit & $\mathrm{m}$ & Radical Cation & Radical Anion \\
\hline & & $\beta_{\mathrm{mm}+1}^{\mathrm{b}}$ & $\beta_{\mathrm{mm}+1}^{\mathrm{b}}$ \\
$\mathrm{CH}_{4}(\mathbf{4} \sigma(\mathrm{m}))$ & 1 & 0.94 & 0.84 \\
& 2 & 1.01 & 0.86 \\
& & & \\
$\mathrm{H}_{2} \mathrm{O}^{\mathrm{c}}$ & 1 & 0.96 & \\
& 2 & 0.94 & \\
\hline
\end{tabular}

aBased in part on Table $\mathrm{X}$ of [43].

bSee footnote c of Table $1 ; \Delta r=3.4$ and $2.8 \AA$, respectively, for $\mathrm{CH}_{4}$ and $\mathrm{H}_{2} \mathrm{O}$.

'Analogous to the $4 \sigma(\mathrm{m})$ structures, but with $\mathrm{CH}_{4}$ 's replaced by $\mathrm{H}_{2} \mathrm{O}$ 's. The orientation of the $\mathrm{H}_{2} \mathrm{O}$ 's corresponds to the $\mathrm{CH}_{2}$ moieties of the $\mathrm{CH}_{4}$ units in $4 \sigma(\mathrm{m})$ whose planes are perpendicular to the $\mathrm{D} / \mathrm{A}$ axis. Van der waals $\mathrm{C} \cdots \mathrm{O}$ contacts of $3.1 \AA$ were assigned. 
Table 3. Conformational Dependence of $\mathrm{T}_{\text {if }}$ for $\left(\mathrm{CH}_{2}\right)_{6}^{ \pm}$Radical Ions $(1 \pi(3))^{\mathrm{a}}$

\begin{tabular}{llll}
\hline & & $\begin{array}{l}\text { Radical Cation } \\
-T_{\text {if }}\end{array}$ & $\begin{array}{l}\text { Radical Anion } \\
-T_{\text {if }}\end{array}$ \\
\hline $\begin{array}{l}\text { Conformation of } \mathrm{D} \\
\text { and A orbitals } \\
\text { relative to bridge } \mathrm{b}\end{array}$ & $\begin{array}{l}\text { Conformation of } \\
\mathrm{C}_{4} \text { bridge }\end{array}$ & & \\
in-plane & & & \\
& trans $\left(\mathrm{C}_{2 \mathrm{~h}}\right)$ & -11.0 & -8.4 \\
& $\begin{array}{l}\text { gauche }\left(\mathrm{C}_{2}\right) \\
\text { cis }\left(\mathrm{C}_{2 \mathrm{v}}\right)\end{array}$ & -2.4 & -4.5 \\
& & & -4.3 \\
perpendicular to & & & \\
plane & & & \\
& trans $\left(\mathrm{C}_{2 \mathrm{~h}}\right)$ & -1.1 & \\
& gauche $\left(\mathrm{C}_{2}\right)$ & +3.6 & +0.3 \\
cis $\left(\mathrm{C}_{2 \mathrm{v}}\right)$ & +7.9 & +2.0 \\
\hline
\end{tabular}

aAfter Table VI of [49]. Sign convention for $T_{\text {if }}$ (mhartree) as defined in footnote $b$ of Table 1. For the $\left(\mathrm{CH}_{2}\right)_{6}^{ \pm}$systems, "in-phase" is taken as transforming according to $\mathrm{a}_{\mathrm{g}}$, a, and $\mathrm{a}_{1}$ for, respectively, $\mathrm{C}_{2 \mathrm{~h}}, \mathrm{C}_{2}$, and $\mathrm{C}_{2 \mathrm{v}}$ symmetry with "in plane" D/A conformers, and $a_{u}, a$, and $b_{1}$ for the corresponding "perpendicular" conformers .

bAngle of the $D(A)$ pi orbitals relative to plane formed by the carbon atom of the terminal $D(A)$ methylene group and the closest two carbon atoms of the bridge. 
Table 4. Conformational Dependence of $\left|T_{i f}\right|$ for Polycyclic Bridge Units ${ }^{a}$

\begin{tabular}{lll}
\hline DBA & \multicolumn{2}{c}{ T $_{\text {Tif }}$ ecl/T } \\
& Radical Cation & Radical Anion \\
$3 \pi(2)$ & 1.6 & $\geq 10^{\mathrm{b}}$ \\
$2 \pi(2), 2 \sigma(2)$, & $1.0-1.1$ & $1.0-1.2$ \\
$3 \sigma(2)$ & & \\
\hline
\end{tabular}

aAfter Table VIII of [43], based on systems with 2 bridge units $(2 \pi(2), 2 \sigma(2), 3 \pi(2)$, and $3 \sigma(2)$ ). Staggered ("stagg") and eclipsed ("ecl") conformers correspond, respectively, to the $C_{2 h}$ and $D_{3 d} m=2$ structures as drawn (fig. 8), and to the $C_{2 v}$ and $D_{3 h}$ variants obtained by rotating one bridge unit $60^{\circ}$ relative to the other.

bInequality is due to sensitivity of calculated $T_{\text {if }}$ value for the staggered conformer [43]. 
Table 5. Comparison of coupling through three-bond linksa: Covalent bonds vs. hydrogen bonds ${ }^{b}$

\begin{tabular}{|c|c|c|c|}
\hline DBA & $\begin{array}{l}\mathrm{D} / \mathrm{A} \\
\text { Conformation }^{\mathrm{c}}\end{array}$ & $\begin{array}{l}-T_{\text {if }}(\text { mhartree })^{d} \\
\text { Radical Cation }\end{array}$ & Radical Anion \\
\hline $\begin{array}{l}\text { Covalent links } \\
\left(\mathrm{rDA}_{\mathrm{DA}}=5.8 \AA\right) \\
5 \pi\end{array}$ & $\begin{array}{l}\text { "in plane" } \\
\text { "out of plane" }\end{array}$ & $\begin{array}{l}-0.7 \\
+2.6\end{array}$ & $\begin{array}{l}-9.1 \\
+4.3\end{array}$ \\
\hline $\begin{array}{l}\text { H-bonded links } \\
(\text { rDA }=7.0 \AA) \\
6 \pi\end{array}$ & $\begin{array}{l}\text { "in plane" } \\
\text { "out of plane" }\end{array}$ & $\begin{array}{l}-4.2 \\
+0.5\end{array}$ & $\begin{array}{l}-4.2 \\
+0.6\end{array}$ \\
\hline
\end{tabular}

aThe single bonds linking the terminal $\mathrm{CH}_{2}$ groups to the bridge are not counted in the total number of linker bonds since they are very weakly coupled to the $\mathrm{D}$ and $\mathrm{A}$ orbitals (i.e., the $2 \mathrm{p} \pi$-type orbitals) [43].

bResults based on the 3-21G orbital basis set, at the KT level (eq. 14)).

cThe "conformation" of the $\mathrm{D}(\mathrm{A})$ orbital is defined by the dihedral angles between the plane perpendicular to the $\mathrm{D}(\mathrm{A}) \mathrm{CH}_{2}$ group and the plane defined by the $\mathrm{D}(\mathrm{A})$ carbon atom and the closest two atoms in each of the two linkers it is attached to. By this criterion, $5 \pi$ and $6 \pi$ as drawn correspond, respectively, to dihedral angles $60^{\circ}$ and $90^{\circ}$, and are designated as "out of plane." The "in-plane" conformers, obtained by $90^{\circ}$ rotations of the $\mathrm{D}$ and A groups, correspond, respectively, to dihedral angles of $30^{\circ}$ and $0^{\circ}$.

dSign convention as described in footnote b of Table 1 . In $\mathrm{C}_{2 \mathrm{v}}$ symmetry $(5 \pi)$, "inphase" is taken to correspond to the $a_{1}$ (as drawn) or $b_{1}$ ("in-plane" conformer) representations, whereas for $\mathrm{C}_{2 \mathrm{~h}}$ symmetry $(6 \pi)$, the corresponding representations are $a_{u}$ (as drawn) or $a_{g}$.

eThe hydrogen-bonded $0 \cdots O$ separation is $3.0 \AA$. 
Table 6. Effect of peripheral aqueous solvent on coupling through trans-alkyl bridges $(1 \pi(\mathrm{m}))^{\mathbf{a}}$

Nature of Hydration Decay Coefficient, $\beta\left(\AA^{-1}\right)^{b}$

Radical Cations Radical Anions

Solvent-free ${ }^{c}$

0.85

0.95

Dielectric

0.85

0.95

continuum ${ }^{d}$

$\begin{array}{lll}\text { Specific hydration } & 0.81 & 0.95\end{array}$

$\left(\left(\mathrm{CH}_{2}\right)_{\mathrm{m}+3}\left(\mathrm{H}_{2} \mathrm{O}\right)_{\mathrm{m}+1}\right)^{ \pm}$

aBased on SCF energy splittings (eq. (14)), with the $6-31 \mathrm{G}^{* *}$ orbital basis, and including $\mathrm{m}$ in the range $2-6$ (see [52]).

bEq. (11), based on a linear least-squares fit of $\ln \left|T_{\text {if }}\right|$ as a function of rDA. The $\beta$ values are somewhat larger than those obtained with the $3-21 \mathrm{G}$ basis ("SCF/deloc" results displayed in fig 7 ).

In vacuo results from [52].

dBased on dielectric cavity model described in[ 52].

eAn $\mathrm{H}_{2} \mathrm{O}$ interacts with each of $\mathrm{m}+1 \mathrm{CH}_{2}$ triads for each $\left(\mathrm{CH}_{2}\right)_{\mathrm{m}+3}$ species, oriented so as to allow the $\mathrm{H}_{2} \mathrm{O}$ lone pairs to overlap optimally with the carbon framework (as indicated in 7), with van der waals $\mathrm{C} \cdots \mathrm{O}$ contacts of $3.1 \AA$. 
Table 7. Gaps $\left(\left|\Delta_{j}\right|\right)$ for Trans-Staggered Alkane Bridge Systems $(1 \pi(\mathrm{m}))$ in a Local (NBO) Basis ${ }^{\mathbf{a}, \mathbf{b}}$

\begin{tabular}{lr}
\hline bond type & \multicolumn{1}{c}{$\Delta \mathrm{j}$} \\
\hline $\mathrm{CC}$ & $10.9-12.2$ \\
$\mathrm{CH}$ & $8.2-8.4$ \\
$\mathrm{CC}^{*}$ & $8.7-10.1$ \\
$\mathrm{CH}^{*}$ & $11.4-11.7$ \\
\hline
\end{tabular}

aThe indicated quantities represent the magnitude (in $\mathrm{eV}$ ) of the lowest-energy gap $\left(\Delta_{j}\right)$ between D/A orbitals and the highest-lying occupied NBO of the indicated bond type ( $\mathrm{CC}$ or $\mathrm{CH}$ ) for the radical cation systems, and the lowest-lying unoccupied $\mathrm{NBO}$ of the given anti-bond type $\left(\mathrm{CC}^{*}\right.$ or $\left.\mathrm{CH}^{*}\right)$ for the radical anion systems. bThe orbital gaps are based on the diagonal elements of the Fock matrix in the NBO basis, corresponding to $\alpha$-spin (for the radical cations) and $\beta$-spin (for the radical anion) manifolds of the neutral diradical parent (treated at the UHF level with the 3-21G basis) [43]. 
Table 8. Hopping Integrals for Trans-Staggered Alkane Bridge Systems $(1 \pi(\mathrm{m}))$ in a Local (NBO) Basisa

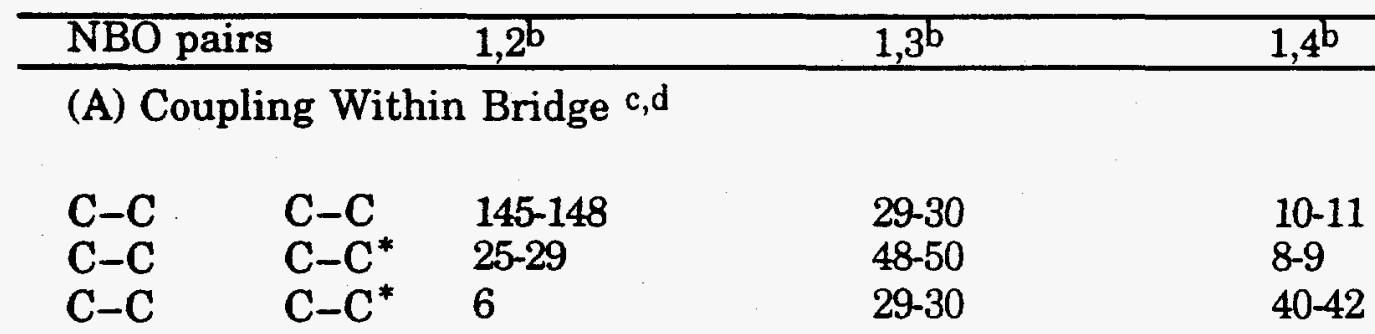

(B) D/A and CC or $\mathrm{CC}^{*}$ (Radical Cations)

$\begin{array}{lllll}\text { D/A } & \text { C-C } & 53 & 17-18 & 4 \\ \text { D/A } & \text { C-C } & 96 & 41 & 2 \\ \text { D/A } & \text { C-C } & & & \\ \text { D/A } & \text { C-C }^{*} & & & \end{array}$

(C) D/A and CC or $\mathrm{CC}^{*}$ (Radical Anions)

$\begin{array}{lllll}\text { D/A } & \text { C-C } & 79 & 23 & 7 \\ \text { D/A } & \text { C-C }^{*} & 76 & 41 & 2\end{array}$

aThe listed quantities are the magnitudes (in mhartrees) of the hopping integrals for the indicated NBO pairs, based on the Fock matrix [43] (see footnote 6 of Table 7).

b1,2 (nearest-neighbor), 1,3 (next-nearest-neighbor), and 1,4 denote successively longer-range NBO interactions (the NBO pairs involved in 1,2 interactions share a common $\mathrm{C}$ atom).

cThe values given for coupling between bridge NBO's apply to both radical cation and anion species.

dWhile the NBO Fock matrix is not in general diagonal with respect to a $\mathrm{CC} / \mathrm{CC}^{*}$ (or $\mathrm{CH} / \mathrm{CH}^{*}$ ) pair sharing a common set of atoms, the magnitude of such coupling is small ( $\leq 0.01$ hartree) [43]. 
Table 9. Through-Space ( $t s$ ), Hole (h), Electron (e), and Hybrid (h/e) Contributions (\%) to $\mathrm{T}_{\text {if }}$ from $\mathrm{CC}$ and $\mathrm{CC}^{*} \mathrm{NBO}^{\mathrm{a}} \mathrm{s}^{\mathrm{a}}$ for Odd-Membered Trans-Staggered Alkane Bridges $(\mathbf{1} \pi(\mathrm{m}))$

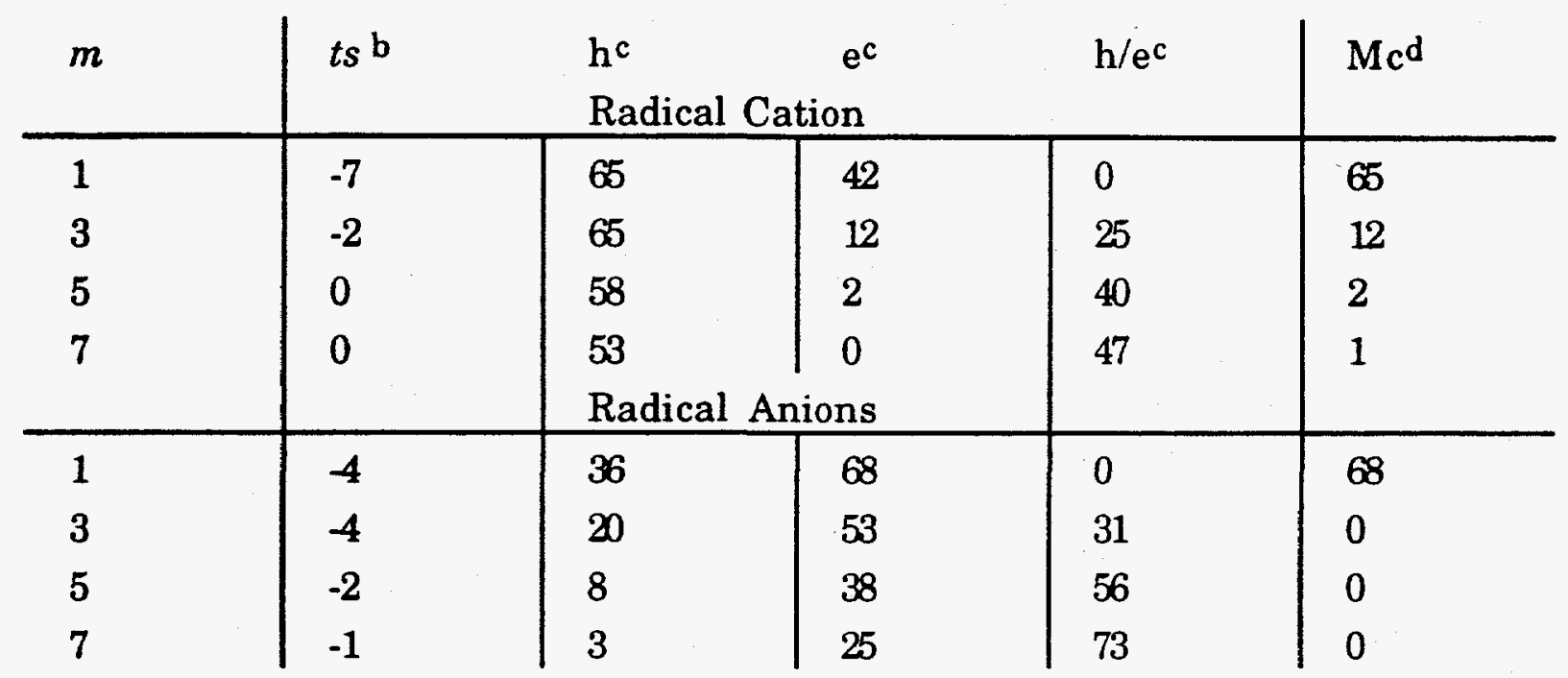

aThe perturbative estimate of $\mathrm{T}_{\text {if }}$ based on the $\mathrm{CC}$ and $\mathrm{CC}^{*} \mathrm{NBO}$ 's of the spacer group (terminal $\mathrm{CC}$ and $\mathrm{CC}^{*}$ bonds linking the $\mathrm{D} / \mathrm{A} \mathrm{CH}$ groups to the spacer are omitted); obtained with an STO-3G basis and reproduced with permission from Table IV of [43].

bThrough-space contribution (based on Fock matrix element between $\mathrm{D}$ and $\mathrm{A}$ NBO's). Since the $t s$ term differs in sign from the other contributions, it is assigned a negative percent contribution.

ch,e and h/e denote contributions based, respectively, on pathways of the hole, electron and hybrid (both hole and electron) type, as discussed in section 3.4. Together with the ts term, these contributions account for $100 \%$ of the $\mathrm{CC}$ and $\mathrm{CC}^{*}$ contributions to $T_{\text {if. }}$

dMc denotes the contributions from the nearest-neighbor (NN) pathway of the hole type (radical cations) or electron type (radical anions), as given by eq (31). 
Table 10. Renormalized McConnell-type (NN) Model for Coupling Through TransStaggered Alkane Bridges ${ }^{a}$

$\mathrm{T}_{\text {if }} \propto \mathrm{f}_{\mathrm{m}}\left(\mathrm{t}_{12} / \Delta\right)^{\mathrm{m}-1}$

\begin{tabular}{lll}
\hline $\mathrm{m}$ & & $\mathrm{f}_{\mathrm{m}}$ \\
\hline 2 & 1.00 & \\
4 & 2.44 & \\
6 & 6.27 & \\
8 & 16.10 & \\
\hline
\end{tabular}

a Based on results reported in Table 7 of [63], at the $3-21 \mathrm{G} / \mathrm{KT}$ and $6-31+\mathrm{G} / \mathrm{KT}$ levels for radical cations with bridges the same as for $1 \pi(\mathrm{m}), \mathrm{m}$ even, but with terminal vinyl $\mathrm{D}, \mathrm{A}$ groups $\left(\mathrm{t}_{12}\right.$ refers to $\mathrm{NN}$ coupling between $\mathrm{C}-\mathrm{C} \sigma \mathrm{NBO}$ 's and was assigned a typical value of $-4.0 \mathrm{eV}$, while the orbital energy gap $\Delta$ was taken as $10 \mathrm{eV})$. The "renomalization" coefficients $\mathrm{f}_{\mathrm{m}}$ were evaluated so as to reproduce the numerical results from perturbative calculations based on all possible "forward" pathways involving the C-C NBO's (i.e., all "forward" pathways of the pure "hole" type). 
Table 11. Corrrelation of Decay coefficient $(\beta)$ with $D / A$ energy level $E_{D / A}^{0}$ and gap $(\Delta)$ for trans-staggered $\left(\mathrm{X}\left(\mathrm{CH}_{2}\right)_{\mathrm{m}-1} \mathrm{X}\right)+$ radical cations ${ }^{\mathrm{a}}$

\begin{tabular}{lll}
$\mathrm{X}$ & $\mathrm{E}_{\mathrm{D} / \mathrm{A}^{0}(\Delta)^{\mathrm{c}}}^{\mathrm{b}}$ & $\beta^{\mathrm{d}}$ \\
\hline $\mathrm{H}_{2} \mathrm{~N}-$ & $-9.8(11.2)$ & 0.50 \\
$\mathrm{HS}-$ & $-10.1(11.7)$ & 0.43 \\
$\mathrm{H}_{2} \mathrm{C}=\mathrm{CH}-$ & $-10.1(10.8)$ & 0.41 \\
$\mathrm{HC} \equiv \mathrm{C}-$ & $-10.9(10.3)$ & 0.20 \\
$\mathrm{HO}$ & $-12.6(9.0)$ & 0.08
\end{tabular}

a Adapted from data given in [63]; energies in $\mathrm{eV}, \beta$ in $\AA^{-1}$ (based on $\Delta \mathrm{r}=1.27 \AA$ ), m is the number of CC sigma bonds (represented by NBO's based on the KT level with a 3-21G orbital basis.)

bLimiting values for $\mathrm{m}=14\left(\left(\mathrm{E}_{\mathrm{D} / \mathrm{A}}^{0}\right)\right.$ depends only weakly on $\left.\mathrm{m}\right)$.

c. Mean value of $\Delta_{j}^{h}$ over all internal CC bonds of bridge, based on $m=6$ results.

dLimiting results for large $\mathrm{m}\left(\beta_{12,14}\right.$; see definition of local $\beta$ given in footnote $c$ of Table 1). The local $\beta$ 's diminish in magnitude by up to a factor of two as $\mathrm{m}$ ranges from 2 to 16. 
Table 12. Additivity Relationships Among $T_{\text {if }}$ Values (mhartree) for Radical Anion Coupling Through cis 3-bond Bridge Linkersa

Number of Linkers D/A symmetry type

\begin{tabular}{ccl}
\hline & $\sigma$ & \multicolumn{1}{c}{$\pi$} \\
\hline 1 & $-19.8(-19.8)^{\mathrm{b}}$ & $-4.3(-4.3)$ \\
2 & $-37.9(-39.6)$ & $+4.1(-2.1)$ \\
3 & $-53.2(-59.4)$ & $-6.0(-6.5)$
\end{tabular}

a. The relevant DBA systems are schematically depicted in the last three entries of each column in fig. 11. The sign conventions for the $-T_{\text {if }}$ quantities are as given in footnote $b$ of Table 1 , with "in-phase" corresponding to $a_{1}\left(C_{2 v}\right)$ or $a_{1 g}\left(C_{2 h}\right)$. Results are taken from Table II of [49] and are NBO through bond $(t b)$ contributions for the radical anions treated at the $3-21 \mathrm{G} / \mathrm{KT}$ level.

b. The quantities in parentheses are the expected values based on simple additive contributions from each linker, where each linker is assumed to follow an effective McConnell relationship proportional to $\mathrm{T}^{2}$ (see eq 31 ), and $\mathrm{T}$ is weighted by an appropriate direction cosine in the case of $\pi$-type $D / A$ groups. 
Table 13. Values of $\left|T_{\text {if }}\right|$ (mhartree) and Decay Coefficients, $\beta\left(\AA^{-1)}\right.$ for Four et Processes in $\mathrm{Zn}_{2}\left(\mathrm{OH}_{2}\right)^{+\mathbf{a}}$

\begin{tabular}{lc}
$\begin{array}{l}\text { et } \\
\text { process }\end{array}$ & $\mathrm{rZnO}(\AA)$ \\
\hline & 2.05
\end{tabular}

A) $\left|\mathrm{T}_{\text {if }}\right|$ for $\mathrm{ZZnZn}^{\prime}=5.0 \AA$

$\mathbf{s} / \mathbf{s}^{\prime}$

$\mathrm{p} / \mathrm{p}^{\prime}$

$s / p^{\prime}$

$\mathrm{p} / \mathbf{s}^{\prime}$

B) $\beta$ based on range $\mathrm{r} \mathrm{Zn}_{\mathrm{Zn}} \mathrm{Zn}^{\prime}=5.0 \cdot 9.0 \AA^{\mathrm{c}}$

$\mathbf{s} / \mathbf{s}^{\prime}$

$\mathrm{p} / \mathbf{p}^{\prime}$

$\mathbf{s} / \mathbf{p}^{\prime}$

$\mathrm{p} / \mathbf{s}^{\prime}$
8.8

10.4

30.8

21.9
7.8

14.3

35.9

22.2
7.1

13.0

24.4

24.4

a See structure in fig 12 and description of calculations in caption.

b The four et processes are labelled in terms of the dominant valence orbitals involved in each case (see configurations 57a-d), based on the diabatic states determined by the GMH analysis [77].

'Linear regression coefficient $r \geq 0.99$ in least squares fits of $\ln \left|\mathrm{T}_{\mathrm{if}}\right|^{2}$ vs $\mathrm{rZnZn}$ '. 
Table 14. Solvent Effect on $\left|T_{\text {if }}\right|\left(\mathrm{cm}^{-1}\right)^{\mathrm{a}}$ for systems 8 and 9

(9)

\begin{tabular}{|c|c|c|c|c|}
\hline Solvent & 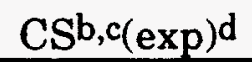 & CRe & $\mathrm{CS}^{\mathrm{b}, \mathrm{c}(\exp )}$ & $\mathrm{CR}^{\mathrm{e}}$ \\
\hline Solvent-free & $4-$ & 68 & $0.1-$ & 3 \\
\hline n-pentane & $4-$ & 70 & $16-$ & 60 \\
\hline $\mathrm{MeCN}$ & $6 \quad(15)$ & 62 & $7 \quad(21)$ & 16 \\
\hline$\phi C N$ & $19(12)$ & 72 & $46 \quad(65)$ & 231 \\
\hline & \multicolumn{2}{|c|}{$\begin{array}{l}\text { "indirect solvent effect } \\
\text { (dominant DBA } \\
\text { coupling) }\end{array}$} & \multicolumn{2}{|c|}{$\begin{array}{l}\text { "direct" solvent effect } \\
\text { (dominant DSA } \\
\text { coupling) }\end{array}$} \\
\hline
\end{tabular}

a) Units of $\mathrm{cm}^{-1}\left(8066 \mathrm{~cm}^{-1}=1 \mathrm{eV}\right)$ are used here (in contrast to the use elsewhere of mhartree) to accommodate the very small magnitude of some of the $T_{\text {if }}$ values. Results based on GMH diabatic states obtained from INDO/S CI calculations [102].

b) $\mathrm{CS} \equiv$ charge separation (see fig $3 \mathrm{~b}$ ): $\mathrm{D}^{*} \mathrm{~A} \rightarrow \mathrm{D}^{+} \mathrm{A}^{-}$

c) symmetry-forbidden for $\mathrm{C}_{\mathrm{s}}$ point-group symmetry (see fig 13 ).

d) Experimental values [104]

e) $\mathrm{CR} \equiv$ charge recombination (see fig $3 b$ ): $\mathrm{D}^{+} \mathrm{A}^{-} \rightarrow \mathrm{DA}$. 
Table 15. Effective Separation of D/A Sites, $\mathrm{rDA}_{\mathrm{DA}}(\AA)$

et Process

$$
\begin{aligned}
& \text { From Molecular } \\
& \text { Geometry } \\
& \left(\mathrm{r}_{\mathrm{DA}}^{0}\right)
\end{aligned}
$$

A) $\mathrm{Zn}_{2}\left(\mathrm{H}_{2} \mathrm{O}\right)+\mathrm{b}$

$\mathrm{s} / \mathrm{s}^{\prime}$
$\mathrm{s} / \mathrm{p}^{\prime}$
$\mathrm{p} / \mathbf{s}^{\prime}$
$\mathrm{p} / \mathrm{p}^{\prime}$

B) 8
$\mathrm{CS}^{\mathrm{c}}$
$11.6^{\mathrm{d}}$
$12.1^{\mathrm{e}}$
$\mathrm{CR}^{\mathrm{f}}$
$11.6^{\mathrm{d}}$
$11.8^{\mathrm{e}}$

C) 9

$\begin{array}{lll}\mathrm{CS}^{\mathrm{c}} & 7.1^{\mathrm{d}} & 7.2^{\mathrm{e}} \\ \mathrm{CR}^{\mathrm{f}} & 7.1^{\mathrm{d}} & 7.0^{\mathrm{e}}\end{array}$

D) $\left(\mathrm{Ru}^{2+} \mathrm{L} \mathrm{Ru}^{3+}\right)$
ITg $\left\{\begin{array}{l}\mathrm{L}=\mathrm{pz} \\ \mathrm{L}=\mathrm{bpy}\end{array}\right.$
$6.8^{\mathrm{h}}$
$1.4^{\mathrm{i}}$
$11.3^{\mathrm{h}}$
$5.2^{\mathrm{i}}$

a See Section 4 and [77].

bFrom 4-state GMH analysis, using results of CASSCF calculations (Section 5.4

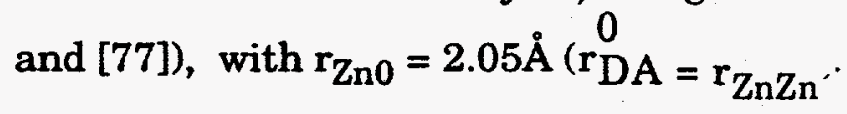

cCharge separation process (see fig $3 b$ ).

d Distance separating the midpoints of the anthracene (D) and olefinic $\mathrm{C}=\mathrm{C}$ bond $(\AA)$.

eBased on 3-state GMH analysis, using results from INDO/S CI calculations [104] 
fCharge recombination process (see fig $3 b$ )

gIntervalence Transfer (IT), the optical et process depicted in fig 3a; pz and bpy denote the pyrazine and 4,4-bipyridine ligands. Each $\mathrm{Ru}$ also has five peripheral $\mathrm{NH}_{3}$ ligands.

$\mathrm{h}_{\mathbf{r}_{\mathrm{DA}}}^{0}=\mathrm{rRuRu}_{\mathrm{RuR}}$

iFrom 2-state GMH analysis using experimental adiabatic data(transition energy [76], dipole moment shift [75], and transistion dipole [76]). 


\section{References}

1 Mikkelsen KV, Ratner MA. Chem Rev 1987; 87: 113.

2 Marcus RA, Sutin N. Biochim Biophys Acta 1985; 811: 265.

3 Newton MD, Sutin N. Ann Rev Phys Chem 1984; 35: 437.

4 Chem Rev 1992; 92: No 3 (entire issue).

5 Bertrand P. Structure and Bonding 1991; 75: 1.

6 Moser CC, Keske JM, Warncke K, Farid RS, Dutton PL. Nature 1992; 355: 796.

7 Sumi H, Marcus RA. J Chem Phys 1986; 84: 4894.

8 Bader JS, Kuharski RA, Chandler D. J Chem Phys 1990; 93: 230.

9 Kim HJ, Hynes JT. J Chem Phys 1992; 96: 5088.

10 Jortner J, Bixon M. J Chem Phys 1988; 88: 167.

11 Larsson S. J Am Chem Soc 1981; 103: 4034.

12 Ratner MA. J Phys Chem 1990; 94: 4877.

13 Onuchic JN, Beratan DN. J Chem Phys 1990; 92: 722.

14 Newton MD. Chem Rev 1991; 91: 767.

15 Mirkin CA, Ratner, MA. Ann Rev Phys Chem 1992; 43: 719.

16 Aviram A, ed. In: Molecular Electronics--Science and Technology. New York: Am Inst Phys, 1992.

17 Aviram A, Ratner MA. Chem Phys Lett 1974; 29: 277.

18 Aviram A. J Am Chem Soc 1988; 110: 5687.

19 Kemp M, Mujica V, Ratner MA. J Chem Phys 1994; 101: 5172.

20 Mujica V, Kemp M, Ratner, MA. J Chem Phys 1994; 101: 6856.

21 Petrov EG, Tolokh IS, Demidenko AA, Gorbach VV. Chem Phys 1995; 193 : 237.

22 Joachim C. New J Chem 1991; 15: 223.

23 Waldeck DH, Beratan DN. Science 1993; 261: 576.

24 Farazdel A, Dupuis M. Phys Rev 1991; B44: 3909. 
25 Hush NS, Wong AT, Bacskay GB, Reimers JR. J Am Chem Soc 1990; 112: 4192.

26 Reimers JR, Hush NS. Chem Phys 1993; 176: 407.

27 Broo A, Zerner MC. Chem Phys 1995; 196: 423.

28 Cave RJ, Baxter DV, Goddard III WA, Baldeschwieler JD: J Chem Phys 1987; 87: 926.

29 Warshel A., Creighton S, Parson WW. J Phys Chem 1988; 92: 2696.

30 Scherer POJ, Fischer SF. Chem Phys 1989; 131: 115.

31 Marchi M, Gehlen JN, Chandler D, Newton MD. J Am Chem Soc 1993; 115: 4178.

32 Marcus RA. Chem Phys Lett 1987; 133: 471.

33 Reimers JR, Hush NS. Chem Phys 1990; 146: 105.

34 Jordan KD, Paddon-Row MN. Chem Rev 1992; 92: 395.

35 Michel-Beyerle ME, Bixon M, Jortner J. Chem Phys Lett 1988; 151: 188.

36. Koga N, Sameshima K, Morokuma K. J Phys Chem 1993; 97: 13117.

37. Scholes GD, Ghiggino KP, Oliver AM, Paddon-Row MN. J Phys Chem 1993; 97: 11871.

38 Onuchic JN, Beratan DN, Hopfield JJ. J Phys Chem 1986; 90: 3707.

39 Tang J. J Chem Phys 1993; 98: 6263.

40 Landau L. Phys Z Sowjet 1932; 2: 46; Zener C. Proc Roy Soc London1932; Ser A: 696.

41 Kuznetsov AM, Ulstrup J. J Chem Phys 1981; 75: 2047.

42 Beratan DN, Onuchic JN, Hopfield JJ. J Chem Phys 1987; 86: 4488.

43 Liang C, Newton MD. J Phys Chem 1993; 97: 3199.

44 Stuchebrukhov AA. Chem Phys Lett 1994; 225: 55.

45 Newton MD, Ohta K, Zhong E. J Phys Chem 1991; 95: 2317.

46 Gamow G. Z Phys 1928; 51: 204.

47 McConnell HM, ed. J Chem Phys 1961; 35: 508.

48 Miller JR, Beitz JV. J Chem Phys 1981; 74: 6746.

49 Liang C, Newton MD. J Phys Chem 1992; 96: 2855. 
50 Siddarth P, Marcus RA. J Phys Chem 1992; 96: 3213.

51 Arnold BR, Noukakis D, Farid S, Goodman JL, Gould IR. J Am Chem Soc 1995; 117: 4399.

52 Liu Y-P, Newton MD. J Phys Chem 1995; 99: 12382.

53 Evenson JW, Karplus M. J Chem Phys 1992; 96: 5272.

54 Skourtis SS, Beratan DN, Onuchic JN. Chem Phys 1993; 176: 501.

55 Wynne K, Galli C, Hochstrasser RM. J Chem Phys 1994; 100: 4797.

56 Todd MD, Nitzan A, Ratner MA. J Phys Chem 1993; 97: 29.

57 Beratan DN, Onuchic JN. ACS Advances in Chemistry Series 1991; 228: 71.

58 Onuchic JN, de Andrade PCP. J Chem Phys 1991; 95: 1131.

59 Gruschus JM, Kuki A. J Phys Chem 1993; 97: 5581.

60 Okada A, Kakitani T, Inoue J. J Phys Chem 1995; 99: 2946.

61 Stuchebrukhov AA, Marcus RA. J Phys Chem 1995; 99: 7581.

62 Regan JJ, Risser SM, Beratan DN, Onuchic JN. J Phys Chem 1993; 97: 13083.

63 Shephard MJ, Paddon-Row MN, Jordan KD. Chem Phys 1993; 176: 289.

64 Curtis LA, Naleway CA, Miller JR. Chem Phys 1993; 176: 387.

65 Beratan DN, Betts JN, Onuchic JN. J Phys Chem 1992; 96: 2852.

66 Skourtis SS, Regan JJ, Onuchic JN. J Phys Chem 1994; 98: 3379.

67 Arnobio A, da Gama S. J Theor Biol 1990; 142: 251.

68 Goldman C. Phys Rev A 1991; 43: 4500.

69 Lopez-Castillo J-M, Filali-Mouhim A, Jay-Gerin J-P. J Phys Chem 1993; 97 : 9266.

70 Evenson JW, Karplus M. Science 1993; 262: 1247.

71 Richardson DE, Taube H. J Am Chem Soc 1983; 105: 40.

72 Bixon M, Jortner J, Verhoeven JW. J Am Chem Soc 1994; 116: 7349.

73 Mulliken RS. J Am Chem Soc 1952; 64: 811.

74 Hush NS. Electrochim Acta 1968; 13: 1005.

75 Reimers JR, Hush NS. J Phys Chem 1991; 95: 9773. 
76 Creutz C, Newton MD, Sutin N. J Photochem Photobiol A: Chem 1994; 82: 47.

77 Cave RJ, Newton MD. Chem Phys Lett 1996 (in press).

78 Sutin N. ACS Advances in Chemistry Series 1991; 228: 25.

79 Werner H-J, Meyer W. J Chem Phys 1981; 74: 5802.

80 Macias A, Riera A. J Phys 1978; B11: L489.

81 Kato S, Amatatsu Y. J Chem Phys 1990; 92: 7241.

82 Kim HJ, Bianco R, Gertner BJ, Hynes, JT. J Phys Chem 1993; 97: 1723.

83 Shin Y-GK, Brunschwig BS, Creutz C, Sutin N. J Am Chem Soc 1995; 117: 8668.

84 Cave RJ, Newton MD; to be published.

85 Pacher T, Cederbaum LS, Köppel H. J Chem Phys 1988; 89: 7367.

86 Domcke W., Woywood C, Sengle M. Chem Phys Lett 1994; 226: 257.

87 Marchi M, Chandler D. J Chem Phys 1991; 95: 889.

88 Braga M, Larsson S. Ghem Phys Lett 1993; 213: 217.

89 Kim K, Jordan K.D, Paddon-Row MN. J Phys Chem 1994; 98: 11053.

90 Newton MD. $J$ Phys Chem 1991; 95: 30.

91 Zerner MC, Loew GH, Kirchner RF, Mueller-Westerhoff UT. J Am Chem Soc 1980; 102: 589.

92 Koopmans T. Physica 1993; 1: 104.

93. Braga M, Larsson S. J Phys Chem 1993; 97: 8929.

94 Curtiss LA, Naleway CA, Miller JR. J Phys Chem 1995; 99: 1182.

95 Curtiss LA, Naleway CA, Miller JR. J Phys Chem 1993; 97: 4050.

96 Krongauz VV. J Phys Chem 1992; 96: 2609.

97 Naleway CA, Curtiss LA, Miller JR. J Phys Chem 1991; 95: 8434.

98 Liu YP, Newton MD; unpublished work.

99 Reed AE, Curtiss LA, Weinhold F. Chem Rev 1988; 88: 899.

100 Shephard MJ, Paddon-Row MN, Jordan KD. J Am Chem Soc 1994; 116: 5328.

101 Beratan DN. J Am Chem Soc 1986; 108: 4321. 
102 Onuchic JN, Beratan DN. J Am Chem Soc 1987; 109: 6771.

103 Reimers JR, Hush NS. Inorg Chim Acta 1994; 226: 33.

104 Cave RJ, Newton MD, Kumar K, Zimmt MB $J$ Phys Chem (in press).

105 Allinger NL. J Amer Chem Soc 1977; 99: 8127.

106 Kumar K, Sin Z, Waldeck DH, Zimmt MB. J Am Chem Soc (in press). 


\section{Figure Captions}

Figure 1. Generic electron transfer system, $\mathrm{DBA}^{+} \mathrm{D}^{+} \mathrm{A}^{-}$, comprising local donor (D) and acceptor (A) sites, the intervening bridge (B), and the surrounding medium (or solvent). In the two-state approximation (TSA), the kinetics may be modeled in terms of initial $\left(\psi_{\mathrm{i}}\right)$ and final $\left(\psi_{\mathrm{f}}\right)$ state wavefunctions, in which the transferring charge is localized primarily on the D and A sites, respectively.

Figure 2. Effective energy profiles along the reaction coordinate $(\mathrm{RC})$ for the initial and final diabatic states, indicating the reorganization energy $\left(E_{\mathrm{r}}\right)$, activation energy $\left(E_{a}\right)$, and reaction driving force $\left(-\Delta G_{0}\right)$. In a linear system, with parabolic profiles of equal curvature, the sum of the vertical energy gap at the equilibrium configuration for the initial state (DBA) and $-\Delta G_{0}$ is equal to $E_{r}[2,3]$.

Figure 3a. Schematic representation of optical and thermal et, corresponding, respectively, to the vertical transition with excitation energy hv and passage through the transition-state (or crossing) region.

Figure 3b. Sequence of photoinitiated electron transfer: charge separation (CS) from a locally excited state, followed by charge recombination (CR) back to the ground state The CS, CR notation is generally limited to cases where the D and A sites are initially charge neutral (as drawn).

Figure 4. [Figure 1 of [43], reprinted with permission. Copyright [1993] American Chemical Society]. Schematic depiction of NN superexchange coupling via two bridge units $\left(B_{1}\right.$ and $\left.B_{2}\right)$, each of which has an occupied ( $\left.\phi^{h}\right)$ and an unoccupied $\left(\phi^{e}\right)$ orbital. Orbital occupations relative to the defined reference (or"vacuum") state 
are indicated. The excitation energies of the virtual intermediate states relative to the degenerate initial $\left(\Psi_{D}^{0}\right)$ and final $\left(\psi_{A}^{0}\right)$ states are expressed in terms of the orbital energy differences defined in eq. 41 . The three primitive steps $(\rightarrow)$ in each "pathway" correspond to electron (e) or hole (h) transfer, or the creation (+eh) or destruction (-eh) of an electron/hole pair. Relative to the vacuum, the various states require the specification of at most three particles--the added electron and, in cases b-d, the eh pair. Pathway $c$ is obtained by interchanging the order of the $e$ and +eh steps in pathway $\mathrm{c}$, and pathway $\mathrm{d}^{\prime}$ is obtained by interchanging the e and -eh steps in pathway d. The set of six processes defines all pathways coupling $\psi_{D}^{0}$ and $\Psi_{A}^{0}$ in terms of $N N$ forward-directed (i.e., D to A) steps. In Scheme I of ref [43], also reprinted with permission, the minus sign preceding the " $\mathrm{e}_{1} \mathrm{~h}_{2}$ " contribution of $T_{\text {if }}$ (from pathways $c$ and $c^{\prime}$ ) is a typographical error and should be disregarded.

Figure 5. [Fig. 2 of [43], reprinted with permission. Copyright [1993] American Chemical Society]. Alternative representation of hole-transfer requiring the specification of only a single particle (h). See Figure 4 caption for definition of symbols.

Figure 6. $\pi$-type $\mathrm{D} / \mathrm{A}$ orbitals (the nonbonding orbitals of the terminal $\mathrm{CH}_{2}$ groups) linked by a trans-alkane bridge $\left(\left(\mathrm{CH}_{2}\right)_{\mathrm{m}+1}\right)$ possessing $\mathrm{m}$ covalent $\mathrm{CC}$ bonds (the covalent bonds connecting the $\mathrm{D}$ and $\mathrm{A}$ groups to the bridge contribute little to $\mathrm{T}_{\text {if }}$ $[34,43,64])$. The even and odd $m$ members correspond, respectively, to $C_{2 v}$ and $C_{2 h}$ point-group symmetry.

Figure 7. Plots of $\ln \left|T_{i f}\right|$ for $\left(\mathrm{CH}_{2}\right)_{m+3}$ radical cations and anions, $1 \pi(m)$, presented as a function of the number of bonds $(\mathrm{m})$ in the $\left(\mathrm{CH}_{2}\right)_{\mathrm{m}+1}$ bridge linking donor and acceptor $\mathrm{CH}_{2}$ groups (see fig 6). Decay coefficients $(\beta)$ for radical cation 
and anion systems are given at three levels, using a 3-21G orbital basis: energy splitting at the KT level (eq. (14)); direct SCF energy splitting (eq. (14)); and direct evaluation of $T_{\text {if }}$ (eq. (32)) using charge-localized SCF wavefunctions (see [43]). The $\beta$ values $\left(\AA^{-1}\right)$ are based on linear least-squares fits of $\ln \left|T_{\text {if }}\right|$ vs $m$, for $m=2-7$, with $\Delta r=1.27 \AA$ (see eq (11)).

Figure 8a. $\pi$-type $(2 \pi(m))$ and $\sigma$-type $(2 \sigma(m)) D / A$ orbitals linked by bicyclo [1.1.1]pentyl bridge units $(m=1-3)$, with adjacent units related by a staggered conformation. The odd and even m members correspond, respectively, to $\mathrm{C}_{2 \mathrm{v}}$ and $C_{2 h}$ point group symmetry for the $2 \pi(\mathrm{m})$ series, and $D_{3 d}$ and $D_{3 h}$ symmetry for $2 \sigma$ (m) series.

Figure $8 \mathrm{~b}$. Analogs of $2 \pi(2)$ and $2 \sigma(2)$, with bicyclo [1.1.1] pentane units replaced by biocyclo [2.2.2] octane units.

Figure 9. Coupling of $\mathrm{D} / \mathrm{A}$ orbitals (the nonbonding orbitals of the terminal $\mathrm{CH}_{2}$ groups) in a relative $\sigma$ orientation, mediated by a nonbonded sequence of $\mathrm{m} \mathrm{CH}_{4}$ spacer units. The odd- and even-m members correspond, respectively to $C_{2 v}$ and $\mathrm{C}_{2 \mathrm{~h}}$ point-group symmetry.

Figure 10. Schematic orbital diagram (roughly according to scale, based on calculated results), depicting the nearest-neighbor interaction of a pair of (a) bonding (CC) and (b) antibonding (CC*) NBO's [fig 5 of [49], reprinted with permission. Copyright [1992] American Chemical Society.]

Figure 11. Orbital diagrams indicating the most important pathways for electron transfer in radical anion systems. Results are obtained from perturbation analysis based on NBO's obtained at the KT/3-21G level. The orbital lobes are 
drawn approximately to scale on the basis of the calculated NBO's. The bonds (or antibonds) involved in each pathway are denoted by solid lines. Solid lines are also used to denote pathways symmetry-equivalent to those explicitly depicted by orbitals. Other bonds are either not shown ( $\mathrm{CH}$ bonds, except for those on the terminal $\mathrm{CH} 2$ groups in the case of $\pi$-transfer) or indicated by dashed lines. Each primitive step in a given pathway is denoted by an arrow [after fig 3 of [49], reprinted with permission. Copyright [1992] American Chemical Society.]

Figure 12. Adiabatic energy gaps for the lowest four states of the $\mathrm{Zn}_{2}\left(\mathrm{OH}_{2}\right)^{+}$ complex, based on state-averaged CASSCF calculations using a twelve orbital/three electron active space to allow correlation of the electrons in the full valence manifold of the $\mathrm{Zn}_{2}$ moiety, and with an augmented split-valence orbital basis for $\mathrm{Zn}$ and an SCF minimal basis for $\mathrm{H}_{2} \mathrm{O}$ [77]. The lower and higher energy pairs of states at each of the three $\mathrm{ZnO}$ distances correspond roughly to the respective valence electronic configurations $\left(4 s, 4 s^{\prime}\right)^{3}$ and $(4 s, 4 s)^{2}\left(4 \rho \sigma, 4 \rho \sigma^{\prime}\right)^{1}$, where the prime denotes the $\mathrm{Zn}$ atom nearest the water molecule.

Figure 13. DBA systems 8 and 9 , involving anthracene donor and substituted olefin acceptor groups linked, respectively, by double relays of 7 and $9 \mathrm{CC}$ bonds [104]. Space-filling profiles are depicted by CPK structures. 


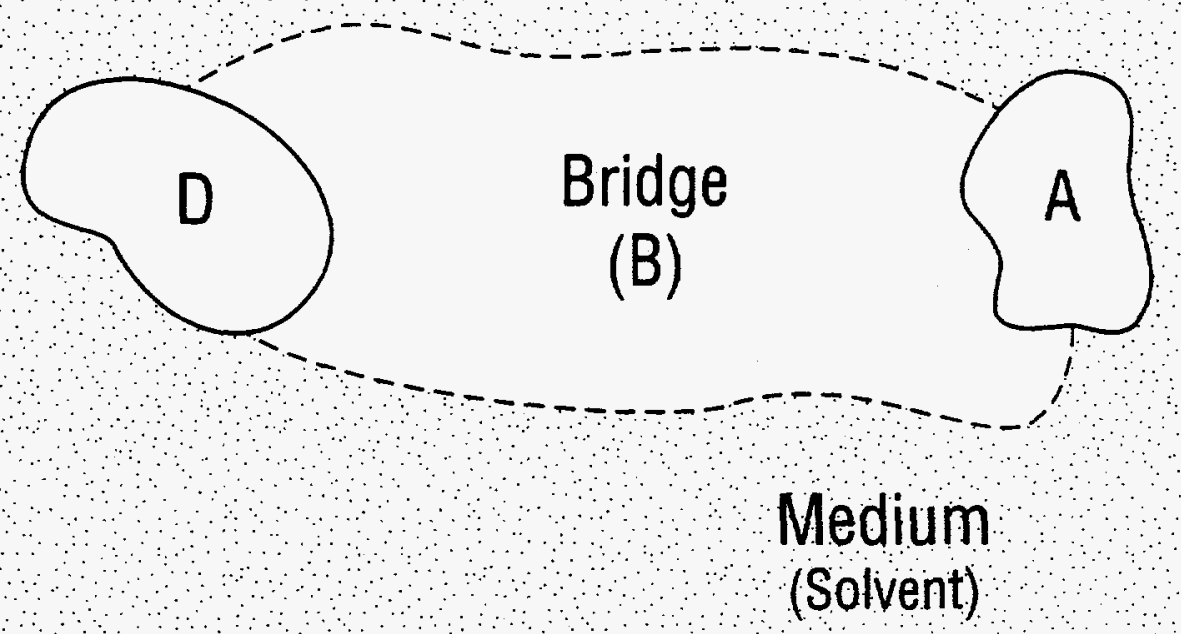

$\begin{array}{llll}\psi_{i} & \Leftrightarrow & \{\mathrm{DBA}\} & \text { (initial state) } \\ \psi_{\mathrm{f}} & \Leftrightarrow\left\{\mathrm{D}^{+} \mathrm{BA}^{-}\right\} & \text {(final state) }\end{array}$

Fig. 1 


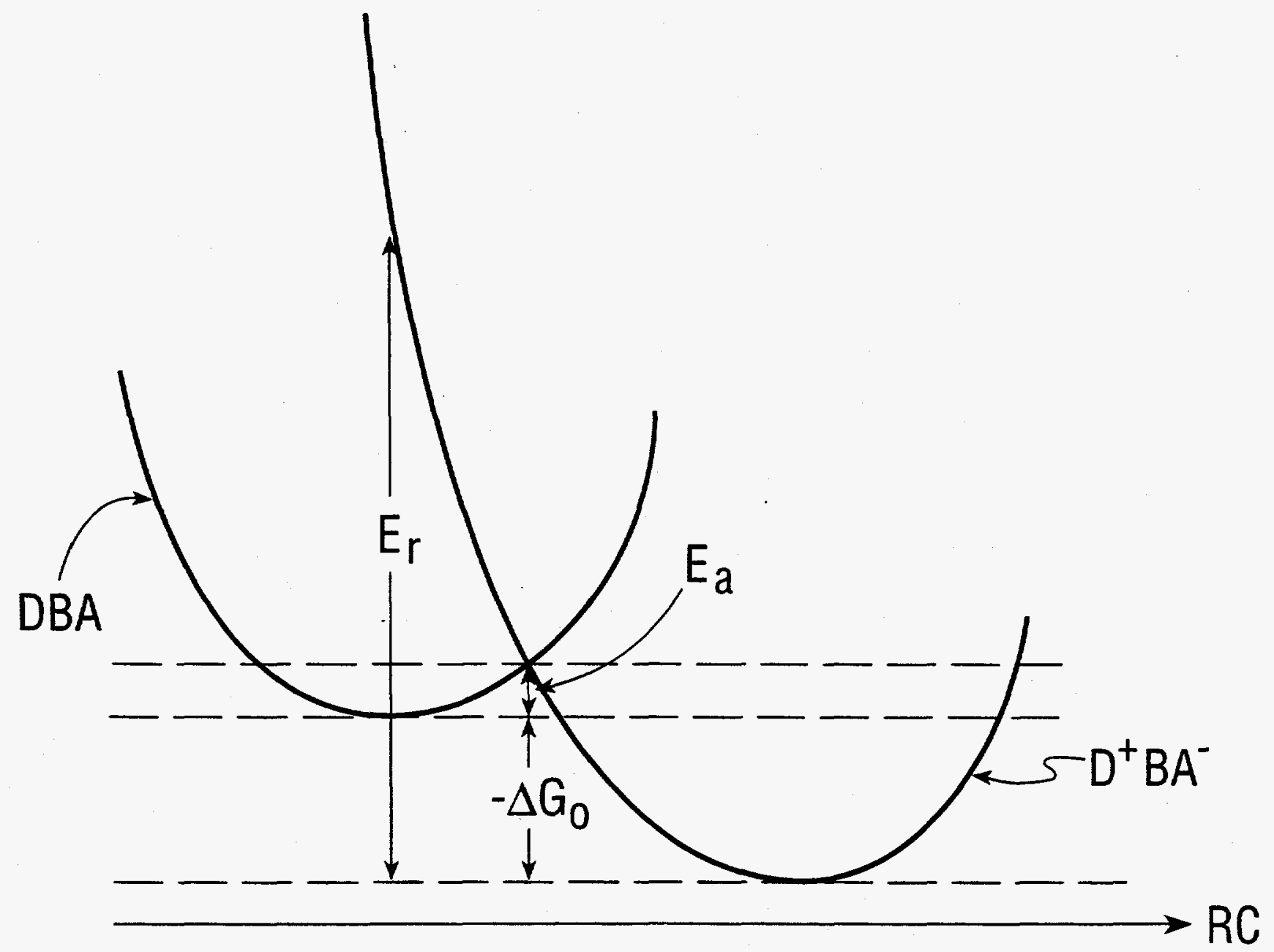

Fig. 2 
(a)

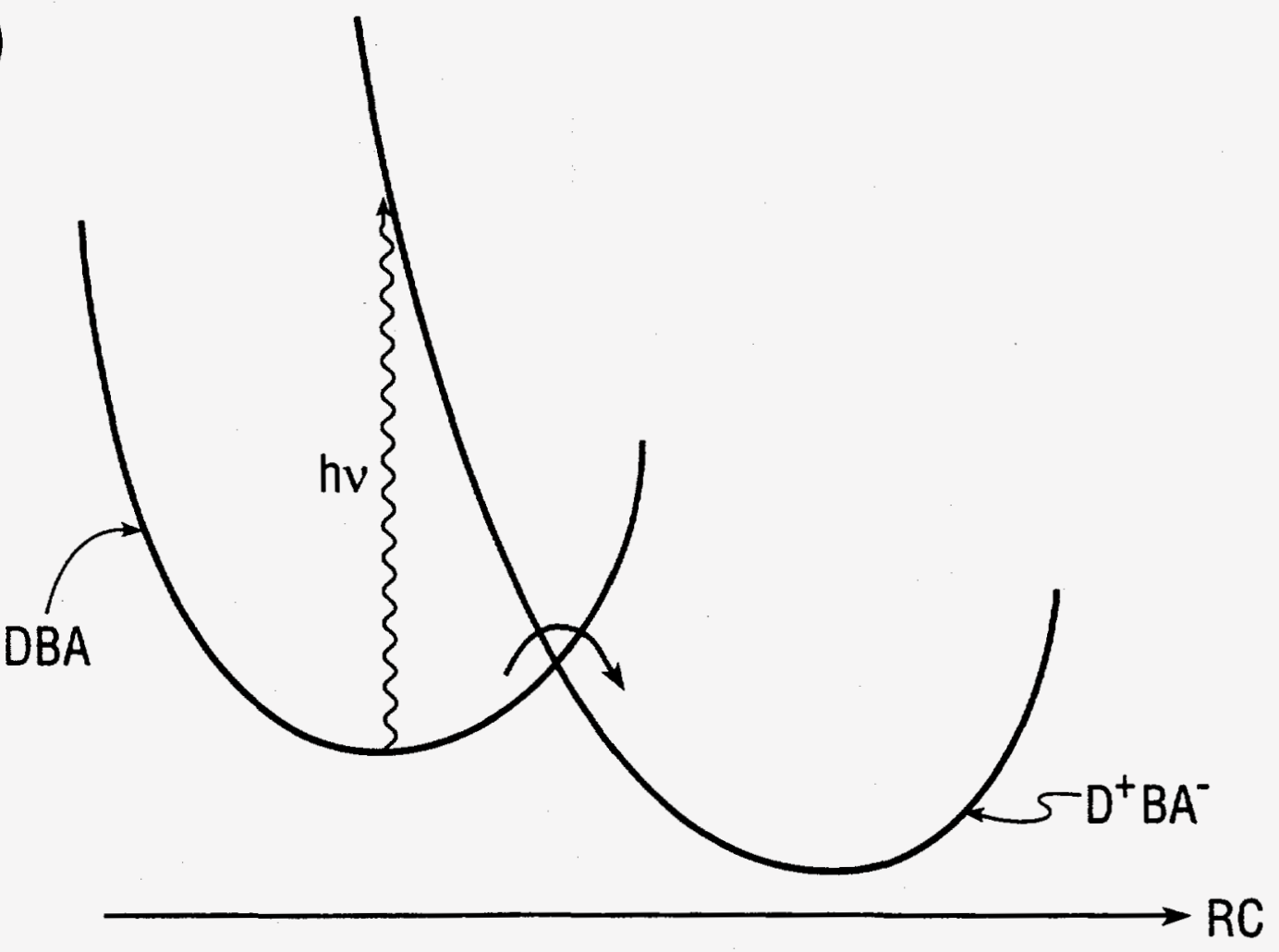

(b)

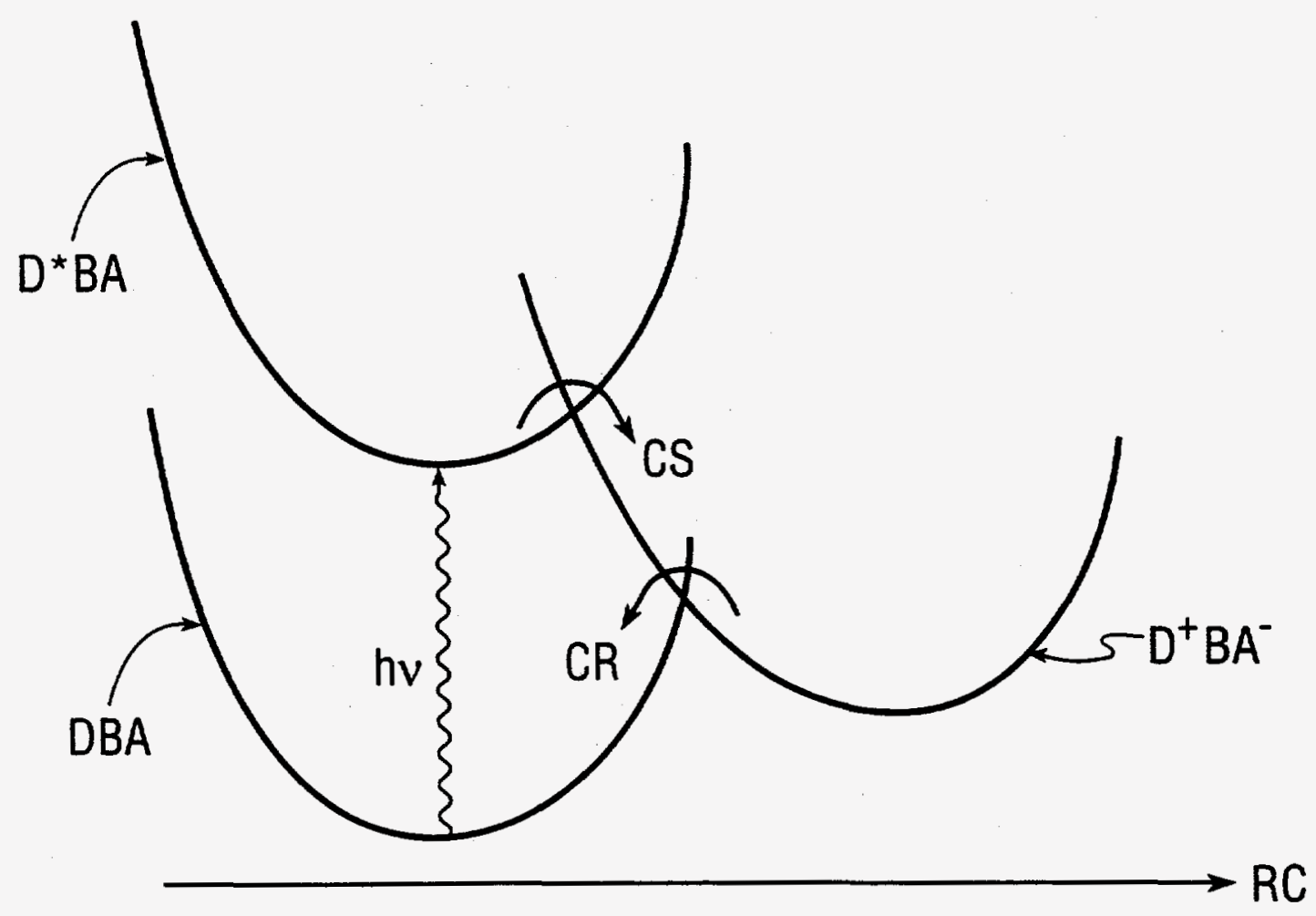

Fig. 3 


\section{Superexchange D/A Coupling via Two Spacer \\ Groups, $B_{1}$ and $B_{2}$}

Reference ("vacuum") State:

$\mathrm{e} \equiv$ electron
$h \equiv$ hole

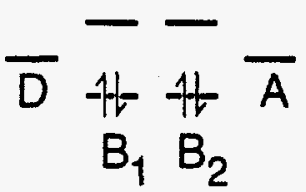

(a) e-transfer (1-particle)

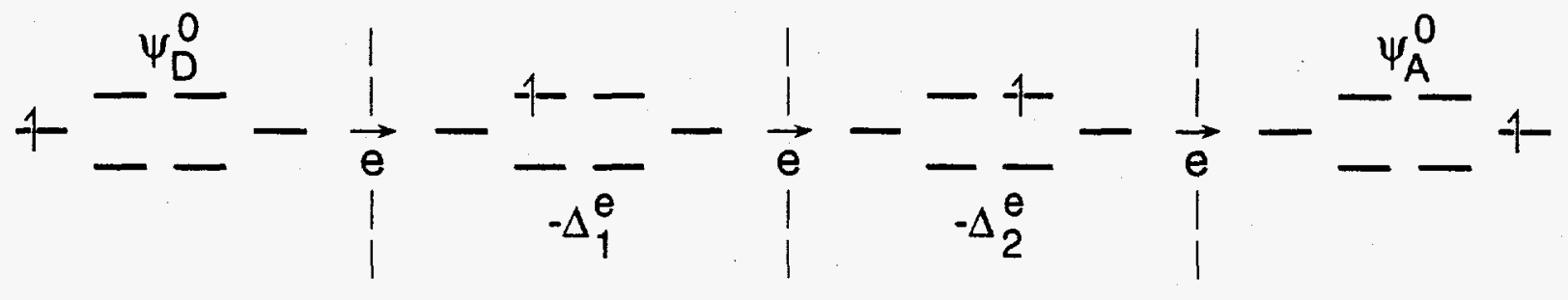

(b) h-transfer (3-particle)

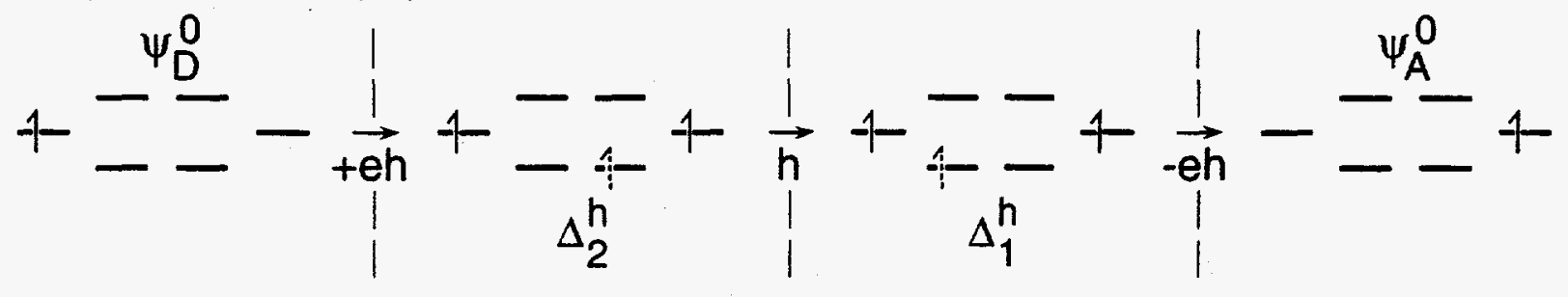

(c) hybrid $\left(e_{1} / h_{2}\right)$ transfer (3-particle)

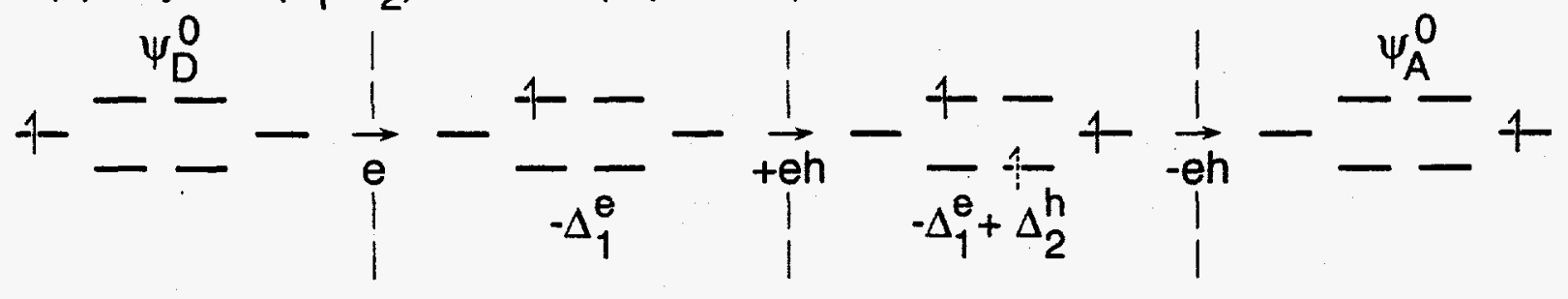

(d) hybrid $\left(h_{1} / e_{2}\right)$ transfer (3-particle)

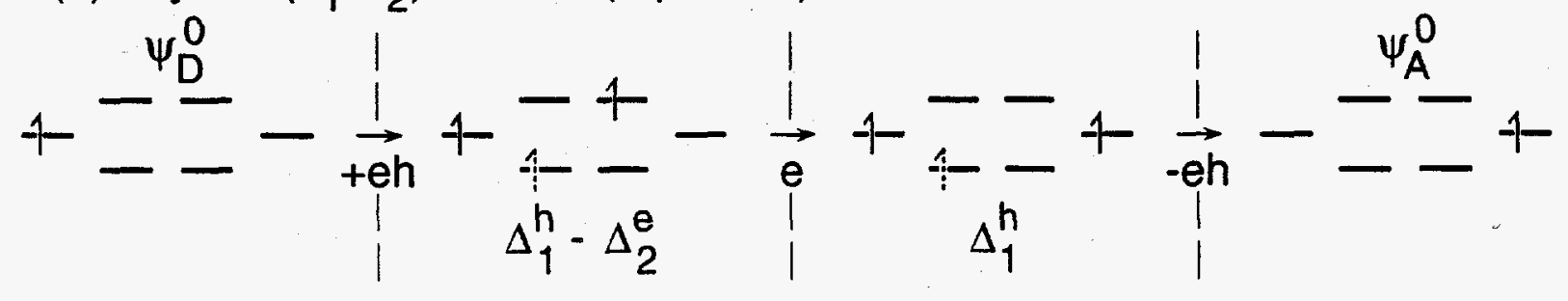

Fig. 4 


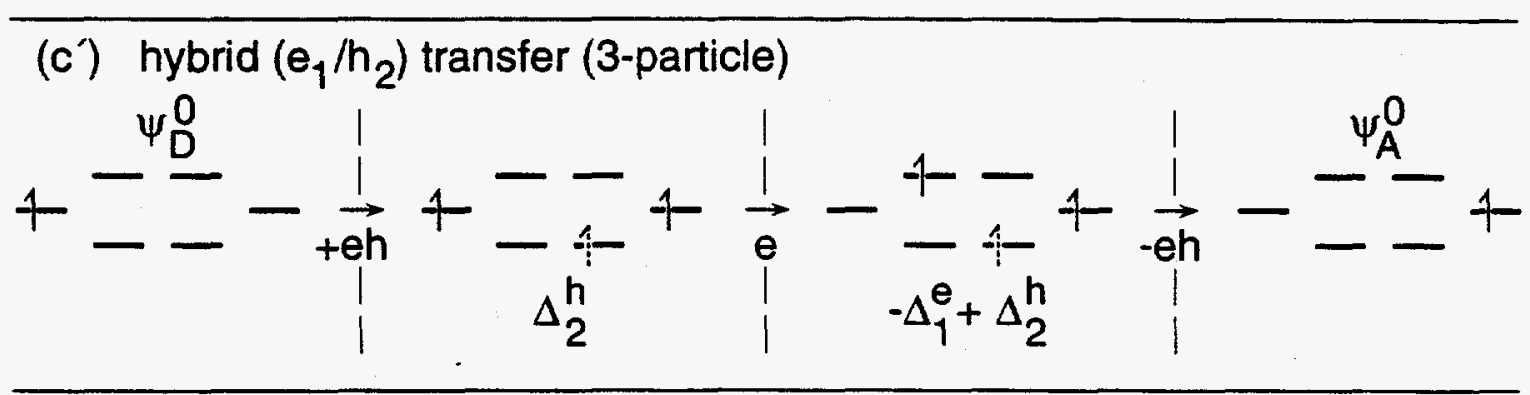

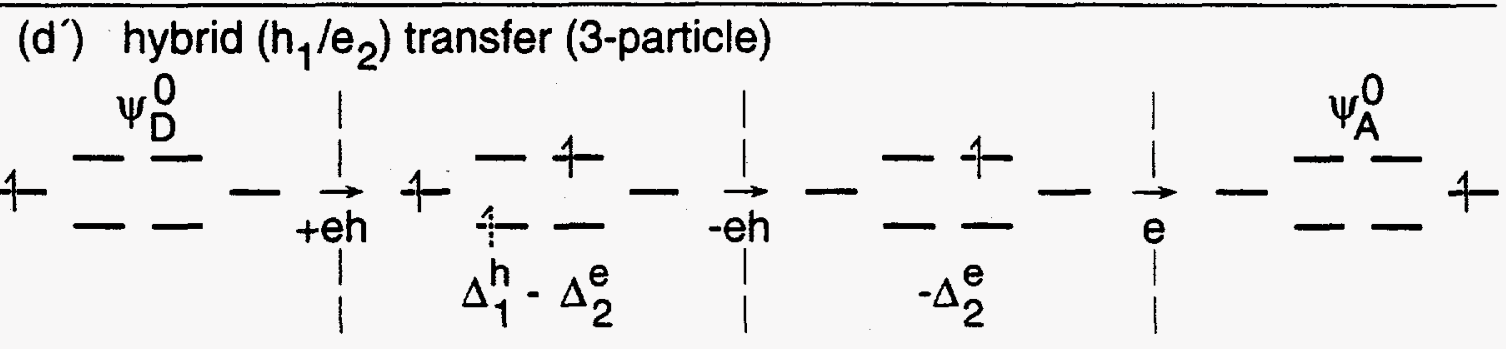

Fig. 4 (cont.) 


\section{Alternative Representation of $\mathbf{h}$-transfer}

Reference ("vacuum") State:

$$
\begin{array}{ccc}
\mathbb{A} & - & -1- \\
D & \mathbb{H} & A \\
B_{1} & B_{2}
\end{array}
$$

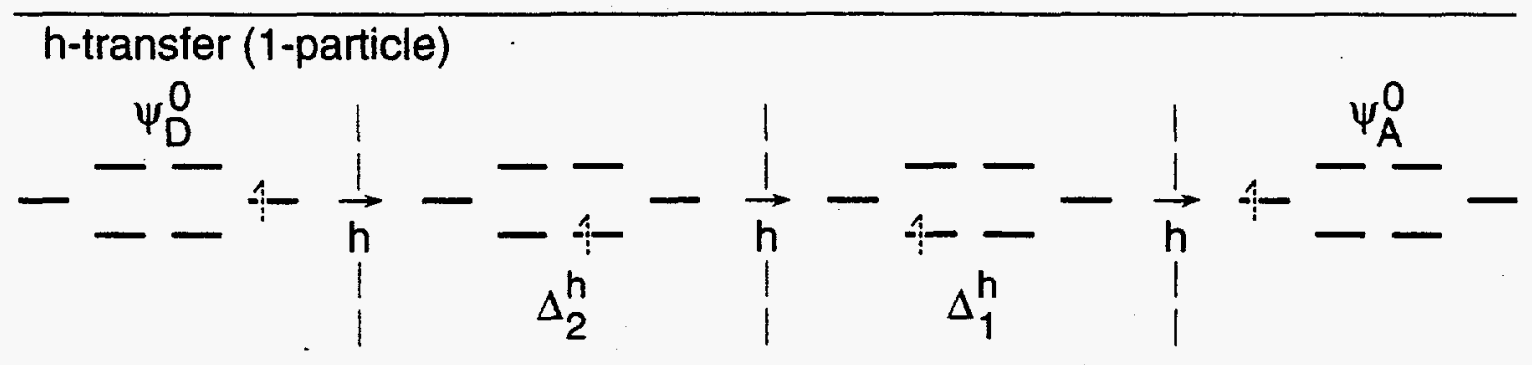

Fig. 5 

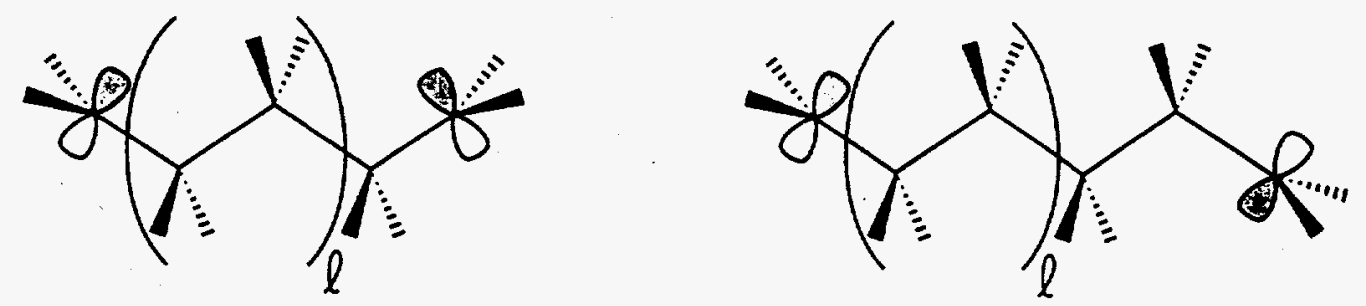

$$
C_{2 v} \quad m=2 \ell, \ell \geq 1
$$

$$
C_{2 h} \quad m=2 \ell+1, \ell \geq 0
$$

$1 \pi(m)$

Fig. 6 
$\left(\mathrm{CH}_{2}\right)_{m+3}^{+}$D/A coupling

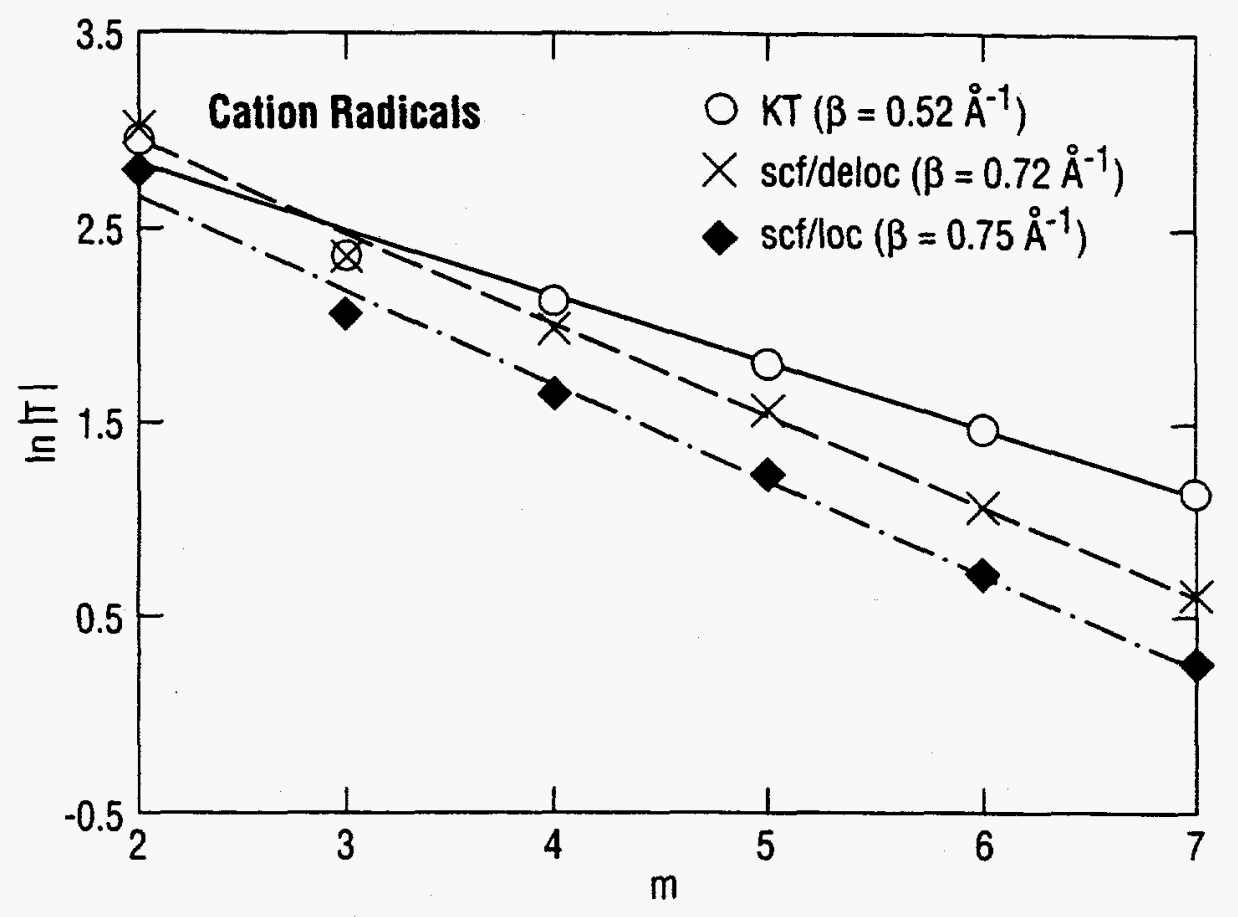

$\left(\mathrm{CH}_{2}\right)_{m+3}^{-}$D/A coupling

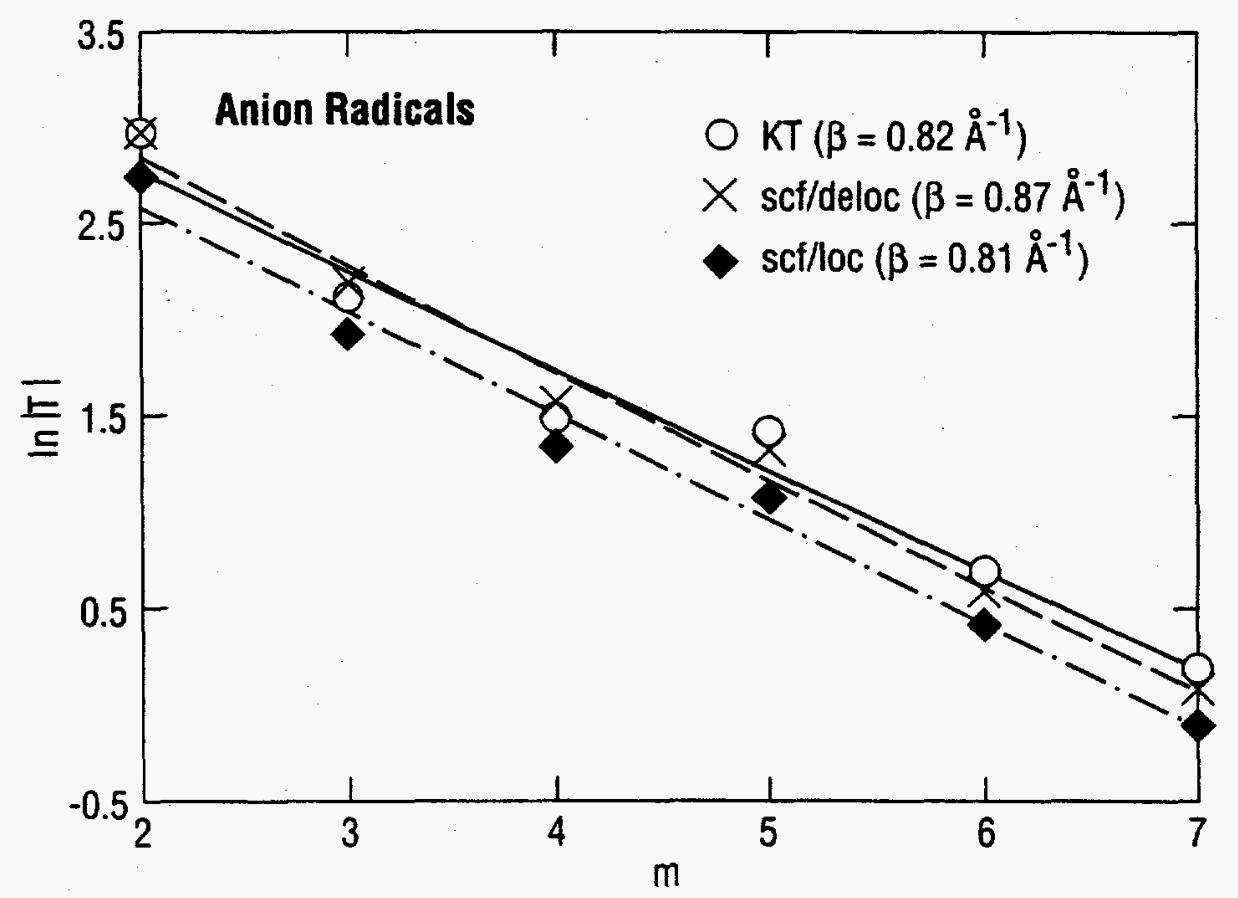

Fig. 7 
(a) bicyclo [1.1.1] pentane spacers
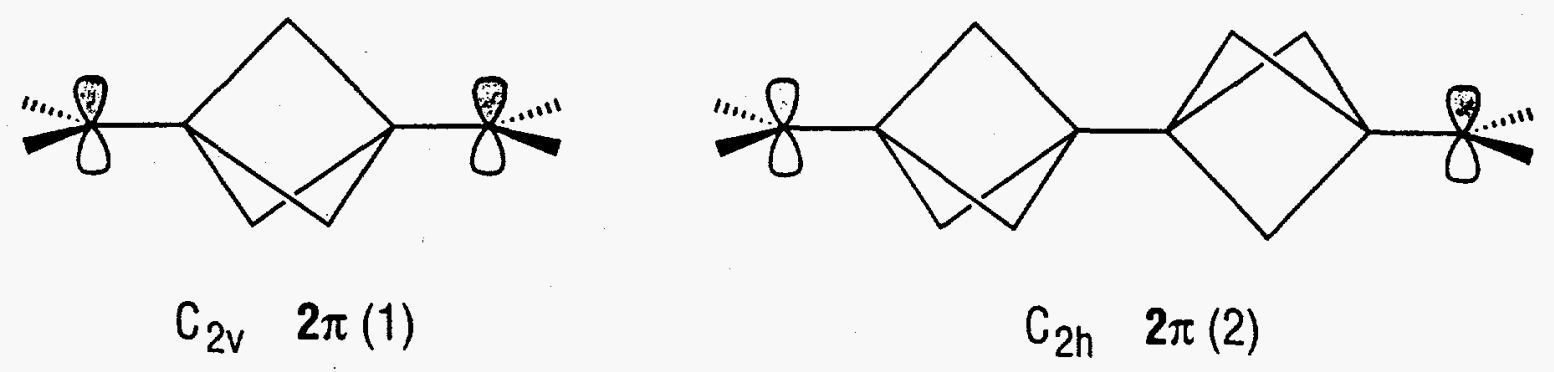

$$
C_{2 v} 2 \pi(1) \quad C_{2 h} 2 \pi(2)
$$

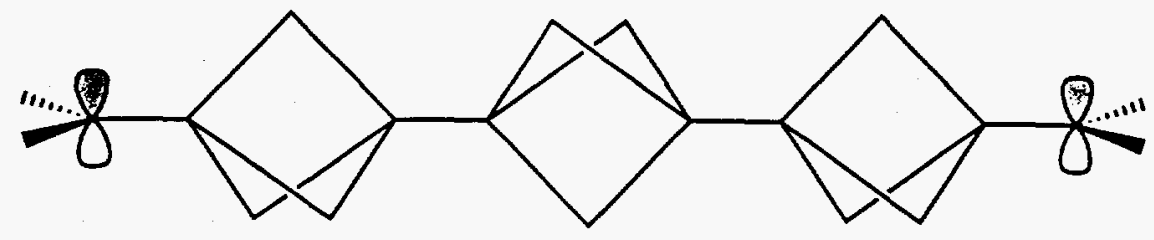

$C_{2 v} \quad 2 \pi(3)$
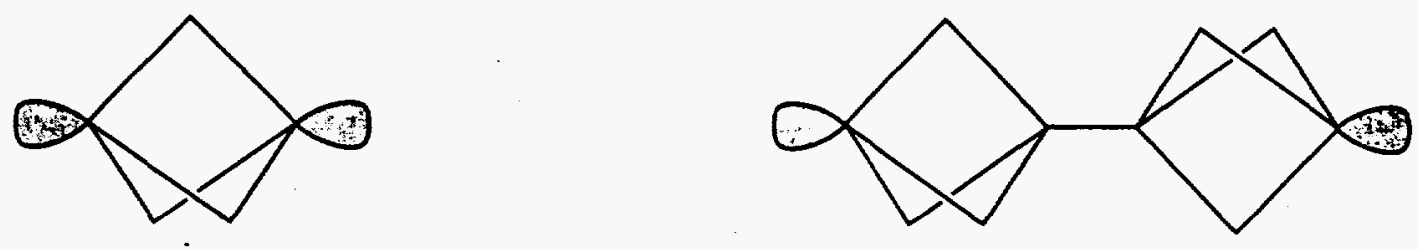

$D_{3 h} \quad 2 \sigma(1)$

$D_{3 d} \quad 2 \sigma(2)$

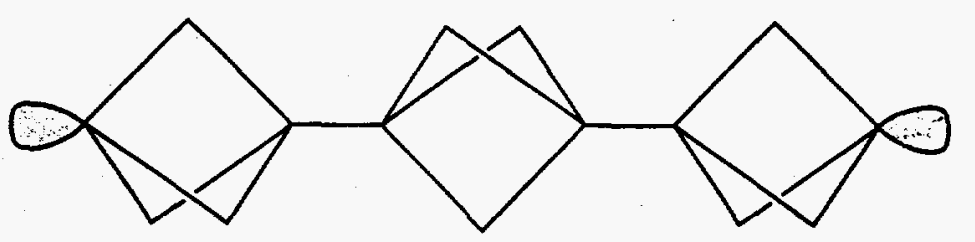

$D_{3 h} \quad 2 \sigma(3)$

Fig. 8 
(b) bicyclo [2.2.2] octane spacers
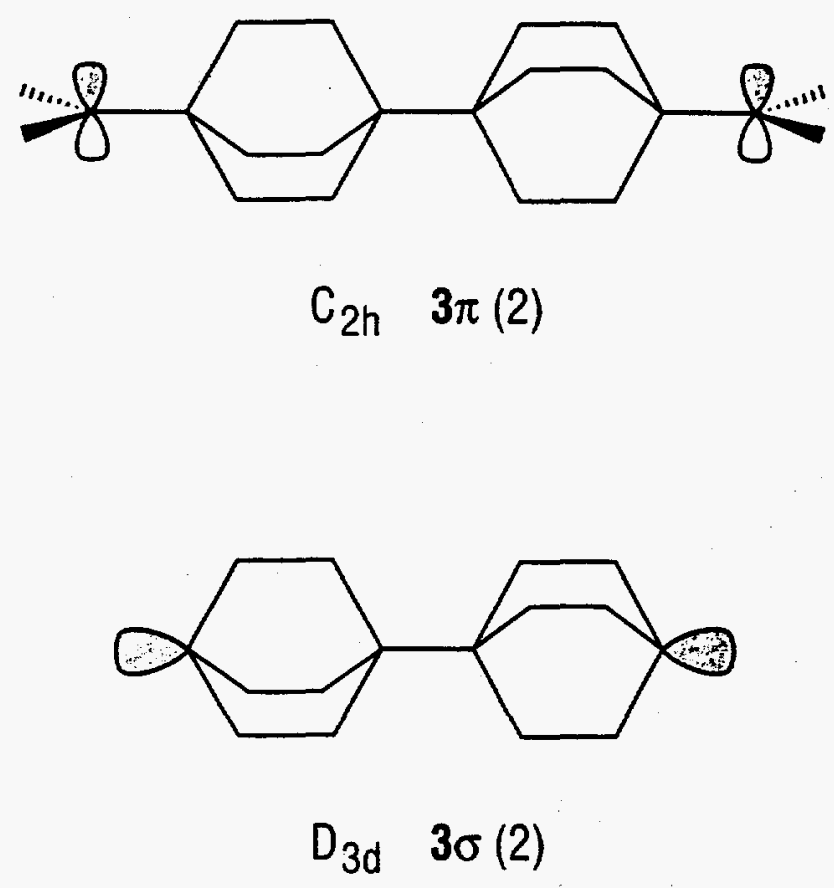

Fig. $8 b$ 
do
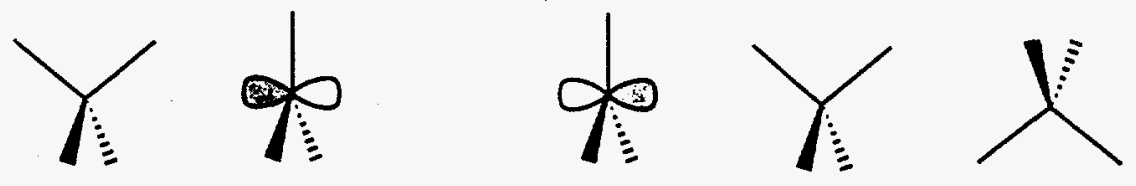

$\infty$

$C_{2 v} \quad 4 \sigma(1)$

$C_{2 h} \quad 4 \sigma(2)$

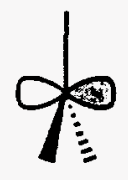
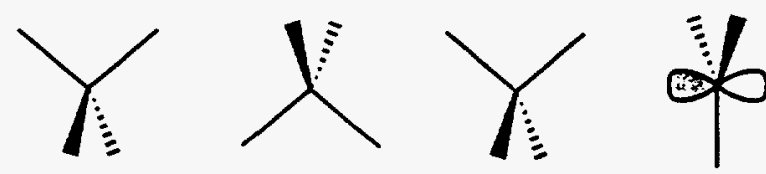

$C_{2 v} \quad 4 \sigma(3)$

Fig. 9 
(a)

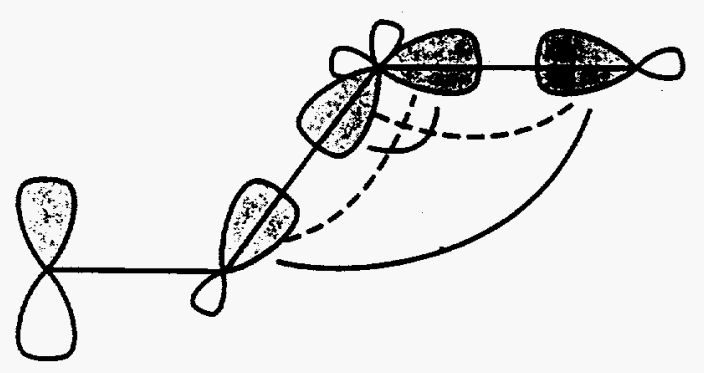

(b)

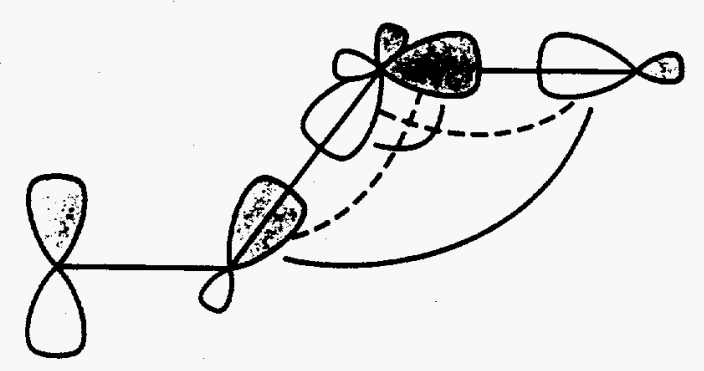

Fig. 10 
Radical Anions
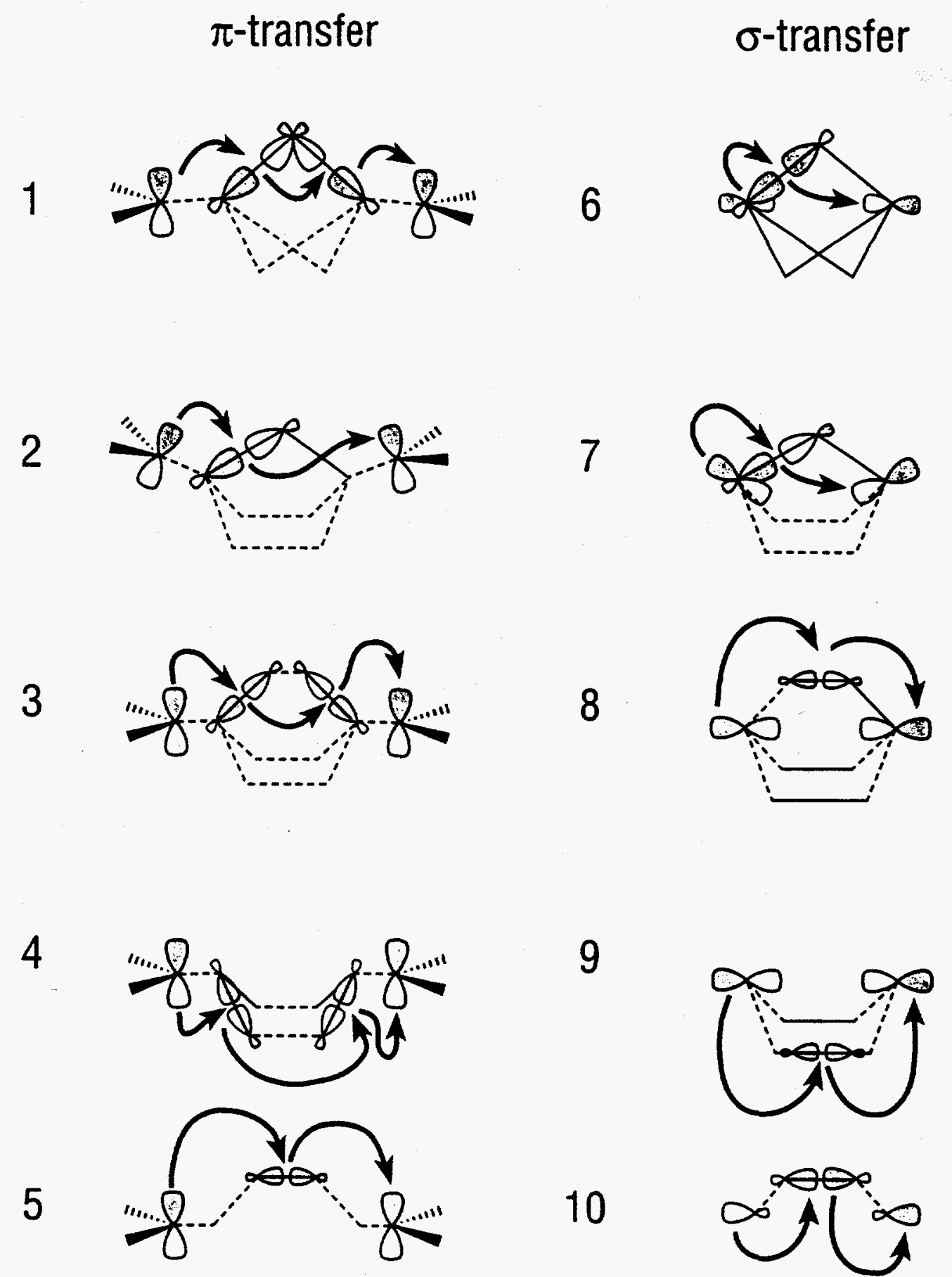


\section{Lowest States of $\left(\mathrm{Zn}_{2} \mathrm{OH}_{2}\right)^{+}$ \\ (3 electrons in $4 s / 4 p \sigma / 4 s^{\circ} / 4 p \sigma^{\circ}$ manifold)}

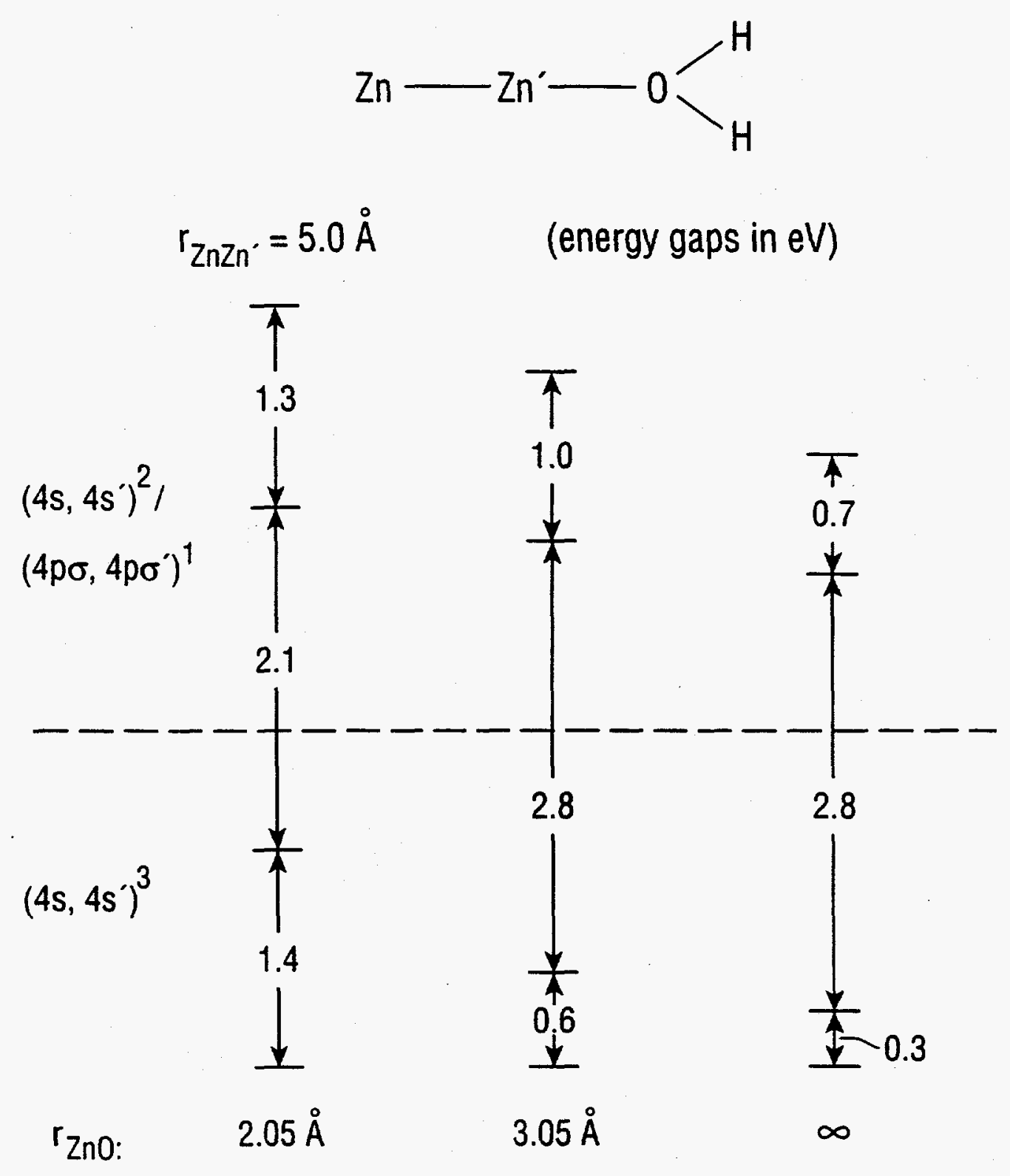

Fig. 12 


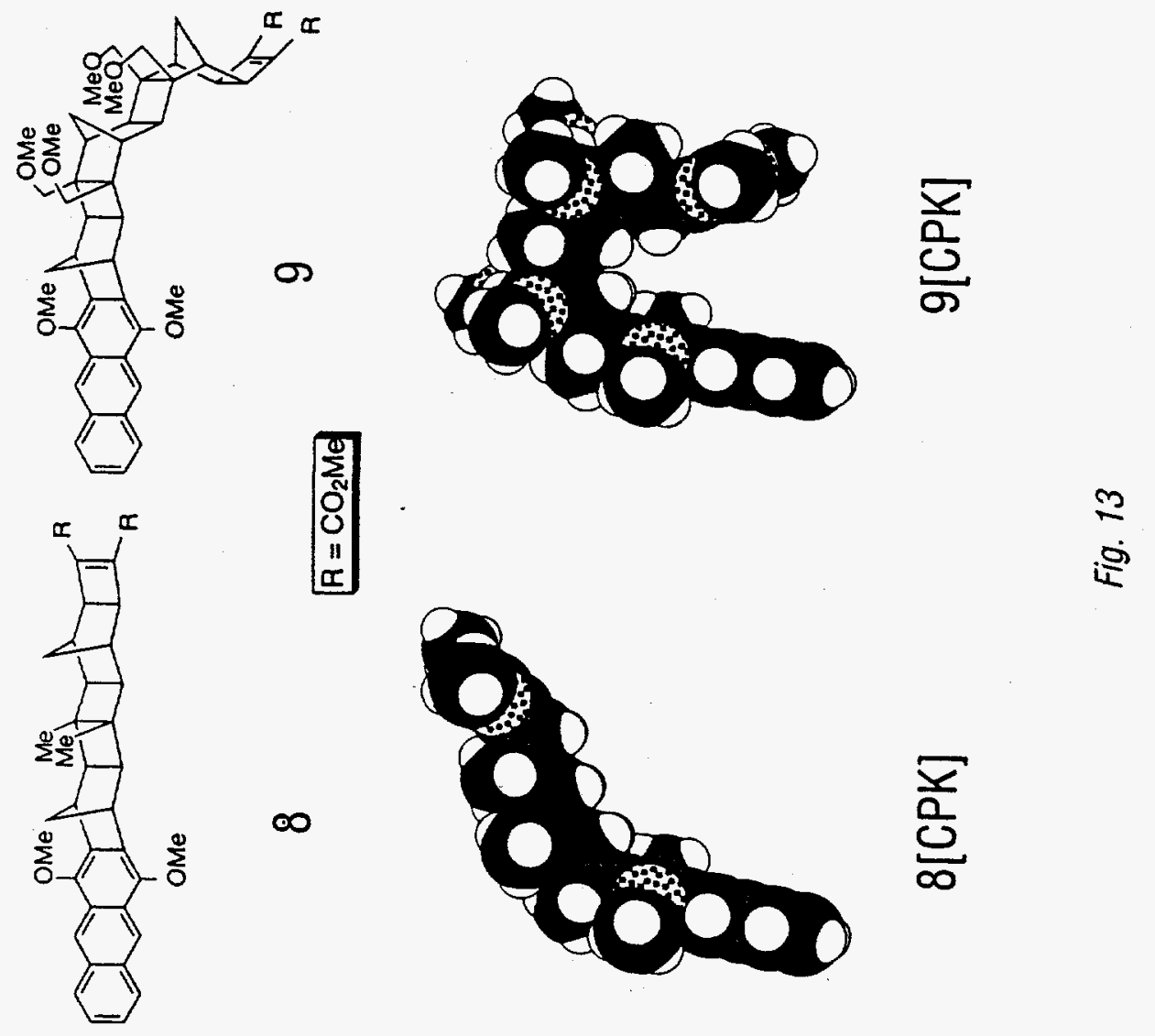




\section{Structures}
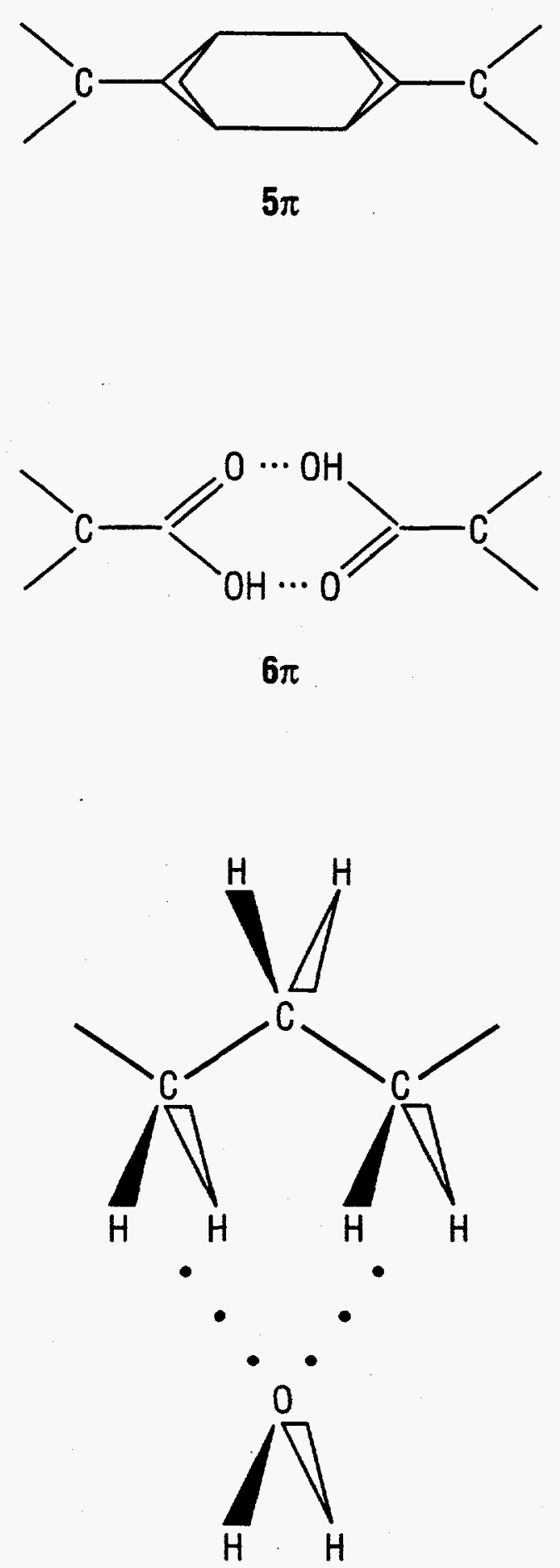

$7 \pi$ 


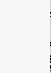

